\title{
WestVirginiaUniversity
}

THE RESEARCH REPOSITORY @ WVU

Graduate Theses, Dissertations, and Problem Reports

2005

\section{Maximum size t-cross-intersecting and intersecting families with degree conditions}

\author{
Yongbin Ou \\ West Virginia University
}

Follow this and additional works at: https://researchrepository.wvu.edu/etd

\section{Recommended Citation}

Ou, Yongbin, "Maximum size t-cross-intersecting and intersecting families with degree conditions" (2005). Graduate Theses, Dissertations, and Problem Reports. 2263.

https://researchrepository.wvu.edu/etd/2263

This Dissertation is protected by copyright and/or related rights. It has been brought to you by the The Research Repository @ WVU with permission from the rights-holder(s). You are free to use this Dissertation in any way that is permitted by the copyright and related rights legislation that applies to your use. For other uses you must obtain permission from the rights-holder(s) directly, unless additional rights are indicated by a Creative Commons license in the record and/ or on the work itself. This Dissertation has been accepted for inclusion in WVU Graduate Theses, Dissertations, and Problem Reports collection by an authorized administrator of The Research Repository @ WVU.

For more information, please contact researchrepository@mail.wvu.edu. 


\title{
Maximum size $t$-cross-intersecting and intersecting families with degree conditions
}

\author{
Yongbin $\mathrm{Ou}$ \\ Dissertation submitted to the \\ Eberly College of Arts and Sciences \\ at West Virginia University \\ in partial fulfillment of the requirements \\ for the degree of \\ Doctor of Philosophy \\ in \\ Mathematics \\ John L. Goldwasser, Ph.D., Chair \\ Elaine M. Eschen, Ph.D. \\ Hong-Jian Lai, Ph.D. \\ Cun-Quan Zhang, Ph.D. \\ Jerzy Wojciechowski, Ph.D. \\ Department of Mathematics \\ West Virginia University \\ 2005
}

Keywords: extremal set theory, intersecting, shadow, Sperner 


\begin{abstract}
Maximum size $t$-cross-intersecting and intersecting families with degree conditions
\end{abstract}

\title{
Yongbin $\mathrm{Ou}$
}

We present four main results: (1) A solution to the problem of finding two set systems $\mathcal{A}$ and $\mathcal{B}$ such that $\mathcal{A}$ is $r_{1}$-intersecting, $\mathcal{B}$ is $r_{2}$-intersecting, $\mathcal{A}, \mathcal{B}$ are $t$-cross-intersecting and $|\mathcal{A}|+|\mathcal{B}|$ is a maximum; (2) A solution to the problem of finding two set systems $\mathcal{A}$ and $\mathcal{B}$ such that $\mathcal{A}, \mathcal{B}$ are Sperner, $t$-cross-intersecting and $|\mathcal{A}|+|\mathcal{B}|$ is a maximum; (3) A solution to the problem of finding the maximum size of an intersecting set system $\mathcal{F}$ such that the complementary degree $c(\mathcal{F})=s$ for a specified value $s$; (4) An asymptotic result on the complementary degree of an intersecting set system. 


\section{Acknowledgments}

I would like to thank my advisor, Dr. John Goldwasser, for his guidance and help on my study, research and this dissertation. Without him, this dissertation could not have been completed.

I would also like to thank other committee members: Dr. Hong-Jian Lai , Dr. Elaine Eschen, Dr. Cun-Quan Zhang and Dr. Jerzy Wojciechowski for their help during my studies. 


\section{DEDICATION}

To

my father, my mother 


\section{Contents}

1 Introduction 1

2 Intersecting and $t$-cross-intersecting Set Systems 5

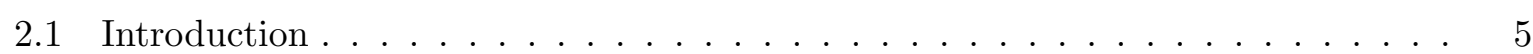

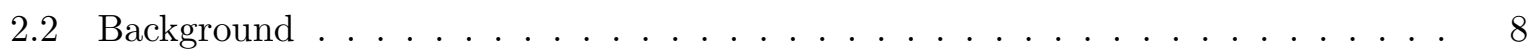

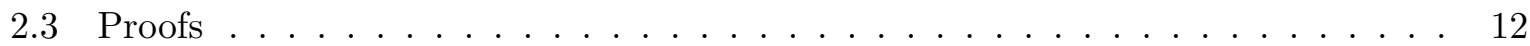

3 Sperner $t$-cross-intersecting Set Systems 19

3.1 Introduction . . . . . . . . . . . . . . . . . . . . . . 19

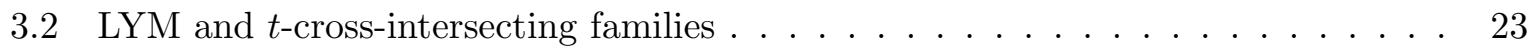

3.3 Proof of Theorem $3.1 .5 \ldots \ldots \ldots \ldots \ldots \ldots \ldots \ldots$

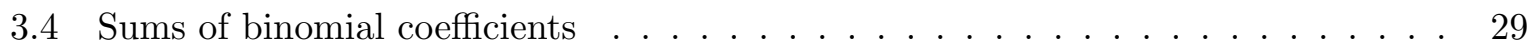

4 Intersecting Set Systems with Degree Conditions 33

4.1 Notation and Background . . . . . . . . . . . . . . . . . . . . . 33

4.2 Shadows of two set systems $\ldots \ldots \ldots \ldots \ldots \ldots$ 
4.3 Minimum shadow with degree conditions . . . . . . . . . . . . . . . . . 41

4.4 Intersecting set systems with degree conditions $\ldots \ldots \ldots \ldots$

5 An Asymptotic Bound and Non-existence Theorem $\quad 71$

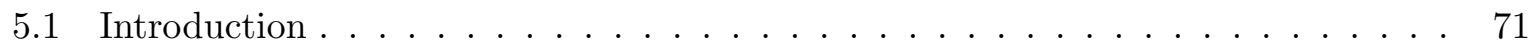

5.2 Intersecting set systems with a fractional degree condition . . . . . . . . . . 71

5.3 Minimum complementary degree of an intersecting set system . . . . . . . . . 74 


\section{Chapter 1}

\section{Introduction}

Let $X=\{1,2, \cdots, n\}, X^{(k)}=\{F \subseteq X:|F|=k\}$ and let $\mathcal{F}$ be a subset of the power set $\mathcal{P}(X)$ of $X$. Finding the maximum or minimum size of $\mathcal{F}$ where conditions are imposed on the intersection and/or containment properties of sets in $\mathcal{F}$ is at the core of extremal set theory. The Erdős-Ko-Rado theorem, the Kruskal-Katona theorem, and Sperner's theorem are all concerned with variations of this problem. The results in this dissertation are applications, generalizations, and extensions of these three fundamental theorems.

A set system $\mathcal{F} \subseteq \mathcal{P}(X)$ is intersecting if $A \cap B \neq \emptyset$ for every $A, B \in \mathcal{F}$. The Erdős-Ko-Rado theorem gives the maximum size of an intersecting set system.

Theorem. (Erdős-Ko-Rado Theorem[3]) If $2 k \leq n$ and $\mathcal{F} \subseteq X^{(k)}$ is intersecting then $|\mathcal{F}| \leq$ $\left(\begin{array}{l}n-1 \\ k-1\end{array}\right)$.

When $2 k<n$ equality is achieved only by the so-called trivial set system, which is the collection of all $k$-subsets of $X$ containing a fixed element.

The intersecting concept can be generalized to $t$-intersecting. $\mathcal{F}$ is $t$-intersecting if $|A \cap B| \geq t$ for every $A, B \in \mathcal{F}$. Another way to generalize intersecting is to look at two set systems $\mathcal{A}$ and $\mathcal{B}$. $\mathcal{A}$ and $\mathcal{B}$ are $t$-cross-intersecting if $|A \cap B| \geq t$ for every $A \in \mathcal{A}$ and $B \in \mathcal{B}$.

If $n+r$ is even we let $\mathcal{F}(n, r)=\left\{F \subseteq X:|F| \geq \frac{n+r}{2}\right\}$ (while if $n+r$ is odd we let $\mathcal{F}(n, r)$ be equal to $\mathcal{F}(n, r+1)$ plus a few more sets, defined precisely in Chapter 2). Clearly $\mathcal{F}(n, r)$ is $r$-intersecting. Katona showed that if $\mathcal{A} \subseteq \mathcal{P}(X)$ is $r$-intersecting then $|\mathcal{A}| \leq|\mathcal{F}(n, r)|$. Frankl conjectured that if $\mathcal{A}, \mathcal{B} \subseteq \mathcal{P}(X)$ are $t$-cross-intersecting with $|\mathcal{A}| \geq|\mathcal{B}|$ then $|\mathcal{B}| \leq|\mathcal{F}(n, t)|$. 
In Chapter 2 we prove this conjecture is correct. The main result is Theorem 2.1.8 in which we characterize all $\mathcal{A}, \mathcal{B} \subseteq \mathcal{P}(X)$ such that $\mathcal{A}$ is $r_{1}$-intersecting, $\mathcal{B}$ is $r_{2}$-intersecting, $\mathcal{A}, \mathcal{B}$ are $t$-cross-intersecting and $|\mathcal{A}|+|\mathcal{B}|$ is a maximum. The proof of Theorem 2.1.8 uses Harper's theorem on the Hamming distance between subsets of $\mathcal{P}(X)$ and theorems of Frankl, Hilton, and Katona on intersecting and cross-intersecting families, which are all based on the KruskalKatona theorem on minimum shadow. In fact many proofs of intersection theorems make use of minimum shadow theorems.

For $\mathcal{F} \subseteq X^{(k)}$, the shadow of $\mathcal{F}$ is

$$
\partial \mathcal{F}=\{A:|A|=k-1 \text { and } A \subseteq F \text { for some } F \in \mathcal{F}\}
$$

The Kruskal-Katona theorem states that to choose $m$ sets in $X^{(k)}$ such that the size of the shadow is minimized, we pick the first $m$ sets of $X^{(k)}$ in the so-called COLEX order. The definition of the COLEX order will be given in Chapter 2 .

A set system $\mathcal{F} \subseteq \mathcal{P}(X)$ is Sperner if $A \nsubseteq B$ and $B \nsubseteq A$ for every $A, B \in \mathcal{F}$. Milner found the maximum size of a $t$-intersecting Sperner family of sets and Frankl proved a generalization to $t$-cross-intersecting Sperner families.

In Chapter 3 we prove theorems that generalize both of these results. Let $\mathcal{A}_{1}, \mathcal{A}_{2}, \cdots, \mathcal{A}_{q}$ be Sperner families of subsets of $X$ which are pairwise $t$-cross-intersecting. We determine the maximum values of $\sum\left|\mathcal{A}_{i}\right|$ and $\prod\left|\mathcal{A}_{i}\right|$ and find all optimal configurations.

We give two proofs for one of the main results (Theorem 3.1.4) of Chapter 3. One uses the standard technique of modifying a Sperner set system by replacing a collection of sets by its shadow, with the Kruskal-Katona theorem providing a bound on the relative sizes of these two collections of sets. The other uses the LYM inequality (a generalization of Sperner's theorem) and convexity in an unusual way.

If $\mathcal{F} \subseteq \mathcal{P}(X)$ is a family of sets and $i \in X$, the degree $d_{\mathcal{F}}(i)$ of $i$ in $\mathcal{F}$ is the number of sets in $\mathcal{F}$ containing $i$, the maximum degree $d(\mathcal{F})$ of $\mathcal{F}$ is the maximum over all $i$ in $X$ of $d_{\mathcal{F}}(i)$, and $c(\mathcal{F})=|\mathcal{F}|-d(\mathcal{F})$ is the minimum complementary degree of $\mathcal{F}$. The optimal configuration in the Erdös-Ko-Rado theorem, the so-called trivial set system, has $d(\mathcal{F})=|\mathcal{F}|($ and $c(\mathcal{F})=0$ ). The Hilton-Milner theorem solves the problem of finding the maximum size of an intersecting set system $\mathcal{F} \subseteq X^{(k)}$ such that $c(\mathcal{F})>0$. Frankl and Goldwasser proved generalizations of the Hilton-Milner theorem for intersecting set systems $\mathcal{F} \subseteq X^{(k)}$ with $c(\mathcal{F}) \leq s$ for $s \leq\left(\begin{array}{c}n-3 \\ k-2\end{array}\right)$. This upper bound for $s$ is significant because with this restriction the optimal configurations have a particularly simple structure, which is lost for large value of $s$. In Chapter 4 we find

the largest intersecting family $\mathcal{F} \subseteq X^{(k)}$ such that $c(\mathcal{F})=s$ where $s \in\left(\left(\begin{array}{c}n-3 \\ k-2\end{array}\right), 4\left(\begin{array}{c}n-5 \\ k-3\end{array}\right)+\left(\begin{array}{c}n-5 \\ k-4\end{array}\right)\right]$. The endpoints of this interval of values of $s$ are determined by two intersecting families with 
a particularly simple structure. The proof of optimality for this range of values of $s$ is quite long and complicated. A key result needed in this proof is Theorem 4.2.1, a generalization of the Kruskal-Katona theorem (which is likely to be useful in other contexts) which solves the following problem:

Problem. Let $\mathcal{A}, \mathcal{B} \subseteq X^{(k)},|\mathcal{A}|+|\mathcal{B}|=r,|\mathcal{A}| \geq|\mathcal{B}| \geq t, 2 t<r$. Find the minimum of $|\partial \mathcal{A}|+|\partial \mathcal{B}|$.

The special case $t=0$ is the Kruskal-Katona theorem.

Another way to impose a degree condition on an intersecting family $\mathcal{F} \subseteq X^{(k)}$ is to require $d(\mathcal{F}) \leq e|\mathcal{F}|$ for some real number $e \in(0,1)$. Let $\mathcal{F}_{(3,2)}=\left\{F \in X^{(k)}:|F \cap\{1,2,3\}| \geq\right.$ $2\}$ and $\mathcal{F}_{(3,2)}^{*}=\left\{F \in X^{(k)}:|F \cap\{1,2,3\}|=2\right\}$. In a well known but unpublished paper, Erdős, Rothschild and Szemerédi showed that if $e=\frac{2}{3}$ then $|\mathcal{F}| \leq\left|\mathcal{F}_{(3,2)}\right|$ for fixed $k$ and $n$ sufficiently large. With the additional assumption that $\mathcal{F}$ is maximal, Frankl showed the same conclusion holds for any $e \in\left(\frac{2}{3}, 1\right)$ and that equality holds for $e$ in this range if and only if $\mathcal{F}$ is isomorphic to $\mathcal{F}_{(3,2)}$. Of course equality can never hold if $e=\frac{2}{3}$ because $d\left(\mathcal{F}_{(3,2)}\right)>\frac{2}{3}|\mathcal{F}|$. In Chapter 5 we sharpen the theorem of Erdős, Rothschild and Szemerédi by proving a $t$ intersecting generalization where the special case $t=1$ says that for fixed $k$ if $d(\mathcal{F}) \leq \frac{2}{3}|\mathcal{F}|$ and $n$ is sufficiently large, then $|\mathcal{F}| \leq\left|\mathcal{F}_{(3,2)}^{*}\right|$ with equality if and only if $\mathcal{F}$ is isomorphic to $\mathcal{F}_{(3,2)}^{*}$.

The main result in Chapter 4 is a characterization of intersecting families $\mathcal{F} \subseteq X^{(k)}$ where $c(\mathcal{F})=s \in\left(\left(\begin{array}{l}n-3 \\ k-2\end{array}\right), 4\left(\begin{array}{l}n-5 \\ k-3\end{array}\right)+\left(\begin{array}{l}n-5 \\ k-4\end{array}\right)\right]$. It is easy to show that $\left(\begin{array}{l}n-3 \\ k-2\end{array}\right) \leq 4\left(\begin{array}{l}n-5 \\ k-3\end{array}\right)+\left(\begin{array}{l}n-5 \\ k-4\end{array}\right)$ if and only if $n \leq 3 k-2$, so the theorem is vacuous for $n>3 k-2$. In Chapter 5 we show more. We show a $t$-intersecting generalization where the case $t=1$ says that for fixed $k$ and sufficiently large $n$, there does not exist an intersecting family $\mathcal{F} \subseteq X^{(k)}$ with $c(\mathcal{F})>\left(\begin{array}{l}n-3 \\ k-2\end{array}\right)$. 


\section{Chapter 2}

\section{Intersecting and $t$-cross-intersecting}

\section{Set Systems}

\subsection{Introduction}

Let $X=\{1,2, \cdots, n\}, \mathcal{P}(X)$ denote the set of all subsets of $X$ and $X^{(k)}$ denote the set of all subsets of $X$ of size $k$. For $1 \leq i \leq j \leq n$ we let $[i, j]=\{k \in X: i \leq k \leq j\}$. For $\mathcal{A} \subseteq \mathcal{P}(X)$ we let $\mathcal{A}_{i}=\{A \in \mathcal{A}:|A|=i\}$. For $A \subseteq X$ we let $A^{c}=X \backslash A$ and for $\mathcal{A} \subseteq \mathcal{P}(X)$ we let $\mathcal{A}^{c}=\left\{A^{c}: A \in \mathcal{A}\right\}$, the set of complements of sets in $\mathcal{A}$. If $r$ and $t$ are positive integers we say a family of sets $\mathcal{F} \subseteq \mathcal{P}(X)$ is $r$-intersecting if $|A \cap B| \geq r$ for each $A$ and $B \in \mathcal{F}$ and $\mathcal{F}_{1}, \mathcal{F}_{2} \subseteq \mathcal{P}(X)$ are $t$-cross-intersecting if $\left|A_{1} \cap A_{2}\right| \geq t$ for each $A_{1} \in \mathcal{F}_{1}$ and $A_{2} \in \mathcal{F}_{2}$. We usually say intersecting and cross-intersecting instead of 1-intersecting and 1-cross-intersecting. If $n+r$ is even we let

$$
\mathcal{F}(n, r)=\left\{F \subseteq X:|F| \geq \frac{n+r}{2}\right\}
$$

while if $n+r$ is odd we let

$$
\mathcal{F}(n, r)=\left\{F \subseteq X:|F| \geq \frac{n+r+1}{2} \text { or }|F|=\frac{n+r-1}{2} \text { and } 1 \notin F\right\} .
$$


The following theorem of Katona, with another proof by Kleitman [19], completely solves the problem of finding the largest $r$-intersecting subset of $\mathcal{P}(X)$.

Theorem 2.1.1 ([13]). If $\mathcal{A} \subseteq \mathcal{P}(X)$ is r-intersecting where $r \geq 2$ then $|\mathcal{A}| \leq|\mathcal{F}(n, r)|$ with equality holding if and only if $\mathcal{A}$ is isomorphic to $\mathcal{F}(n, r)$.

If $\mathcal{A}$ is 1-intersecting then obviously $|\mathcal{A}| \leq|\mathcal{F}(n, 1)|=2^{n-1}$, but there are many set systems for which equality holds.

A number of theorems about the maximum size of an intersecting family satisfying certain conditions have analogs or generalizations for cross-intersecting families. For example, the fundamental Erdős-Ko-Rado theorem has such a generalization due to Kleitman:

Theorem 2.1.2 (Erdös-Ko-Rado Theorem[3]). If $2 k \leq n$ and $\mathcal{F} \subseteq X^{(k)}$ is intersecting then $|\mathcal{F}| \leq\left(\begin{array}{l}n-1 \\ k-1\end{array}\right)$.

Theorem 2.1.3 ([20]). If $2 k \leq n$ and $\mathcal{F}_{1}, \mathcal{F}_{2} \subseteq X^{(k)}$ are cross-intersecting with $\left|\mathcal{F}_{1}\right| \leq\left|\mathcal{F}_{2}\right|$ then $\left|\mathcal{F}_{1}\right| \leq\left(\begin{array}{c}n-1 \\ k-1\end{array}\right)$.

For the proof of Theorem 2.1.6, one of our main results, we will need a characterization of when $\left|\mathcal{F}_{1}\right|=\left(\begin{array}{l}n-1 \\ k-1\end{array}\right)$ in Theorem 2.1.3. Though this is only a slight strengthening of Theorem 2.1.3, we state the results as Theorem 2.1.4 and Corollary 2.1.5 because they do not seem to have been stated before.

Theorem 2.1.4. Let $\mathcal{A} \subseteq X^{(a)}$ and $\mathcal{B} \subseteq X^{(b)}$ be cross-intersecting where $a+b<n$. If $|\mathcal{A}| \geq\left(\begin{array}{c}n-1 \\ a-1\end{array}\right)$ and $|\mathcal{B}| \geq\left(\begin{array}{c}n-1 \\ b-1\end{array}\right)$ then $\mathcal{A}=\left\{A \in X^{(a)}: j \in A\right\}$ and $\mathcal{B}=\left\{B \in X^{(b)}: j \in B\right\}$ for some $j \in X$.

A proof for Theorem 2.1.4 appears in Section 2.3. If we let $a=b$, we get the following characterization of equality in Theorem 2.1.3.

Corollary 2.1.5. If $2 k<n$ and $\mathcal{F}_{1}, \mathcal{F}_{2} \subseteq X^{(k)}$ are cross-intersecting with $\left(\begin{array}{c}n-1 \\ k-1\end{array}\right) \leq\left|\mathcal{F}_{1}\right| \leq\left|\mathcal{F}_{2}\right|$, then $\mathcal{F}_{1}=\mathcal{F}_{2}=\left\{F \in X^{(k)}: j \in F\right\}$ for some $j \in X$.

In this chapter we prove a cross-intersecting analog for Theorem 2.1.1: 
Theorem 2.1.6. Suppose $\mathcal{A}, \mathcal{B} \subseteq \mathcal{P}(X)$ are $t$-cross-intersecting where $t \geq 2$. If $|\mathcal{A}| \geq|\mathcal{B}| \geq$ $|\mathcal{F}(n, t)|$ then $\mathcal{A}=\mathcal{B}$ and both are isomorphic to $\mathcal{F}(n, t)$.

The following corollary of Theorem 2.1.6 was conjectured by Frankl [4] (a footnote was added in press in Frankl's paper saying the conjecture had been proved by Ahlswede and Katona, but Katona [17] says it was not published and perhaps was not ever done):

Corollary 2.1.7. If $\mathcal{A}, \mathcal{B} \subseteq \mathcal{P}(X)$ are t-cross-intersecting with $|\mathcal{A}| \geq|\mathcal{B}|$ then $|\mathcal{B}| \leq|\mathcal{F}(n, t)|$.

Clearly Corollary 2.1.7 follows immediately from Theorem 2.1.6 if $t \geq 2$. If $t=1$ and $A \in \mathcal{A}$ then $A^{c} \notin \mathcal{B}$, so clearly $|\mathcal{B}| \leq 2^{n-1}=|\mathcal{F}(n, 1)|$.

The other main result of this chapter is the following theorem which solves the problem of finding the maximum sum of the sizes of an $r_{1}$-intersecting and an $r_{2}$-intersecting family which are $t$-cross-intersecting:

Theorem 2.1.8. Suppose $\mathcal{A}, \mathcal{B} \subseteq \mathcal{P}(X)$ are t-cross-intersecting set systems such that $\mathcal{A}$ is $r_{1}$-intersecting and $\mathcal{B}$ is $r_{2}$-intersecting where $0<r_{1} \leq r_{2}$ and $t \geq 2$. Then

(1) If $t<\frac{r_{1}+r_{2}}{2}$ then $|\mathcal{A}|+|\mathcal{B}| \leq\left|\mathcal{F}\left(n, r_{1}\right)\right|+\left|\mathcal{F}\left(n, r_{2}\right)\right|$.

(2) If $t \geq \frac{r_{1}+r_{2}}{2}$ then $|\mathcal{A}|+|\mathcal{B}| \leq\left|\mathcal{F}\left(n, r_{1}\right)\right|+\left|\mathcal{F}\left(n, 2 t-r_{1}\right)\right|$.

Equality holds in (1) if and only if $\mathcal{A}$ is isomorphic to $\mathcal{F}\left(n, r_{1}\right)$ and $\mathcal{B}$ is isomorphic to $\mathcal{F}\left(n, r_{2}\right)$. Equality holds in (2) if and only if either

(a) $\mathcal{A}$ is isomorphic to $\mathcal{F}\left(n, r_{1}\right), \mathcal{B}$ is isomorphic to $\mathcal{F}\left(n, 2 t-r_{1}\right)$, and $\mathcal{A} \cup \mathcal{B}$ is isomorphic to $\mathcal{F}\left(n, r_{1}\right) \cup \mathcal{F}\left(n, 2 t-r_{1}\right)$ or

(b) $n+r_{1}$ is odd, $r_{1} \leq r_{2} \leq t=r_{1}+1$, and $\mathcal{A}=\mathcal{B}=\mathcal{F}(n, t)$.

The significance of the statement about $\mathcal{A} \cup \mathcal{B}$ in (a) is that if $n+r_{1}$ is odd then one symbol does not appear in any of the sets of size $\frac{n+r_{1}-1}{2}$ in $\mathcal{A}$ or in any of the sets of size $\frac{n+2 t-r_{1}-1}{2}$ in $\mathcal{B}$, and this must be the same symbol. The inequalities in the theorem hold for $t=1$ as well, but the statement characterizing equality in (2)(a) needs modification: if $r_{1}=r_{2}=t=1$ or if $r_{1}=1$ and $2 t-1>n$ (so that $\mathcal{B}=\emptyset$ ) then there are many set systems for which equality holds, because of the non-uniqueness of Theorem 2.1.1 for $r=1$. 
The proof we give for Theorem 2.1.8 uses theorems of Frankl, Hilton and Katona on intersecting and cross-intersecting families (all based on the Kruskal-Katona theorem), Harper's theorem on the Hamming distance between subsets of $\mathcal{P}(X)$, the Erdős-Ko-Rado Theorem, and a special case of Theorem 2.1.6 from this paper. The proof we give for Theorem 2.1.6 uses the shifting method Frankl and Füredi use to prove Harper's theorem in [7]. In Section 2.2 we define the orders LEX and COLEX, state the rest of the theorems we need, and discuss some of their relationships. The proofs of the two main results of this chapter - Theorem 2.1.6 and Theorem 2.1.8 - along with proofs of Theorem 2.1.4 and two lemmas, are in Section 2.3.

\subsection{Background}

If $\mathcal{A} \subseteq X^{(k)}$ we define the $g$-shadow $\partial^{(g)}(\mathcal{A})$ of $\mathcal{A}$ by $\partial^{(g)}(\mathcal{A})=\left\{G \in X^{(g)}: G \subseteq A\right.$ for some $A \in$ $\mathcal{A}\}$. We define the order COLEX on $X^{(k)}$ by $A<B$ if and only if $\max \{A \backslash B\}<\max \{B \backslash A\}$ and the order LEX on $X^{(k)}$ by $A<B$ if and only if $\min \{A \backslash B\}<\min \{B \backslash A\}$. So, for example, the order COLEX on $[1,5]^{(3)}$ is $123,124,134,234,125,135,235,145,245,345$, while the order LEX is $123,124,125,134,135,145,234,235,245,345$.

We denote the set of the first $m$ sets in LEX and COLEX in $X^{(k)}$ by $\mathcal{L}(k, m)$ and $\mathcal{C}(k, m)$ respectively. We refer to the first or last $m$ sets in LEX or COLEX on $X^{(k)}$ for any $m$ as an initial or terminal segment. If we take the complements of the sets in the order LEX on $[1,5]^{(3)}$ above we get $45,35,34,25,24,23,15,14,13,12$, suggesting the following lemma, which follows immediately from the definitions of LEX and COLEX.

Lemma 2.2.1. The complements of the sets in LEX order on $X^{(k)}$ are the sets in LEX in $X^{(n-k)}$ in reverse order, and also are the sets in $X^{(n-k)}$ in the COLEX order with the order $n<n-1<\cdots<2<1$ on $X$.

The order COLEX is particularly significant because of the following fundamental result.

Theorem 2.2.2 (Kruskal-Katona Theorem [21] [16]). If $\mathcal{A} \subseteq X^{(k)}$ then $\left|\partial^{(g)}(\mathcal{A})\right| \geq$ $\left|\partial^{(g)}(\mathcal{C}(k,|\mathcal{A}|))\right|$ for $g=1,2, \cdots, k-1$. If $|\mathcal{A}|=\left(\begin{array}{c}m \\ k\end{array}\right)$ for some integer $m \in[k, n]$ then equality holds if and only if $\mathcal{A}$ is isomorphic to $[1, m]^{(k)}$.

The following theorem of Hilton is equivalent to the inequality statement in the KruskalKatona theorem. 
Theorem 2.2.3 ([12]). If $\mathcal{A} \subseteq X^{(a)}$ and $\mathcal{B} \subseteq X^{(b)}$ are cross-intersecting then so are $\mathcal{L}(a,|\mathcal{A}|)$ and $\mathcal{L}(b,|\mathcal{B}|)$.

We sketch the argument that Theorem 2.2.3 follows from the Kruskal-Katona theorem. Note that $\mathcal{A}$ and $\mathcal{B}$ are cross-intersecting if and only if $\partial^{(b)}\left(\mathcal{A}^{c}\right) \cap \mathcal{B}=\emptyset$. So if $\mathcal{A}$ and $\mathcal{B}$ are cross-intersecting then $\left|\partial^{(b)}\left(\mathcal{A}^{c}\right)\right|+|\mathcal{B}| \leq\left(\begin{array}{l}n \\ b\end{array}\right)$. By Lemma 2.2.1, $\mathcal{L}(a,|\mathcal{A}|)^{c}$ is an initial segment

in COLEX on $X^{(n-a)}$ with the reverse order on $X$. So $\partial^{(b)}\left(\mathcal{L}(a,|\mathcal{A}|)^{c}\right)$ is an initial segment in COLEX on $X^{(b)}$ with the reverse order on $X$, but is also a terminal segment in LEX on $X^{(b)}$ (with the usual order on $X$ ). Obviously $\mathcal{L}(b,|\mathcal{B}|)$ is an initial segment in LEX on $X^{(b)}$. By the Kruskal-Katona theorem

$$
\left|\partial^{(b)}\left(\mathcal{L}(a,|\mathcal{A}|)^{c}\right)\right|+|\mathcal{L}(b,|\mathcal{B}|)| \leq\left|\partial^{(b)}\left(\mathcal{A}^{c}\right)\right|+|\mathcal{B}| \leq\left(\begin{array}{c}
n \\
b
\end{array}\right)
$$

so $\partial^{(b)}\left(\mathcal{L}(a,|\mathcal{A}|)^{c}\right) \cap \mathcal{L}(b,|\mathcal{B}|)=\emptyset$ and $\mathcal{L}(a,|\mathcal{A}|)$ and $\mathcal{L}(b,|\mathcal{B}|)$ are cross-intersecting.

The following lemma has appeared in various forms in many papers:

Lemma 2.2.4. Let $\emptyset \neq \mathcal{A} \subseteq X^{(k)}$ and $g<k$ be a positive integer. Then $\left|\partial^{(g)}(\mathcal{A})\right| \geq \frac{\left(\begin{array}{l}n \\ g\end{array}\right)}{\left(\begin{array}{c}n \\ k\end{array}\right)}|\mathcal{A}|$ with equality holding if and only if $\mathcal{A}=X^{(k)}$.

Proof Consider the bipartite graph $G$ with vertex bipartition $\mathcal{A} \cup \partial^{(g)}(\mathcal{A})$, with $A \in \mathcal{A}$ and $B \in \partial^{(g)}(\mathcal{A})$ adjacent if and only if $B \subseteq A$. The degree in $G$ of each set $A$ in $\mathcal{A}$ is $\left(\begin{array}{l}k \\ g\end{array}\right)$, and is at most $\left(\begin{array}{c}n-g \\ k-g\end{array}\right)$ for each set $B$ in $\partial^{(g)}(\mathcal{A})$. Hence

$$
\left|\partial^{(g)}(\mathcal{A})\right| \geq \frac{\left(\begin{array}{l}
k \\
g
\end{array}\right)}{\left(\begin{array}{l}
n-g \\
k-g
\end{array}\right)}|\mathcal{A}|=\frac{\left(\begin{array}{l}
n \\
g
\end{array}\right)}{\left(\begin{array}{l}
n \\
k
\end{array}\right)}|\mathcal{A}|
$$

with equality holding if and only if each set $A$ in $X^{(k)}$ which contains a set $B$ in $\partial^{(g)}(\mathcal{A})$ is in $\mathcal{A}$. Since the similarly defined bipartite graph with vertex bipartition $X^{(k)} \cup X^{(g)}$ is connected, this happens if and only if $\mathcal{A}=X^{(k)}$ (and $\left.\partial^{(g)}(\mathcal{A})=X^{(g)}\right)$.

We will need two lemmas of Frankl about cross-intersecting families. One (Lemma 2.2.5) follows easily from Lemma 2.2.4, while the other (Lemma 2.2.7) follows from a theorem of Katona (Theorem 2.2.6). 
Lemma 2.2.5 ([6], Proposition 1.3). Let $\mathcal{A} \subseteq X^{(a)}$ and $\mathcal{B} \subseteq X^{(b)}$ be cross-intersecting, where $a \geq b$ and $a+b<n$. Then $|\mathcal{A}|+|\mathcal{B}| \leq\left(\begin{array}{l}n \\ a\end{array}\right)$ with equality holding if and only if either $\mathcal{A}=X^{(a)}$ and $\mathcal{B}=\emptyset$ or $a=b, \mathcal{A}=\emptyset$, and $\mathcal{B}=X^{(a)}$.

Proof If $\mathcal{A}$ and $\mathcal{B}$ are an optimal pair of such families then

$$
|\mathcal{A}|+|\mathcal{B}|=\left(\begin{array}{l}
n \\
a
\end{array}\right)+|\mathcal{B}|-\left|\partial^{(a)}\left(\mathcal{B}^{c}\right)\right|
$$

because $\mathcal{A} \cap \partial^{(a)}\left(\mathcal{B}^{c}\right)=\emptyset$. Since $a<n-b$, by Lemma 2.2.4,

$$
\left|\partial^{(a)}\left(\mathcal{B}^{c}\right)\right| \geq \frac{\left(\begin{array}{c}
n \\
a
\end{array}\right)}{\left(\begin{array}{c}
n \\
n-b
\end{array}\right)}\left|\mathcal{B}^{c}\right|
$$

with equality holding if and only if $\mathcal{B}^{c}=\emptyset$ or $\mathcal{B}^{c}=X^{(n-b)}$. Since $a<n-b$ and $a+(n-b) \geq n$, $\frac{\left(\begin{array}{c}n \\ a\end{array}\right)}{\left(\begin{array}{c}n \\ n-b\end{array}\right)} \geq 1$ with equality if and only if $a=b$. Hence $\left|\partial^{(a)}\left(\mathcal{B}^{c}\right)\right| \geq\left|\mathcal{B}^{c}\right|$ with equality if and only if $\mathcal{B}=\emptyset\left(\right.$ and $\left.\mathcal{A}=X^{(a)}\right)$ or $a=b, \mathcal{B}=X^{(b)}$, and $\mathcal{A}=\emptyset$.

The following theorem is a special case of a theorem of Katona which has been an essential ingredient in the proofs of several results about intersecting families.

Theorem 2.2.6 ([13]). Suppose $\emptyset \neq \mathcal{A} \subseteq X^{(k)}$ is t-intersecting. Then $\left|\partial^{(k-t)}(\mathcal{A})\right| \geq|\mathcal{A}|$ with equality holding if and only if $\mathcal{A}=Y^{(k)}$ for some $Y \in X^{(2 k-t)}$.

Frankl used this theorem and techniques similar to those used to prove Theorem 2.2.3 from the Kruskal-Katona theorem to prove the following lemma:

Lemma 2.2.7 ([6], Proposition 1.4). Let $k$ and $t$ be positive integers with $2 k+t<n$. If $\mathcal{A} \subseteq X^{(k+t)}$ and $\mathcal{B} \subseteq X^{(k)}$ are cross-intersecting and $\mathcal{A}$ is t-intersecting then $|\mathcal{A}|+|\mathcal{B}| \leq\left(\begin{array}{l}n \\ k\end{array}\right)$ with equality holding if and only if either

(1) $\mathcal{A}=\emptyset$ and $\mathcal{B}=X^{(k)}$ or

(2) $\mathcal{A}=\left\{A \in X^{(k+t)}: T \subseteq A\right\}$ and $\mathcal{B}=\left\{B \in X^{(k)}: B \cap T \neq \emptyset\right\}$ for some $T \in X^{(t)}$. 
We will also need the following lemma, in the same spirit as Lemma 2.2.7:

Lemma 2.2.8. Let $\mathcal{A} \subseteq X^{(a)}$ and $\mathcal{B} \subseteq X^{(b)}$ be cross-intersecting where $\mathcal{A}$ is intersecting and $b<a \leq \frac{n}{2}$. Then $|\mathcal{A}|+|\mathcal{B}| \leq\left(\begin{array}{c}n-1 \\ a-1\end{array}\right)+\left(\begin{array}{c}n-1 \\ b-1\end{array}\right)$ with equality holding if and only if either

(1) $\mathcal{A}=\left\{A \in X^{(a)}: k \in A\right\}$ and $\mathcal{B}=\left\{B \in X^{(b)}: k \in B\right\}$ for some $k \in X$ or

(2) $a=b+1, A=\emptyset$, and $\mathcal{B}=X^{(b)}$.

A proof for Lemma 2.2.8 is in Section 2.3.

To characterize equality in Theorem 2.1.8 we will use the following lemma, which is a special case of Theorem 2.1.6. We include it because it has an interesting proof given in Section 2.3, using different ideas than our proof of Theorem 2.1.6.

Lemma 2.2.9. Suppose $\mathcal{A}, \mathcal{B} \subseteq \mathcal{P}(X)$ are both $t-1$ intersecting and $\mathcal{A}$ and $\mathcal{B}$ are $t$-crossintersecting where $t \geq 2$ and $n+t$ is even. If $|\mathcal{A}|=|\mathcal{B}|=|\mathcal{F}(n, t)|$ then $\mathcal{A}=\mathcal{B}=\mathcal{F}(n, t)$.

Finally, we will need a result of Harper about Hamming distance between sets. If $A$ and $C$ are subsets of $X$ we define the distance $d(A, C)$ to be $|A \triangle C|$ where $A \triangle C$ is the symmetric difference of $A$ and $C$, i.e., the set of elements appearing in precisely one of $A$ and $C$. We define the distance $d(\mathcal{A}, \mathcal{C})$ between families $\mathcal{A}, \mathcal{C} \subseteq \mathcal{P}(X)$ to be the minimum of $d(A, C)$ such that $A \in \mathcal{A}$ and $C \in \mathcal{C}$. If $G \subseteq X$ we define the sphere $\mathcal{S}(G, m)$ with center $G$ and radius $m$ by $\mathcal{S}(G, m)=\{A \subseteq X: d(A, G) \leq m\}$. If $\mathcal{H} \subseteq \mathcal{P}(X)$, we say $\mathcal{H}$ is a Hamming ball with center $G$ if $\mathcal{S}(G, m) \subseteq \mathcal{H} \subseteq \mathcal{S}(G, m+1)$ for some nonnegative integer $m$.

Theorem 2.2.10 ([11]). If $\mathcal{A}, \mathcal{B} \subseteq \mathcal{P}(X)$ are non-empty families then there exists a Hamming ball $\mathcal{A}_{0}$ with center $X$ and a Hamming ball $\mathcal{B}_{0}$ with center $\emptyset$ such that $|\mathcal{A}|=\left|\mathcal{A}_{0}\right|,|\mathcal{B}|=\left|\mathcal{B}_{0}\right|$ and $d\left(\mathcal{A}_{0}, \mathcal{B}_{0}\right) \geq d(\mathcal{A}, \mathcal{B})$.

For a proof of Theorem 2.2.10 see [11], [7] or [2].

Corollary 2.2.11. If $\mathcal{A}, \mathcal{B} \subseteq \mathcal{P}(X)$ are t-cross-intersecting families then there exist Hamming balls $\mathcal{A}_{0}$ and $\mathcal{B}_{0}$ with center $X$ such that $\left|\mathcal{A}_{0}\right|=|\mathcal{A}|,\left|\mathcal{B}_{0}\right|=|\mathcal{B}|$, and $\mathcal{A}_{0}, \mathcal{B}_{0}$ are $t$-crossintersecting. 
Proof Since $d\left(\mathcal{A}^{c}, \mathcal{B}\right) \geq t$, by Theorem 2.2.10 there exist Hamming balls $\mathcal{A}_{1}$ centered at $\emptyset$ and $\mathcal{B}_{0}$ centered at $X$ such that $\left|\mathcal{A}_{1}\right|=|\mathcal{A}|,\left|\mathcal{B}_{0}\right|=|\mathcal{B}|$ and $d\left(\mathcal{A}_{1}, \mathcal{B}_{0}\right) \geq t$. Let $\mathcal{A}_{0}=\mathcal{A}_{1}^{c}$. We claim $\mathcal{A}_{0}, \mathcal{B}_{0}$ are $t$-cross-intersecting. Let $i$ be the smallest size of a set in $\mathcal{A}_{0}$ and $j$ be the smallest size of a set in $\mathcal{B}_{0}$. If $i+j \geq n+t$ then $\mathcal{A}_{0}$ and $\mathcal{B}_{0}$ are obviously $t$-cross-intersecting. Since $\mathcal{A}_{1}$ and $\mathcal{B}_{0}$ are Hamming balls with $d\left(\mathcal{A}_{1}, \mathcal{B}_{0}\right) \geq t$, we must have $j-(n-i) \geq t-1$, so we need only consider the possibility $j-(n-i)=t-1$. In this case, if $E \in\left(\mathcal{A}_{1}\right)_{n-i}$ and $D \in\left(\mathcal{B}_{0}\right)_{j}$ then $D$ has at least $t$ elements not in $E$ (and $E$ has at least one element not in $D$, so in fact $d(E, D) \geq t+1)$, so $\left|E^{c} \cap D\right| \geq t$ as required.

\subsection{Proofs}

Proof of Theorem 2.1.4 By Theorem 2.2.3 $\mathcal{L}(a,|\mathcal{A}|)$ and $\mathcal{L}(b,|\mathcal{B}|)$ are cross-intersecting. Since $|\mathcal{A}| \geq\left(\begin{array}{c}n-1 \\ a-1\end{array}\right)$ and $|\mathcal{B}| \geq\left(\begin{array}{c}n-1 \\ b-1\end{array}\right), \mathcal{L}(a,|\mathcal{A}|)$ and $\mathcal{L}(b,|\mathcal{B}|)$ contain all subsets of $X^{(a)}$ and $X^{(b)}$ respectively containing 1 , so neither contains any sets not containing 1 . Hence $|\mathcal{A}|=\left(\begin{array}{c}n-1 \\ a-1\end{array}\right)$ and $|\mathcal{B}|=\left(\begin{array}{c}n-1 \\ b-1\end{array}\right)$. As in the proof of Theorem 2.2.3, $\mathcal{B} \subseteq X^{(b)} \backslash \partial^{(b)}\left(\mathcal{A}^{c}\right)$, so $\left|\partial^{(b)}\left(\mathcal{C}\left(n-a,\left|\mathcal{A}^{c}\right|\right)\right)\right| \leq$ $\left|\partial^{(b)}\left(\mathcal{A}^{c}\right)\right| \leq\left(\begin{array}{c}n \\ b\end{array}\right)-\left(\begin{array}{c}n-1 \\ b-1\end{array}\right)=\left(\begin{array}{c}n-1 \\ b\end{array}\right)=\left|\partial^{(b)}\left(\mathcal{C}\left(n-a,\left|\mathcal{A}^{c}\right|\right)\right)\right|$. Since $\mathcal{A}^{c} \subseteq X^{(n-a)}$ and $\left|\mathcal{A}^{c}\right|=\left(\begin{array}{c}n-1 \\ a-1\end{array}\right)=$ $\left(\begin{array}{l}n-1 \\ n-a\end{array}\right)$, by the equality part of the Kruskal-Katona theorem, $\mathcal{A}^{c}$ is isomorphic to $\mathcal{C}(n-a,|\mathcal{A}|)$.

Hence $\mathcal{A}$ is isomorphic to $\mathcal{L}(a,|\mathcal{A}|)=\left\{A \in X^{(a)}: 1 \in A\right\}$, and clearly $\mathcal{B}$ has the required form as well.

Proof of Lemma 2.2.8 Let $\mathcal{A} \subseteq X^{(a)}$ and $\mathcal{B} \subseteq X^{(b)}$ be cross-intersecting where $\mathcal{A}$ is intersecting, $b<a \leq \frac{n}{2}$, and $|\mathcal{A}|+|\mathcal{B}|$ has maximum possible value. By Theorem 2.2.3, $\mathcal{L}(a,|\mathcal{A}|)$ and $\mathcal{L}(b,|\mathcal{B}|)$ are cross-intersecting. Since $\mathcal{A}$ is intersecting, by the Erdős-Ko-Rado theorem, $|\mathcal{A}| \leq\left(\begin{array}{c}n-1 \\ a-1\end{array}\right)$. Hence every set in $\mathcal{L}(a,|\mathcal{A}|)$ contains 1 . By the maximality of $|\mathcal{A}|+|\mathcal{B}|$, every set

in $X^{(b)}$ containing 1 must be in $\mathcal{L}(b,|\mathcal{B}|)$. Let $\mathcal{G}=\{G \in \mathcal{L}(b,|\mathcal{B}|): 1 \notin G\}$. We think of $\mathcal{G}$ as a subset of $[2, n]$ and consider $\mathcal{G}^{c} \subseteq[2, n]^{(n-1-b)}$ and $\partial^{(a-1)}\left(\mathcal{G}^{c}\right) \subseteq[2, n]^{(a-1)}$. We have

$$
|\mathcal{A}|+|\mathcal{B}|=|\mathcal{L}(b,|\mathcal{B}|)|+|\mathcal{L}(a,|\mathcal{A}|)|=\left(\begin{array}{c}
n-1 \\
b-1
\end{array}\right)+\left|\mathcal{G}^{c}\right|+\left(\begin{array}{c}
n-1 \\
a-1
\end{array}\right)-\left|\partial^{(a-1)}\left(\mathcal{G}^{c}\right)\right|
$$

since if $F \in \partial^{(a-1)}\left(\mathcal{G}^{c}\right)$ then $(\{1\} \cup F) \notin \mathcal{L}(a,|\mathcal{A}|)$. 
By Lemma 2.2.4,

$$
\left|\partial^{(a-1)}\left(\mathcal{G}^{c}\right)\right| \geq \frac{\left(\begin{array}{c}
n-1 \\
a-1
\end{array}\right)}{\left(\begin{array}{c}
n-1 \\
n-b-1
\end{array}\right)}\left|\mathcal{G}^{c}\right|
$$

Since $(a-1)+(n-b-1)=(n-1)+[a-(b+1)] \geq n-1$ and $(a-1) \leq \frac{n}{2}-1=n-1-\frac{n}{2} \leq$ $(n-1)-(b+1)<n-b-1$, it follows that $\left|\partial^{(a-1)}\left(\mathcal{G}^{c}\right)\right| \geq\left|\mathcal{G}^{c}\right|$ with equality holding if and only if either $\mathcal{G}^{c}=\emptyset$ or $(a-1)+(n-b-1)=n-1$ and $\mathcal{G}^{c}=[2, n]^{(n-1-b)}$ (again using the fact that the auxiliary bipartite graph is connected). The first of these gives us case (1) and the second gives us case (2).

Proof of Lemma 2.2.9 For each $i \in\left[t-1, \frac{n+t-2}{2}\right]$ we consider the set systems $\left(\mathcal{A}^{c}\right)_{n-i}$ and $\left(\mathcal{B}^{c}\right)_{i-t+1}$. Since $\mathcal{A}_{i}$ and $\mathcal{B}_{n-i+t-1}$ are $t$-cross-intersecting and $(n-i)+(i-t+1)-n+t=$ 1 , $\left(\mathcal{A}^{c}\right)_{n-i}$ and $\left(\mathcal{B}^{c}\right)_{i-t+1}$ are cross-intersecting. Furthermore, since $\mathcal{A}_{i}$ is $(t-1)$-intersecting and $2(n-i)-n+(t-1)=n-2 i+t-1,\left(\mathcal{A}^{c}\right)_{n-i}$ is $(n-2 i+t-1)$-intersecting. Since $(n-2 i+t-1)+(i-t+1)=n-i$ and $2(i-t+1)+(n-2 i+t-1)=n-t+1<n$, the set systems $\left(\mathcal{A}^{c}\right)_{n-i}$ and $\left(\mathcal{B}^{c}\right)_{i-t+1}$ satisfy the hypotheses for Lemma 2.2 .7 with $i-t+1$ in place of $k$ and $n-2 i+t-1$ in place of $t$. Hence

$$
\left|\mathcal{A}_{i}\right|+\left|\mathcal{B}_{n-i+t-1}\right| \leq\left(\begin{array}{c}
n \\
i-t+1
\end{array}\right)
$$

with equality if and only if either

(1) $\mathcal{A}_{i}=\emptyset$ and $\mathcal{B}_{n-i+t-1}=X^{(n-i+t-1)}$ or

(2) $\mathcal{A}_{i}=\left\{A \in X^{(i)}: T \cap A=\emptyset\right\}$ and $\mathcal{B}_{n-i+t-1}=\left\{B \in X^{(n-i+t-1)}: T \nsubseteq B\right\}$ for some $T \in X^{(n-2 i+t-1)}$.

Thus $\sum_{i=t-1}^{\frac{n+t-2}{2}}\left|\mathcal{A}_{i}\right|+\sum_{j=\frac{n+t}{2}}^{n}\left|\mathcal{B}_{j}\right| \leq \sum_{i=t-1}^{\frac{n+t-2}{2}}\left(\begin{array}{c}n \\ i-t+1\end{array}\right)=|\mathcal{F}(n, t)|$ with the exact same inequality also holding with $\mathcal{B}_{i}$ in place of $\mathcal{A}_{i}$ and $\mathcal{A}_{j}$ in place of $\mathcal{B}_{j}$.

Adding these two inequalities then gives

$$
2|\mathcal{F}(n, t)|=\sum_{i=t-1}^{n}\left(\left|\mathcal{A}_{i}\right|+\left|\mathcal{B}_{i}\right|\right) \leq 2|\mathcal{F}(n, t)|
$$


So equality must hold in $(*)$ for each $i$. By way of contradiction, suppose condition (2) holds for some $i \in\left[t-1, \frac{n+t-2}{2}\right]$. Since $n-i+t-1 \geq \frac{n+t}{2}$, there is some set of size at least $\frac{n+t}{2}$ which is not in $\mathcal{B}$. Since $|\mathcal{B}|=|\mathcal{F}(n, t)|$, for some $j \in\left[t-1, \frac{n+t-2}{2}\right]$ there is a set of size $j$ which

is in $\mathcal{B}$. So by equality condition (2) (with $\mathcal{A}$ and $\mathcal{B}$ reversed), $\mathcal{B}_{j}=\left\{B \in X^{(j)}: R \cap B=\emptyset\right\}$ for some $R \in X^{(n-2 j+t-1)}$, while $\mathcal{A}_{i}=\left\{A \in X^{(i)}: T \cap A=\emptyset\right\}$ for some $T \in X^{(n-2 i+t-1)}$. Without loss of generality suppose $i \leq j$. Since $\left|R^{c}\right|=2 j-t+1>i+j-t$, for each $A \in \mathcal{A}_{i}$ there exists $B \in \mathcal{B}_{j}$ such that $|A \cap B|<t$, a contradiction. Since condition (2) cannot hold for any $i$, we must have $\mathcal{A}_{i}=\emptyset$ for all $i \in\left[t-1, \frac{n+t-2}{2}\right]$ and $\mathcal{A}=\mathcal{F}(n, t)$. So $\mathcal{B}=\mathcal{F}(n, t)$ as well.

Proof of Theorem 2.1.8 Suppose $t<\frac{r_{1}+r_{2}}{2}$. By Theorem 2.1.1, $|\mathcal{A}| \leq\left|\mathcal{F}\left(n, r_{1}\right)\right|$ and $|\mathcal{B}| \leq$ $\left|\mathcal{F}\left(n, r_{2}\right)\right|$ with equality holding if and only if $\mathcal{A}$ is isomorphic to $\mathcal{F}\left(n, r_{1}\right)$ and $\mathcal{B}$ is isomorphic to $\mathcal{F}\left(n, r_{2}\right)$. If $\mathcal{A}$ is isomorphic to $\mathcal{F}\left(n, r_{1}\right)$ and $\mathcal{B}$ is isomorphic to $\mathcal{F}\left(n, r_{2}\right)$ then the greatest integer $t^{\prime}$ for which $\mathcal{A}$ and $\mathcal{B}$ are $t^{\prime}$-cross-intersecting is $\frac{r_{1}+r_{2}-1}{2}$ if $r_{1}+r_{2}$ is odd and $\frac{r_{1}+r_{2}}{2}$ if $r_{1}$ and $r_{2}$ are even, so $\mathcal{A}$ and $\mathcal{B}$ are $t$-cross-intersecting. If $r_{1}$ and $r_{2}$ are odd and $i$ is the element of $X$ missing from all $\frac{n+r_{1}-1}{2}$-sets in $\mathcal{A}$ and $j$ is the element missing from all $\frac{n+r_{2}-1}{2}$-sets in $\mathcal{B}$, then $\mathcal{A}$ and $\mathcal{B}$ are $\frac{r_{1}+r_{2}}{2}$-cross-intersecting if $i=j$ and $\frac{r_{1}+r_{2}-2}{2}$-cross-intersecting if $i \neq j$, verifying the equality statement in the theorem if $t<\frac{r_{1}+r_{2}}{2}$.

If $t=\frac{r_{1}+r_{2}}{2}$ the argument is exactly as above except that if $n+r_{1}$ is odd $\left(n+r_{2}\right.$ will also be odd) then we need the extra condition about $\mathcal{A} \cup \mathcal{B}$ in the statement about equality.

Now suppose that $t>\frac{r_{1}+r_{2}}{2}$ and that $\left|\mathcal{A}^{\prime}\right|+\left|\mathcal{B}^{\prime}\right|$ is maximal such that $\mathcal{A}^{\prime}$ is $r_{1}$-intersecting, $\mathcal{B}^{\prime}$ is $r_{2}$-intersecting, and $\mathcal{A}^{\prime}$ and $\mathcal{B}^{\prime}$ are $t$-cross-intersecting. By Corollary 2.2.11 there exist Hamming balls $\mathcal{A}$ and $\mathcal{B}$ with center $X$ such that $|\mathcal{A}|=\left|\mathcal{A}^{\prime}\right|,|\mathcal{B}|=\left|\mathcal{B}^{\prime}\right|$, and $\mathcal{A}, \mathcal{B}$ are $t$-crossintersecting. Let $i$ be the smallest size of a set in $\mathcal{A}$ and $j$ be smallest size of a set in $\mathcal{B}$.

If $i+j>n+t$ then, since $t>\frac{r_{1}+r_{2}}{2}, i+j \geq n+t+1>n+\frac{r_{1}+r_{2}}{2}+1=\frac{n+r_{1}+1}{2}+\frac{n+r_{2}+1}{2}$, so either $\mathcal{A}$ is $\left(r_{1}+1\right)$-intersecting or $\mathcal{B}$ is $\left(r_{2}+1\right)$-intersecting, which means we can enlarge either $\mathcal{A}$ or $\mathcal{B}$ and still satisfy all hypotheses, contradicting the maximality assumption on $|\mathcal{A}|+|\mathcal{B}|$.

If $i+j \leq n+t-2$ then $\mathcal{A}$ and $\mathcal{B}$ are not $t$-cross-intersecting. So $i+j=n+t$ or $i+j=n+t-1$. We can assume $i \leq j$ (since otherwise we could just switch $\mathcal{A}$ and $\mathcal{B}$ ). If $i \leq \frac{n+r_{1}-2}{2}$ then, since $X^{(i+1)} \subseteq \mathcal{A}, \mathcal{A}$ is not $r_{1}$-intersecting. So we can assume $\frac{n+r_{1}-1}{2} \leq i \leq j$.

Case 1. $n+r_{1}$ is even

Suppose $i+j=n+t$. Since $|\mathcal{A}|+|\mathcal{B}|=\sum_{m=i+1}^{n}\left(\begin{array}{c}n \\ m\end{array}\right)+\sum_{m=j+1}^{n}\left(\begin{array}{c}n \\ m\end{array}\right)+\left|\mathcal{A}_{i}\right|+\left|\mathcal{B}_{j}\right|$ and 
$\frac{n+r_{1}}{2} \leq i \leq j$, to maximize $|\mathcal{A}|+|\mathcal{B}|$ we must have $\mathcal{A}_{i}=X^{(i)}, \mathcal{B}_{j}=X^{(j)}, i=\frac{n+r_{1}}{2}$, and $j=\frac{n+2 t-r_{1}}{2}$, so $\mathcal{A}=\mathcal{F}\left(n, r_{1}\right)$ and $\mathcal{B}=\mathcal{F}\left(n, 2 t-r_{1}\right)$. Now suppose $i+j=n+t-1$. Since $\mathcal{A}_{i}$ and $\mathcal{B}_{n+t-i-1}$ are $t$-cross-intersecting, and $(n-i)+(i-t+1)-n+t=1,\left(\mathcal{A}^{c}\right)_{n-i}$ and $\left(\mathcal{B}^{c}\right)_{i-t+1}$ are non-empty and cross-intersecting. Since $(n-i)+(i-t+1)=n-t+1<n$ and $n-i \geq i-t+1$, by Lemma 2.2.5 $\left|\left(\mathcal{A}^{c}\right)_{n-i}\right|+\left|\left(\mathcal{B}^{c}\right)_{i-t+1}\right|<\left(\begin{array}{c}n \\ n-i\end{array}\right)$ (since the conditions for equality in Lemma 2.2.5 do not hold), and $|\mathcal{A}|+|\mathcal{B}|$ is smaller than if $i+j=n+t$.

Case 2. $n+r_{1}$ is odd

Precisely the same argument used in Case 1 shows that if $i$ is the smallest size of a set in $\mathcal{A}$ and if $i \geq \frac{n+r_{1}+1}{2}$ then $|\mathcal{A}|+|\mathcal{B}|$ is maximized only when $i=\frac{n+r_{1}+1}{2}, \mathcal{A}=\mathcal{S}\left(X, \frac{n-r_{1}-1}{2}\right)=$ $\mathcal{F}\left(n, r_{1}+1\right), \mathcal{B}=\mathcal{S}\left(X, \frac{n-2 t+r_{1}+1}{2}\right)=\mathcal{F}\left(n, 2 t-r_{1}-1\right)$. But we still have the possibility $i=$ $\frac{n+r_{1}-1}{2}$. For this value of $i$ we again consider the cross-intersecting families $\left(\mathcal{A}^{c}\right)_{n-i}$ and $\left(\mathcal{B}^{c}\right)_{i-t+1}$. We now allow the possibility $\left(\mathcal{A}^{c}\right)_{n-i}=\emptyset$, because that includes the optimal configuration of Case 2 given above (when $i \geq \frac{n+r_{1}+1}{2}$ ). Since $\mathcal{A}_{i}$ is $r_{1}$-intersecting and $2(n-i)-n+r_{1}=1$, $\left(\mathcal{A}^{c}\right)_{n-i}$ is intersecting, so now we can use Lemma 2.2.8 (instead of Lemma 2.2.5 as before) once we verify that the other hypotheses are satisfied. Since $t>\frac{r_{1}+r_{2}}{2} \geq r_{1}$ we have $(i-t+1)+1 \leq$ $\left(i-r_{1}\right)+1=n-i=\frac{n-r_{1}+1}{2} \leq \frac{n}{2}$. So by Lemma 2.2.8, $|\mathcal{A}|+|\mathcal{B}| \leq\left(\begin{array}{c}n-1 \\ n-i-1\end{array}\right)+\left(\begin{array}{c}n-1 \\ i-t\end{array}\right)$ with equality holding if and only if either

(i) $\mathcal{A}_{i}=\left\{A \in X^{(i)}: k \notin A\right\}$ and $\mathcal{B}_{n-i+t-1}=\left\{B \in X^{(n-i+t-1)}: k \notin B\right\}$ for some $k \in X$ or

(ii) $n-i=(i-t+1)+1, \mathcal{A}_{i}=\emptyset$ and $\mathcal{B}_{n-i+t-1}=X^{(n-i+t-1)}$

The first of these gives the optimal configuration of (2)(a), and the second of these gives $t=2 i-n+2=r_{1}+1$. Since $t>\frac{r_{1}+r_{2}}{2}$ we must have $r_{1} \leq r_{2} \leq t=r_{1}+1$, so $\mathcal{A}=\mathcal{B}=\mathcal{F}(n, t)$, the optimal configuration of (2)(b).

As in Case 1, by the uniqueness of the optimal configuration in Theorem 2.1.1, if $\mathcal{A}$ is isomorphic to $\mathcal{F}\left(n, r_{1}\right)$ then so is $\mathcal{A}^{\prime}$ (and hence $\mathcal{B}$ is isomorphic to $\mathcal{B}^{\prime}$ as well). But in the case $r_{1} \leq r_{2} \leq t=r_{1}+1$ and $\mathcal{A}=\mathcal{B}=\mathcal{F}(n, t)$, since $\mathcal{A}^{\prime}$ is only $(t-1)$-intersecting, we need Lemma 2.2.9 to conclude that $\mathcal{A}^{\prime}=\mathcal{B}^{\prime}=\mathcal{F}(n, t)$.

Proof of Theorem 2.1.6 If $\mathcal{A}, \mathcal{B} \subseteq \mathcal{P}(X)$ are $t$-cross-intersecting with $|\mathcal{A}| \geq|\mathcal{B}| \geq|\mathcal{F}(n, t)|$, by Corollary 2.2.11 there exist $t$-cross-intersecting Hamming balls $\mathcal{A}_{0}$ and $\mathcal{B}_{0}$ with center $X$ with $\left|\mathcal{A}_{0}\right| \geq\left|\mathcal{B}_{0}\right| \geq|\mathcal{F}(n, t)|$. If $n+t$ is even then $\mathcal{A}_{0}$ and $\mathcal{B}_{0}$ both contain $\mathcal{F}(n, t)$, and since they are $t$-cross-intersecting, they clearly must both be equal to $\mathcal{F}(n, t)$. If $n+t$ is odd, then $\left|\left(\mathcal{A}_{0}\right)_{\frac{n+t-1}{2}}\right|$ and $\left|\left(\mathcal{B}_{0}\right)_{\frac{n+t-1}{2}}\right|$ are both greater than or equal to $\left|\mathcal{F}(n, t)_{\frac{n+t-1}{2}}\right|=\left(\begin{array}{c}n-1 \\ \frac{n+t-1}{2}\end{array}\right)=\left(\begin{array}{c}n-1 \\ \frac{n-t-1}{2}\end{array}\right)$. But 
$\left[\left(\mathcal{A}_{0}\right)_{\frac{n+t-1}{2}}\right]^{c}$ and $\left[\left(\mathcal{B}_{0}\right)_{\frac{n+t-1}{2}}\right]^{c}$ are cross-intersecting families of subsets of $X^{\left(\frac{n-t+1}{2}\right)}$. So by Corollary 2.1.5 (with $\left.k=\frac{n-t+1}{2}\right),\left|\left(\mathcal{A}_{0}\right)_{\frac{n+t-1}{2}}\right|=\left|\left(\mathcal{B}_{0}\right)_{\frac{n+t-1}{2}}\right|=\left(\begin{array}{c}n-1 \\ \frac{n+t-1}{2}\end{array}\right)$, and in fact $\mathcal{A}_{0}=\mathcal{B}_{0}$ and both are isomorphic to $\mathcal{F}(n, t)$.

(1) $n+t$ is even.

Suppose $\mathcal{A}$ is not $\mathcal{F}(n, t)$. Then we can find $A_{0} \in \mathcal{A}$ with $\left|\mathcal{A}_{0}\right|<\frac{n+t}{2}$ and $A_{0}^{\prime} \notin \mathcal{A}$ such that $\left|A_{0}\right|<\left|A_{0}^{\prime}\right|$, and we choose $A_{0}$ and $A_{0}^{\prime}$ such that $d\left(A_{0}, A_{0}^{\prime}\right)$ is minimum. Set $U=A_{0} \backslash A_{0}^{\prime}, V=$ $A_{0}^{\prime} \backslash A_{0}$, so $0 \leq|U|<|V|$.

For each $A \in \mathcal{A}$, we define $T_{\mathcal{A}}(U, V ; A)$ by

$$
T_{\mathcal{A}}(U, V ; A)= \begin{cases}A \cup V \backslash U, & \text { if } U \subseteq A \subseteq X \backslash V \text { and } A \cup V \backslash U \notin \mathcal{A} \\ A, & \text { otherwise }\end{cases}
$$

and let

$$
\widetilde{T}(U, V ; \mathcal{A})=\left\{T_{\mathcal{A}}(U, V ; A): A \in \mathcal{A}\right\} .
$$

It is not difficult to verify that $\widetilde{T}$ preserves the cardinality of $\mathcal{A}$.

Let $\widetilde{T}(U, V ; \mathcal{A})=\mathcal{A}^{\prime}, \widetilde{T}(U, V ; \mathcal{B})=\mathcal{B}^{\prime}$ and let $A^{\prime}=T_{\mathcal{A}}(U, V ; A)$ and $B^{\prime}=T_{\mathcal{B}}(U, V ; B)$ where $A \in \mathcal{A}$ and $B \in \mathcal{B}$. We claim that $\left|A^{\prime} \cap B^{\prime}\right| \geq t$. If $U=\emptyset$ this is obvious, so we assume $0<|U|<|V|$.

If $A^{\prime} \in \mathcal{A} \cap \mathcal{A}^{\prime}$ and $B^{\prime} \in \mathcal{B} \cap \mathcal{B}^{\prime}$ then the claim is trivially true.

If $A^{\prime} \in \mathcal{A}^{\prime} \backslash \mathcal{A}$ and $B^{\prime} \in \mathcal{B}^{\prime} \backslash \mathcal{B}$, the claim is also obvious.

Without loss of generality, for the remaining possibility assume $A^{\prime} \in \mathcal{A}^{\prime} \backslash \mathcal{A}$ and $B^{\prime} \in \mathcal{B} \cap \mathcal{B}^{\prime}$, so that $B=B^{\prime}$. If $U \subseteq B \subseteq X \backslash V$ then $B \cup V \backslash U \in \mathcal{B}$ and so $\left|A^{\prime} \cap B^{\prime}\right|=|A \cap(B \cup V \backslash U)| \geq t$.

Suppose then that $U \subseteq B \subseteq X \backslash V$ does not hold. Then there are $u \in U$ and $v \in V$ such that not both $u \in B$ and $v \in V \backslash B$ hold. Set

$$
\bar{A}=A \cup(V \backslash\{v\}) \backslash(U \backslash\{u\})=A^{\prime} \cup\{u\} \backslash\{v\} .
$$

Then $|\bar{A}|=\left|A^{\prime}\right|>|A|$ and $d(A, \bar{A})<|U|+|V|=d\left(A_{0}, A_{0}^{\prime}\right)$ so the choice of $A_{0}$ and $A_{0}^{\prime}$ implies that $\bar{A} \in \mathcal{A}$. So

$$
\left|A^{\prime} \cap B^{\prime}\right|=|(\bar{A} \cup\{v\} \backslash\{u\}) \cap B| \geq|\bar{A} \cap B| \geq t .
$$

The first inequality is because of the fact that not both $u \in B$ and $v \in V \backslash B$ hold. This proves the claim. 
Now apply $\widetilde{T}$ to both $\mathcal{A}$ and $\mathcal{B}$ repeatedly with various choices of $U$ and $V$. By the assumption on the sizes of $\mathcal{A}$ and $\mathcal{B}$, we will eventually obtain identical set systems, both isomorphic to $\mathcal{F}(n, t)$. Let $\mathcal{A}^{*}$ and $\mathcal{B}^{*}$ be the set systems before the last step, that is, $\widetilde{T}\left(U, V ; \mathcal{A}^{*}\right)=\mathcal{F}(n, t)$ and $\widetilde{T}\left(U, V ; \mathcal{B}^{*}\right)=\mathcal{F}(n, t)$. So there exists $A \in \mathcal{A}^{*}$ with $|A|<\frac{n+t}{2}$ and $A^{\prime} \notin \mathcal{A}^{*}$ with $\left|A^{\prime}\right|>|A|$.

Case 1. $U \neq \emptyset$

Let $C=X \backslash A$. Let $D$ be a subset of $A$ of size $t-1$ and $D \cap U \neq \emptyset$, and let $B=C \cup D$. It can be easily verified that $B \in \mathcal{F}(n, t)$. But $|A \cap B|=t-1$, so $B \notin \mathcal{B}^{*}$. Since $B \cap U \neq \emptyset, B$ is not produced by $\widetilde{T}\left(U, V ; \mathcal{B}^{*}\right)$. This is a contradiction.

Case 2. $U=\emptyset$

Let $C=X \backslash A$. Let $D$ be a subset of $A$ of size $t-1$, and let $B=C \cup D$. It can be easily verified that $B \in \mathcal{F}(n, t)$. But $|A \cap B|=t-1$, so $B \notin \mathcal{B}^{*}$. So $B$ must be produced by $\widetilde{T}\left(U, V ; \mathcal{B}^{*}\right)$. Let $B_{1}=B \backslash V$. By the above argument, $B_{1} \in \mathcal{B}^{*}$, but then $\left|A \cap B_{1}\right| \leq|A \cap B|=t-1$, a contradiction.

(2) $n+t$ is odd.

If $\mathcal{A}$ or $\mathcal{B}$ is not a Hamming ball then apply the same operation $\widetilde{T}$ in (1) to both $\mathcal{A}$ and $\mathcal{B}$. If $\mathcal{A}$ and $\mathcal{B}$ are both Hamming balls, as shown at the beginning of the proof, they must both be isomorphic to $\mathcal{F}(n, t)$. Without loss of generality, we can assume they are actually equal to $\mathcal{F}(n, t)$. Again let $\mathcal{A}^{*}$ and $\mathcal{B}^{*}$ be the set systems before the last step, that is, $\widetilde{T}\left(U, V ; \mathcal{A}^{*}\right)=$ $\mathcal{F}(n, t)$ and $\widetilde{T}\left(U, V ; \mathcal{B}^{*}\right)=\mathcal{F}(n, t)$.

Let $i$ and $j$ be the smallest integers such that $\mathcal{A}_{i}^{*} \neq \emptyset$ and $\mathcal{B}_{j}^{*} \neq \emptyset$ respectively. Without loss of generality, assume $i \leq j$. Let $A$ be a set in $\mathcal{A}_{i}^{*}$.

If $j \geq i \geq \frac{n+t-1}{2}$ then $\left|\mathcal{A}_{\frac{n+t-1}{2}}^{*}\right| \geq\left(\begin{array}{c}n-1 \\ \frac{n+t-1}{2}\end{array}\right)$ and $\left|\mathcal{B}_{\frac{n+t-1}{2}}^{*}\right| \geq\left(\begin{array}{c}n-1 \\ \frac{n+t-1}{2}\end{array}\right)$, with at least one of the inequalities strict. That contradicts Corollary 2.1.5 (as explained in the beginning of this proof).

So we can assume $i<\frac{n+t-1}{2}$. We let $C=[2, n] \backslash A$, and construct $D$ and $B$ the same way as in (1). By the same argument as in $(1)(U=\emptyset$ or $U \neq \emptyset)$, we get a contradiction. 


\section{Chapter 3}

\section{Sperner $t$-cross-intersecting Set}

\section{Systems}

\subsection{Introduction}

We denote the set of all subsets of the set $X=\{1,2, \cdots, n\}$ by $\mathcal{P}(X)$ and the set of all subsets of $X$ of size $k$ by $X^{(k)}$. We say a set system $\mathcal{F} \subseteq \mathcal{P}(X)$ is a Sperner family if $A \nsubseteq B$ and $B \nsubseteq A$ for each $A, B \in \mathcal{F}$, and we say $\mathcal{F}$ is $t$-intersecting if $|A \cap B| \geq t$ for each $A, B \in \mathcal{F}$ (and that $\mathcal{F}$ is intersecting if it is 1 -intersecting). If $\mathcal{A}, \mathcal{B} \subseteq \mathcal{P}(X)$ we say $\mathcal{A}$ and $\mathcal{B}$ are $t$-cross-intersecting if $|A \cap B| \geq t$ for each $A \in \mathcal{A}$ and $B \in \mathcal{B}$.

A natural generalization of an extremal theorem about $t$-intersecting set systems is a similar theorem about $t$-cross-intersecting set systems $\mathcal{A}$ and $\mathcal{B}$ (where the special case $\mathcal{A}=\mathcal{B}$ reduces to the original theorem). For example, the Erdős-Ko-Rado theorem (Theorem 1A) has several generalizations in this spirit.

Theorem 3.1.1A ([3]). If $k \leq \frac{n}{2}$ and $\mathcal{F} \subseteq X^{(k)}$ is intersecting then $|\mathcal{F}| \leq\left(\begin{array}{c}n-1 \\ k-1\end{array}\right)$.

Kleitman proved the following generalization:

Theorem 3.1.1B ([20]). If $k \leq \frac{n}{2}$ and $\mathcal{A}, \mathcal{B} \subseteq X^{(k)}$ are cross-intersecting then $|\mathcal{A}| \leq\left(\begin{array}{c}n-1 \\ k-1\end{array}\right)$ or $|\mathcal{B}| \leq\left(\begin{array}{c}n-1 \\ k-1\end{array}\right)$. 
Matsumoto and Tokushige [23] strengthened a result of Pyber [27] to obtain the following generalization of Theorem 1B:

Theorem 3.1.1C ([23]). If $\mathcal{A} \subseteq X^{(a)}$ and $\mathcal{B} \subseteq X^{(b)}$ are cross-intersecting where $a \leq \frac{n}{2}$ and $b \leq \frac{n}{2}$ then $|\mathcal{A}||\mathcal{B}| \leq\left(\begin{array}{c}n-1 \\ a-1\end{array}\right)\left(\begin{array}{c}n-1 \\ b-1\end{array}\right)$.

It takes some finesse to obtain a generalization of Theorem 1B in terms of an upper bound on $|\mathcal{A}|+|\mathcal{B}|$, because without extra conditions on $\mathcal{A}$ and $\mathcal{B}$, one can be empty and the other large, and they are still cross-intersecting. Frankl [6] obtained one such generalization.

Theorem 3.1.1D ([6]). If $\mathcal{A}, \mathcal{B} \subseteq X^{(k)}$ are cross-intersecting where $k \leq \frac{n}{2}$ and $|\mathcal{A}| \leq|\mathcal{B}| \leq$ $\left(\begin{array}{l}n-1 \\ k-1\end{array}\right)+\left(\begin{array}{c}n-2 \\ k-2\end{array}\right)$ then $|\mathcal{A}|+|\mathcal{B}| \leq 2\left(\begin{array}{c}n-1 \\ k-1\end{array}\right)$.

There are conditions about when equality holds in each of the four above theorems which we have omitted. Frankl and Tokushige [8], Kisvölcsey [18] and Goldwasser and Ou [10] also found near generalizations of Theorem 1B along the lines of Theorem 1D.

Loosely speaking we say that Theorem 1B is a "one or the other" cross-intersecting generalization of Theorem 1A, and that Theorem 1C and Theorem 1D are "product of cardinalities" and "sum of cardinalities" generalizations of Theorem 1B respectively.

Katona [13] found the maximum size of a $t$-intersecting family $\mathcal{F} \subseteq \mathcal{P}(X)$. Goldwasser and $\mathrm{Ou}[10]$ proved "one or the other" and "sum of cardinalities" $t$-cross-intersecting generalizations, and Matsumoto and Tokushige [24] proved a "product of cardinalities" generalization.

The $g$-shadow $\partial^{(g)}(\mathcal{F})$ of a set system $\mathcal{F} \subseteq X^{(k)}(g \leq k)$ is $\partial^{(g)}(\mathcal{F})=\left\{G \in X^{(g)}: G \subseteq\right.$ $F$ for some $F \in \mathcal{F}\}$; instead of $\partial^{(k-1)}(\mathcal{F})$ we usually say the shadow of $\mathcal{F}$, denoted $\partial(\mathcal{F})$. The relationship between the size of a set system and the size of its shadow is one of the fundamental tools in working with intersecting set systems. If $\mathcal{F} \subseteq X^{(k)}$ is nonempty then the inequality

$$
\frac{|\partial(\mathcal{F})|}{\left(\begin{array}{c}
n \\
k-1
\end{array}\right)} \geq \frac{|\mathcal{F}|}{\left(\begin{array}{l}
n \\
k
\end{array}\right)}
$$

follows easily from considering the bipartite graph with vertex partition $\partial(\mathcal{F}) \cup \mathcal{F}$ and edges showing containment. Since this graph is connected, equality holds if and only if $\mathcal{F}=X^{(k)}$. It follows that if $k>\frac{n}{2}$ then $|\partial(\mathcal{F})| \geq|\mathcal{F}|$. However $\partial(\mathcal{F})$ could be smaller than $\mathcal{F}$ if $k \leq \frac{n}{2}$

(for example if $\mathcal{F}=X^{(k)}$ ). Katona proved that the shadow cannot be smaller if you impose an intersection condition on $\mathcal{F}$ : 
Theorem 3.1.2A ([13]). If $1 \leq g<k, 1 \leq t \leq k, g+t \geq k$ and $\mathcal{F} \subseteq X^{(k)}$ is nonempty and t-intersecting then

$$
\frac{\left|\partial^{(g)}(\mathcal{F})\right|}{\left(\begin{array}{c}
2 k-t \\
g
\end{array}\right)} \geq \frac{|\mathcal{F}|}{\left(\begin{array}{c}
2 k-t \\
k
\end{array}\right)}
$$

with equality if and only if $g+t=k$ and $\mathcal{F}=Y^{(k)}$ for some $Y \in X^{(2 k-t)}$.

Since $g<k$ and $g+k \geq 2 k-t$ it follows that $\left(\begin{array}{c}2 k-t \\ g\end{array}\right) \geq\left(\begin{array}{c}2 k-t \\ k\end{array}\right)$, so $\left|\partial^{(g)}(\mathcal{F})\right| \geq|\mathcal{F}|$ with equality holding if and only if $g+t=k$ and $\mathcal{F}=Y^{(k)}$ for some $Y \in X^{(2 k-t)}$.

This theorem has turned out to be a key ingredient in the proofs of many extremal theorems, including Katona's first proof of the Erdős-Ko-Rado theorem and his proof of the maximum $t$ intersecting set system theorem [13], Milner's proof of the maximum size of a $t$-intersecting Sperner family [26] (Theorem 3A in this chapter), and Frankl's [6] and Goldwasser's [9] proofs of theorems on the maximum size of an intersecting set system with conditions on the degrees.

Frankl proved the following "one or the other" generalization of Theorem 2A:

Theorem 3.1.2B ([4]). Let $g_{1}, g_{2}, k_{1}, k_{2}, t$ be integers satisfying $1 \leq g_{i}<k_{i}, 1 \leq t \leq k_{i}, t+g_{i} \geq$ $k_{i}$ for $i=1,2$. If $\mathcal{F}_{1} \subseteq X^{\left(k_{1}\right)}$ and $\mathcal{F}_{2} \subseteq X^{\left(k_{2}\right)}$ are nonempty and $t$-cross-intersecting then

$$
\frac{\left|\partial^{\left(g_{i}\right)}\left(\mathcal{F}_{i}\right)\right|}{\left(\begin{array}{c}
2 k_{i}-t \\
g_{i}
\end{array}\right)} \geq \frac{\left|\mathcal{F}_{i}\right|}{\left(\begin{array}{c}
2 k_{i}-t \\
k_{i}
\end{array}\right)}
$$

for either $i=1$ or $i=2$. Strict inequality holds for neither $i=1$ nor $i=2$ if and only if $k_{1}=k_{2}=k$ and $\mathcal{F}_{1}=\mathcal{F}_{2}=Y^{(k)}$ for some $Y \in X^{(2 k-t)}$ (in which case equality holds for $i=1$ and $i=2)$.

Again, since $g_{i}<k_{i}$ and $g_{i}+k_{i} \geq 2 k_{i}-t$ it follows that $\left|\partial^{\left(g_{i}\right)}\left(\mathcal{F}_{i}\right)\right| \leq|\mathcal{F}|$ for both $i=1$ and $i=2$ if and only if $k_{1}=k_{2}=k, g_{1}=g_{2}=k-t$ and $\mathcal{F}_{1}=\mathcal{F}_{2}=Y^{(k)}$ for some $Y \in X^{(2 k-t)}$.

Milner found the maximum size of a Sperner $t$-intersecting set system:

Theorem 3.1.3A ([26]). If $\mathcal{F} \subseteq \mathcal{P}(X)$ is Sperner and t-intersecting then $|\mathcal{F}| \leq\left(\begin{array}{c}n \\ \left\lceil\frac{n+t}{2}\right\rceil\end{array}\right)$ with equality if and only if either

(i) $\mathcal{F}=X^{\left(\left\lceil\frac{n+t}{2}\right\rceil\right)}$ or 
(ii) $n+t$ is odd and $\mathcal{F}=\left\{F \in X^{\left(\frac{n+t-1}{2}\right)}: A \subseteq F\right\} \cup\left\{G \in X^{\left(\frac{n+t+1}{2}\right)}: A \nsubseteq G\right\}$ for some fixed $A \in X^{(t)}$.

Milner's proof of this theorem relied on Theorem 2A, while Katona [15] later found a short proof for the special case $t=1$ using a variation of the "cycle method" he used in his second proof of the Erdős-Ko-Rado theorem [14].

Frankl used Theorem 2B (the "one or the other" generalization of Katona's intersecting shadow theorem) to prove a "one or the other" generalization of Theorem 3A:

Theorem 3.1.3B ([4]). If $\mathcal{F}_{1}, \mathcal{F}_{2} \subseteq \mathcal{P}(X)$ are t-cross-intersecting Sperner families then

$$
\left|\mathcal{F}_{i}\right| \leq\left(\begin{array}{c}
n \\
\left\lceil\frac{n+t}{2}\right\rceil
\end{array}\right)
$$

holds for either $i=1$ or $i=2$.

In this chapter we will prove sum and product of cardinality generalizations of Theorem 3B:

Theorem 3.1.4. Let $X=\{1,2, \cdots, n\}$ and let $t \leq\left\lceil\frac{n}{2}\right\rceil$ be a positive integer. We define the function $f_{n, t}(r)$ for integers $r$ in $\left[\frac{n}{2}, \frac{n+t}{2}\right]$ by

$$
f_{n, t}(r)=\left(\begin{array}{l}
n \\
r
\end{array}\right)+\left(\begin{array}{c}
n \\
n+t-r
\end{array}\right)
$$

and we let $F(n, t)=\max _{r}\left\{f_{n, t}(r)\right\}$.

(a) If $\mathcal{A}, \mathcal{B} \subseteq \mathcal{P}(X)$ are t-cross-intersecting Sperner families with $|\mathcal{A}| \geq|\mathcal{B}|$ then $|\mathcal{A}|+|\mathcal{B}| \leq$ $F(n, t)$ with equality if and only if $\mathcal{A}=X^{(r)}$ and $\mathcal{B}=X^{(n+t-r)}$ where $f_{n, t}(r)=F(n, t)$.

(b) Let $p$ be equal to the smallest value of $r$ in $\left[\frac{n}{2}, \frac{n+t}{2}\right]$ such that

$$
\frac{\left(\begin{array}{c}
n+1 \\
r+1
\end{array}\right)}{\left(\begin{array}{c}
n+1 \\
r+1-t
\end{array}\right)} \geq \frac{2 t-(2 r-n+1)}{2 r-n+1}
$$

and be equal to $\frac{n+t}{2}$ if (3.2) is not satisfied for any such $r$. Then $f_{n, t}(p)=F(n, t)$. 
Theorem 3.1.5. Let $X=\{1,2, \cdots, n\}$. For positive integers $t \leq\left\lceil\frac{n}{2}\right\rceil$ and $q \geq 2$ we define the function $f_{n, t, q}(r)$ for integers $r$ in $\left[\frac{n}{2}, \frac{n+t}{2}\right]$ by

$$
f_{n, t, q}(r)=\left(\begin{array}{l}
n \\
r
\end{array}\right)+(q-1)\left(\begin{array}{c}
n \\
n+t-r
\end{array}\right)
$$

and we let $F(n, t, q)=\max _{r}\left\{f_{n, t, q}(r)\right\}$. If $\mathcal{A}_{1}, \mathcal{A}_{2}, \cdots, \mathcal{A}_{q} \subseteq \mathcal{P}(X)$ are pairwise t-cross-intersecting Sperner families with $\left|\mathcal{A}_{1}\right| \geq\left|\mathcal{A}_{i}\right|$ for all $i \in\{2,3, \cdots, q\}$ then

(i) $\sum_{i=1}^{q}\left|\mathcal{A}_{i}\right| \leq F(n, t, q)$ with equality if and only if $\mathcal{A}_{1}=X^{(r)}$ and $\mathcal{A}_{2}=\mathcal{A}_{3}=\cdots=\mathcal{A}_{q}=$ $X^{(n+t-r)}$ where $f_{n, t, q}(r)=F(n, t, q)$

(ii) $\prod_{i=1}^{q}\left|\mathcal{A}_{i}\right| \leq\left(\begin{array}{c}n \\ \left\lfloor\frac{n+t}{2}\right\rfloor\end{array}\right)\left(\begin{array}{c}n \\ \left\lceil\frac{n+t}{2}\right\rceil\end{array}\right)^{q-1}$ with equality if and only if $\mathcal{A}_{1}=X^{\left(\left\lfloor\frac{n+t}{2}\right\rfloor\right)}$ and $\mathcal{A}_{2}=$ $\mathcal{A}_{3}=\cdots=\mathcal{A}_{q}=X^{\left(\left\lceil\frac{n+t}{2}\right\rceil\right)}$.

Since both sides of inequality (3.2) are equal to 1 if $r=\frac{n+t-1}{2}$, if (3.2) is not satisfied for any $r$ then $\frac{n+t}{2}$ must be an integer.

Theorem 3.1.4(a) is obviously just a special case of statement (i) of Theorem 3.1.5, since $f_{n, t}(r)=f_{n, t, 2}(r)$. We give a proof for Theorem 3.1.4(a) in Section 3.2 using the LYM inequality and convexity. While this method could be adapted to prove the sum of cardinalities part of Theorem 3.1.5, in Section 3.3 we use Theorem 2B to prove both the sum and product of cardinalities parts of Theorem 3.1.5. Theorem 3.1.4(b) follows immediately from Proposition 3.4.1 (in Section 3.4), which is just a statement about maximizing the sum of two binomial coefficients. Unfortunately our proof of this is rather long and complicated; we give a sketch of it in Section 3.4.

\subsection{LYM and $t$-cross-intersecting families}

If $\mathcal{F} \subseteq \mathcal{P}(X)$ we let $\mathcal{F}_{k}$ denote $\mathcal{F} \cap X^{(k)}$ for $k \in\{0,1, \cdots, n\}$. The following inequality was proved in $[22,28,25,1]$ and is generally known as the LYM inequality (see [2] for a short proof). 
LYM inequality. Let $\mathcal{F} \subseteq \mathcal{P}(X)$ be a Sperner family and set $f_{k}=\frac{\left|\mathcal{F}_{k}\right|}{\left(\begin{array}{c}n \\ k\end{array}\right)}$ for $k \in\{0,1, \cdots, n\}$. Then $\sum_{k=0}^{n} f_{k} \leq 1$ with equality if and only if $\mathcal{F}=X^{(k)}$ for some $k$.

If $\mathcal{F} \subseteq \mathcal{P}(X)$ we let $\mathcal{F}^{c}$ denote the set of all complements in $X$ of sets in $\mathcal{F}$. If $\mathcal{F} \subseteq X^{(k)}$ we define the upper shadow of $\mathcal{F}$ to be $\partial_{u}(\mathcal{F})=\left\{G \in X^{(k+1)}: G \supseteq F\right.$ for some $\left.F \in \mathcal{F}\right\}$. It is easy to see that $\partial_{u}(\mathcal{F})=\left(\partial\left(\mathcal{F}^{c}\right)\right)^{c}$, so $\left|\partial_{u}(\mathcal{F})\right|=\left|\partial\left(\mathcal{F}^{c}\right)\right|$.

Lemma 3.2.1. Suppose $\mathcal{A}, \mathcal{B} \subseteq \mathcal{P}(X)$ are t-cross-intersecting Sperner families such that $|\mathcal{A}|+|\mathcal{B}|$ is as large as possible. Then $\mathcal{A}_{i}$ and $\mathcal{B}_{i}$ are both empty if $i<\frac{n}{2}$ or $i>\frac{n}{2}+t$.

Proof Let $i$ be the smallest positive integer such that $\mathcal{A}_{i}$ is not empty, and assume $i<\frac{n}{2}$. Then $\left(\mathcal{A}_{i}\right)^{c} \subseteq X^{(n-i)}$ where $n-i>\frac{n}{2}$, so

$$
\left|\partial_{u}\left(\mathcal{A}_{i}\right)\right|=\left|\partial\left(\left(\mathcal{A}_{i}\right)^{c}\right)\right| \geq\left|\left(\mathcal{A}_{i}\right)^{c}\right|=\left|\mathcal{A}_{i}\right|
$$

where the inequality follows from inequality (3.1). If equality holds in (3.3) then by the conditions for equality in (3.1) we must have $\mathcal{A}_{i}=X^{\left(\frac{n-1}{2}\right)}$. Since $\mathcal{A}$ is Sperner, $\mathcal{A}=X^{\left(\frac{n-1}{2}\right)}$ and since $|\mathcal{A}|+$ $|\mathcal{B}|$ is a maximum, $\mathcal{B}=X^{\left(\frac{n+1}{2}+t\right)}$. But this cannot be, because $\mathcal{A}^{\prime}=X^{\left(\frac{n+1}{2}\right)}$ and $\mathcal{B}^{\prime}=X^{\left(\frac{n-1}{2}+t\right)}$ are $t$-cross-intersecting and the sum of their cardinalities is larger. Hence $\left|\partial_{u}\left(\mathcal{A}_{i}\right)\right|>\left|\mathcal{A}_{i}\right|$ and if $\mathcal{A}^{\prime}=\mathcal{A} \backslash \mathcal{A}_{i} \cup \partial_{u}\left(\mathcal{A}_{i}\right)$ then $\left|\mathcal{A}^{\prime}\right|>|\mathcal{A}|, \mathcal{A}^{\prime}$ is Sperner, and $\mathcal{A}^{\prime}$ and $\mathcal{B}$ are $t$-cross-intersecting, a contradiction. Hence $\mathcal{A}_{i}=\emptyset$, and by the same argument $\mathcal{B}_{i}=\emptyset$, if $i<\frac{n}{2}$.

Let $j$ be the maximum integer such that $\mathcal{A}_{j} \neq \emptyset$. If $j>\frac{n}{2}+t$ then we set $\mathcal{A}^{\prime}=\left(\mathcal{A} \backslash \mathcal{A}_{j}\right) \cup$ $\partial\left(\mathcal{A}_{j}\right)$. Clearly $\mathcal{A}^{\prime}$ is Sperner and $\left|\mathcal{A}^{\prime}\right|>|\mathcal{A}|$. Since $\mathcal{B}_{i}=\emptyset$ for $i<\frac{n}{2}, \mathcal{A}^{\prime}$ and $\mathcal{B}$ are $t$-crossintersecting, a contradiction. By the same argument, $\mathcal{B}=\emptyset$ if $j>\frac{n}{2}+t$.

Proof of Theorem 3.1.4(a) Assume $\mathcal{A}$ and $\mathcal{B}$ are Sperner $t$-cross-intersecting families with $|\mathcal{A}| \geq|\mathcal{B}|$ and such that $|\mathcal{A}|+|\mathcal{B}|$ is a maximum. Let $\mathcal{A}_{i}=\mathcal{A} \cap X^{(i)}$ and $a_{i}=\frac{\left|\mathcal{A}_{i}\right|}{\left(\begin{array}{c}n \\ i\end{array}\right)}$ for $i=\{0,1, \cdots, n\}$ and similarly define $\mathcal{B}_{i}$ and $b_{i}$. Set $m=\left\lfloor\frac{n}{2}\right\rfloor+t$. Since $\mathcal{A}$ and $\mathcal{B}^{c}$ are each 
Sperner and $\mathcal{A}$ and $\mathcal{B}$ are $t$-cross-intersecting, we claim the set system

$$
\left[\bigcup_{i=\left\lceil\frac{n}{2}\right\rceil}^{k} \mathcal{A}_{i}\right] \bigcup\left[\bigcup_{j=\left\lceil\frac{n}{2}\right\rceil}^{n+t-1-k}\left(\mathcal{B}_{j}\right)^{c}\right]
$$

is Sperner for each $k \in\left[\left\lceil\frac{n}{2}\right\rceil, m-1\right]$. This is so because if $A \in \mathcal{A}_{i}$ and $B \in \mathcal{B}_{j}$ then $B^{c}$ contains at least $n+t-i-j \geq 1$ elements not in $A$, and $A$ contains at least $t$ elements not in $B^{c}$. Since $\frac{\left|\left(\mathcal{B}_{j}\right)^{c}\right|}{\left(\begin{array}{c}n \\ n-j\end{array}\right)}=b_{j}$, by the LYM inequality,

$$
\sum_{i=\left\lceil\frac{n}{2}\right\rceil}^{k} a_{i}+\sum_{j=\left\lceil\frac{n}{2}\right\rceil}^{n+t-1-k} b_{j} \leq 1
$$

for each $k \in\left[\left\lceil\frac{n}{2}\right\rceil-1, m-1\right]$, where an empty sum is 0 .

By Lemma 3.2.1 we may assume $a_{i}=b_{i}=0$ if $i<\left\lceil\frac{n}{2}\right\rceil$ or $i>m$. We let $a_{i}^{\prime}=a_{i}$ for $i \in\left[\left\lceil\frac{n}{2}\right\rceil, m-1\right]$ and $a_{m}^{\prime}=1-\sum_{i=\left\lceil\frac{n}{2}\right\rceil}^{m-1} a_{i}$. Hence $\sum_{i=\left\lceil\frac{n}{2}\right\rceil}^{m} a_{i}^{\prime}=1$ and by $(3.4)$ :

$$
\begin{aligned}
0 & \leq \sum_{i=\left\lceil\frac{n}{2}\right\rceil}^{m} a_{i}^{\prime}-\left[\sum_{i=\left\lceil\frac{n}{2}\right\rceil}^{k} a_{i}^{\prime}+\sum_{j=\left\lceil\frac{n}{2}\right\rceil}^{n+t-1-k} b_{j}\right] \\
& =\sum_{i=k+1}^{m} a_{i}^{\prime}-\sum_{j=\left\lceil\frac{n}{2}\right\rceil}^{n+t-1-k} b_{j} \\
& =\sum_{j=\left\lceil\frac{n}{2}\right\rceil}^{n+t-1-k}\left[a_{n+t-j}^{\prime}-b_{j}\right] .
\end{aligned}
$$

Thus

$$
\sum_{i=\left\lceil\frac{n}{2}\right\rceil}^{s}\left(a_{n+t-i}^{\prime}-b_{i}\right) \geq 0
$$


for each $s \in\left[\left\lceil\frac{n}{2}\right\rceil, m\right]$. Now let $w_{i}=\left(\begin{array}{c}n \\ i\end{array}\right)$ for integers $i \in\left[\left\lceil\frac{n}{2}\right\rceil, m\right]$ and let $w_{m+1}=0$. Clearly $w_{\left\lceil\frac{n}{2}\right\rceil}, w_{\left\lceil\frac{n}{2}\right\rceil+1}, \cdots, w_{m}, w_{m+1}$ is a strictly decreasing sequence. Then

$$
\sum_{i=\left\lceil\frac{n}{2}\right\rceil}^{m}\left(w_{i} a_{i}^{\prime}+w_{i} b_{i}\right)=\sum_{i=\left\lceil\frac{n}{2}\right\rceil}^{m}\left[a_{i}^{\prime}\left(w_{i}+w_{n+t-i}\right)-w_{i}\left(a_{n+t-i}^{\prime}-b_{i}\right)\right]
$$

where

$$
\begin{aligned}
\sum_{i=\left\lceil\frac{n}{2}\right\rceil}^{m} w_{i}\left(a_{n+t-i}^{\prime}-b_{i}\right) & =\sum_{i=\left\lceil\frac{n}{2}\right\rceil}^{m} \sum_{s=i}^{m}\left(w_{s}-w_{s+1}\right)\left(a_{n+t-i}^{\prime}-b_{i}\right) \\
& =\sum_{s=\left\lceil\frac{n}{2}\right\rceil}^{m}\left(w_{s}-w_{s+1}\right) \sum_{i=\left\lceil\frac{n}{2}\right\rceil}^{s}\left(a_{n+t-i}^{\prime}-b_{i}\right) \\
& \geq 0
\end{aligned}
$$

the inequality following from (3.5). Hence

$$
\begin{aligned}
|\mathcal{A}|+|\mathcal{B}| & =\sum_{i=\left\lceil\frac{n}{2}\right\rceil}^{m}\left(w_{i} a_{i}+w_{i} b_{i}\right) \\
& \leq \sum_{i=\left\lceil\frac{n}{2}\right\rceil}^{m}\left(w_{i} a_{i}^{\prime}+w_{i} b_{i}\right) \\
& \leq \sum_{i=\left\lceil\frac{n}{2}\right\rceil}^{m} a_{i}^{\prime}\left(w_{i}+w_{n+t-i}\right) \\
& =\sum_{i=\left\lceil\frac{n}{2}\right\rceil}^{m} a_{i}^{\prime} f_{n, t}(i)
\end{aligned}
$$

the second inequality following from (3.6). We have shown that $|\mathcal{A}|+|\mathcal{B}|$ is equal to at most a convex combination of $f_{n, t}(i)$ for various values of $i$. Hence $|\mathcal{A}|+|\mathcal{B}| \leq F(n, t)$.

Equality can hold in the theorem only if it holds in (3.5) for each $s$ and $a_{m}^{\prime}=a_{m}$. That 
means $\sum_{i=\left\lceil\frac{n}{2}\right\rceil}^{m} a_{i}=1$, so by LYM $a_{r}=1$ for some $r$. With equality holding in (3.5), it follows that $b_{n+t-r}=1$, so $\mathcal{A}=X^{(r)}$ and $\mathcal{B}=X^{(n+t-r)}$ where $f_{n, t}(r)=F(n, t)$.

We remark that monotonicity of the sequence $\left\{w_{i}\right\}$ (not the fact that they are binomial coefficients) is the property needed to prove inequality (3.6), and hence the reason that $|\mathcal{A}|+|\mathcal{B}|=$ $\sum_{i=\left\lceil\frac{n}{2}\right\rceil}^{m}\left(w_{i} a_{i}+w_{i} b_{i}\right)$ is maximized by a convex combination of $\left\{w_{i}+w_{n+t-i}\right\}$.

\subsection{Proof of Theorem 3.1.5}

Theorem 3.1.5 follows easily from the following proposition.

Proposition 3.3.1. Suppose $\mathcal{A}_{1} \subseteq X^{\left(p_{1}\right)}$ and $\mathcal{A}_{2} \subseteq X^{\left(p_{2}\right)}$ are nonempty and t-cross-intersecting where $p_{1}+p_{2}<n+t$. Then $\left|\partial_{u}\left(\mathcal{A}_{i}\right)\right|>\left|\mathcal{A}_{i}\right|$ for $i=1$ or $i=2$ or else $p_{1}=p_{2}=p=\frac{n+t-1}{2}$ and $\mathcal{A}_{1}=\mathcal{A}_{2}=\left\{A \in X^{(p)}: A \supseteq C\right\}$ for some $C \in X^{(t)}$. In this last case, $\left|\mathcal{A}_{1}\right|=\left|\mathcal{A}_{2}\right|=\left|\partial_{u}\left(\mathcal{A}_{1}\right)\right|=$ $\left|\partial_{u}\left(\mathcal{A}_{2}\right)\right|=\left(\begin{array}{l}n-t \\ p-t\end{array}\right)$.

Proof We apply Theorem 2B to the set systems $\left(\mathcal{A}_{1}\right)^{c} \subseteq X^{\left(n-p_{1}\right)}$ and $\left(\mathcal{A}_{2}\right)^{c} \subseteq X^{\left(n-p_{2}\right)}$, which are $\left(n+t-p_{1}-p_{2}\right)$-cross-intersecting. We still have $k_{i}=n-p_{i}$ and $g_{i}=k_{i}-1$ for $i=1,2$, and it is easy to check the hypotheses are satisfied. By the comment following Theorem $2 \mathrm{~B}$ it follows that $\left|\partial\left(\left(\mathcal{A}_{i}\right)^{c}\right)\right|>\left|\left(\mathcal{A}_{i}\right)^{c}\right|$ for $i=1$ or $i=2$ or else $k_{1}=k_{2}=k, g_{1}=g_{2}=k-\left(n+t-p_{1}-p_{2}\right)=n-k-t$, and $\left(\mathcal{A}_{1}\right)^{c}=\left(\mathcal{A}_{2}\right)^{c}=Y^{(k)}$ for some $Y \in X^{(n-t)}$. Since $g_{1}=g_{2}=n-k-t=k-1$, we must have $k=\frac{n-t+1}{2}$. Hence $p_{1}=p_{2}=p=\frac{n+t-1}{2}$ and $\mathcal{A}_{1}=\mathcal{A}_{2}=\left\{A \in X^{(p)}: A \supseteq Y^{c}\right\}$. The last statement in the proposition is easy to check.

Proof of Theorem 3.1.5 Suppose $\mathcal{A}_{1}, \mathcal{A}_{2}, \cdots, \mathcal{A}_{q} \subseteq \mathcal{P}(X)$ are pairwise $t$-cross-intersecting Sperner families with $\left|\mathcal{A}_{1}\right| \geq\left|\mathcal{A}_{i}\right|$ for $i \in\{2,3, \cdots, q\}$. For $i \in\{1,2, \cdots, q\}$ and $j \in\{0,1, \cdots, n\}$ we let $\mathcal{A}_{i j}=\mathcal{A}_{i} \cap X^{(j)}$. Let $p$ be the smallest value of $j$ such that $\mathcal{A}_{i j}$ is nonempty for some $i \in\{1,2, \cdots, q\}$, and let $k \in\{1,2, \cdots, q\}$ be such that $\mathcal{A}_{k p} \neq \emptyset$. Let $s$ be the smallest value of $j$ such that $\mathcal{A}_{m j}$ is nonempty for some $m \neq k$ and let $h \in\{1,2, \cdots, q\} \backslash\{k\}$ be such that $\mathcal{A}_{h s} \neq \emptyset$. So if $\mathcal{A}_{b c}$ and $\mathcal{A}_{d e}$ are nonempty with $b \neq d$ then $c+e \geq p+s$. 
We claim that if $p+s<n+t$ then either $\mathcal{A}_{k}$ or $\mathcal{A}_{h}$ can be replaced by a larger Sperner family, which along with the other original $q-1$ families will still be pairwise $t$-cross-intersecting. This is obviously the case if $\left|\partial_{u}\left(\mathcal{A}_{k p}\right)\right|>\left|\mathcal{A}_{k p}\right|$ or $\left|\partial_{u}\left(\mathcal{A}_{h s}\right)\right|>\left|\mathcal{A}_{h s}\right|$. If neither of these inequalities holds then, by Proposition 3.3.1, $p=s=\frac{n+t-1}{2}, \mathcal{A}_{k p}=\mathcal{A}_{h s}=\left\{A \in X^{(p)}: A \supseteq C\right\}$ for some $C \in X^{(t)}$, and $\left|\mathcal{A}_{k p}\right|=\left|\mathcal{A}_{h s}\right|=\left|\partial_{u}\left(\mathcal{A}_{k p}\right)\right|=\left|\partial_{u}\left(\mathcal{A}_{h s}\right)\right|=\left(\begin{array}{c}n-t \\ p-t\end{array}\right)$. So we can replace $\mathcal{A}_{k p}$ (or $\mathcal{A}_{h s}$ or both) by its upper shadow, and we have the same size set system. But $\partial_{u}\left(\mathcal{A}_{k p}\right)$ is a proper subset of $X^{(p+1)}$. Since $(p+1)+s=n+t$ and $p+1 \geq \frac{n}{2}$, by the LYM inequality $\left|\mathcal{A}_{k}\right|<\left(\begin{array}{c}n \\ p+1\end{array}\right)$,

so we can replace $\mathcal{A}_{k}$ with $X^{(p+1)}$ and still have $q$ pairwise $t$-cross-intersecting Sperner families, completing the proof of the claim.

So if $\sum\left|\mathcal{A}_{i}\right|$ (or $\prod\left|\mathcal{A}_{i}\right|$ ) is a maximum then if $\mathcal{A}_{g w} \neq \emptyset$ for some $g \neq k$ we must have $w \geq n+t-p$. Now let $f$ be the largest integer $j$ such that $\mathcal{A}_{g j} \neq \emptyset$ for some $g \neq k$ and let

$y \in\{1,2, \cdots, q\} \backslash\{k\}$ be such that $\mathcal{A}_{y f} \neq \emptyset$. Suppose $f>n+t-p$. Then $f \geq \frac{f+p}{2}>\frac{n+t}{2}$, so $\left|\partial\left(\mathcal{A}_{y f}\right)\right|>\left|\mathcal{A}_{y f}\right|$. That means we can replace $\mathcal{A}_{y f}$ with $\partial\left(\mathcal{A}_{y f}\right)$ in $\mathcal{A}_{y}$ and get a bigger set, which along with the other $q-1$ families will still be $t$-cross-intersecting. Hence if $\sum\left|\mathcal{A}_{i}\right|$ (or $\left.\prod\left|\mathcal{A}_{i}\right|\right)$ is a maximum, then $\mathcal{A}_{i}=X^{(n+t-p)}$ for all $i \neq k$. By the same reasoning used to prove Lemma 3.2.1, $p \geq \frac{n}{2}$, and by LYM again, $\mathcal{A}_{k}=X^{(p)}$. So our only candidates to maximize $\sum\left|\mathcal{A}_{i}\right|$

and $\prod\left|\mathcal{A}_{i}\right|$ are $\mathcal{A}_{1}=X^{(r)}, \mathcal{A}_{2}=\mathcal{A}_{3}=\cdots=\mathcal{A}_{q}=X^{(n+t-r)}$ for some $r \in\left[\left\lceil\frac{n}{2}\right\rceil,\left\lfloor\frac{n+t}{2}\right\rfloor\right]$. That completes the proof of (i).

The statement in (ii) follows because if $\frac{n}{2} \leq r \leq d-2 \leq n-1$ then

$$
\frac{\left(\begin{array}{c}
n \\
r+1
\end{array}\right)\left(\begin{array}{c}
n \\
d-1
\end{array}\right)}{\left(\begin{array}{c}
n \\
r
\end{array}\right)\left(\begin{array}{c}
n \\
d
\end{array}\right)}=\frac{n-r}{n-(d-1)} \cdot \frac{d}{r+1}>1
$$

so the product is largest when $r$ is as big as possible.

We remark that Theorem 3B and Theorem 3A only follow immediately from the sum and product of cardinality generalizations in Theorem 3.1.5 if $n+t$ is even. When $n+t$ is odd, since one of the families in an optimal configuration is bigger than the others, you need to impose additional conditions. If you make the assumption that all families in an optimal configuration have the same size, then you can modify the proof of Theorem 3.1.5 to get a theorem which gives Theorem 3B and Theorem 3A as special cases. The third possibility in Proposition 3.3.1 is what leads to the different optimal configurations in Theorem 3A. 


\subsection{Sums of binomial coefficients}

To complete the proof of Theorem 3.1.4(b) it suffices to prove the following proposition about binomial coefficients:

Proposition 3.4.1. For positive integers $n$ and $t$ such that $t \leq n-1$ we let $f(r)=\left(\begin{array}{c}n \\ r\end{array}\right)+\left(\begin{array}{c}n \\ n+t-r\end{array}\right)$ for integers $r \in\left[\frac{n}{2}, \frac{n+t}{2}\right]$. Then $f(r)$ is unimodal. That is, there is an integer $m \in\left[\frac{n}{2}, \frac{n+t}{2}\right]$ such that $f(r)$ is increasing for $r \in\left[\frac{n}{2}, m\right]$ and decreasing for $r \in\left[m, \frac{n+t}{2}\right]$. The integer $m$ is equal to the smallest value of $r$ such that

$$
\frac{\left(\begin{array}{c}
n+1 \\
r+1
\end{array}\right)}{\left(\begin{array}{c}
n+1 \\
r+1-t
\end{array}\right)} \geq \frac{2 t-(2 r-n+1)}{2 r-n+1}
$$

and is equal to $\left\lfloor\frac{n+t}{2}\right\rfloor$ if (3.7) is not satisfied for any $r$.

Proof For $r \in\left[\frac{n}{2}, \frac{n+t}{2}-1\right]$ we let $\triangle f(r)=f(r+1)-f(r)$. So

$$
\begin{aligned}
\triangle f(r) & =\left[\left(\begin{array}{c}
n \\
r-t+1
\end{array}\right)-\left(\begin{array}{c}
n \\
r-t
\end{array}\right)\right]-\left[\left(\begin{array}{l}
n \\
r
\end{array}\right)-\left(\begin{array}{c}
n \\
r+1
\end{array}\right)\right] \\
& =\frac{2 r-n+1}{n+1}\left(\begin{array}{l}
n+1 \\
r+1
\end{array}\right)\left[\frac{\left(\begin{array}{c}
n+1 \\
r+1-t
\end{array}\right)}{\left(\begin{array}{l}
n+1 \\
r+1
\end{array}\right)} \cdot \frac{2 t-(2 r-n+1)}{2 r-n+1}-1\right]
\end{aligned}
$$

and (3.7) is satisfied if and only if $\triangle f(r) \leq 0$. If we show $\triangle f(r+1) \geq 0 \Longrightarrow \triangle f(r) \geq 0$ for all $r \in\left[\frac{n}{2}, \frac{n+t}{2}-2\right]$, it follows that $f(r)$ is unimodal, with $f(r)$ taking on its maximum value at $r=m$, where $m$ is the smallest value of $r$ such that $\triangle f(r) \leq 0$, and $m=\left\lceil\frac{n+t}{2}\right\rceil$ if $\triangle f(r)>0$ for all $r$.

We define the function $G(r)$ by

$$
G(r)=\frac{\left(\begin{array}{c}
n+1 \\
r+1-t
\end{array}\right)}{\left(\begin{array}{c}
n+1 \\
r+1
\end{array}\right)} \cdot \frac{2 t-(2 r-n+1)}{2 r-n+1}
$$


for $r \in\left[\frac{n}{2}, \frac{n+t}{2}-1\right]$. For $r \in\left[\frac{n}{2}, \frac{n+2}{2}-2\right]$ we have

$$
\begin{aligned}
\frac{G(r)}{G(r+1)} & =\frac{n-r}{n+t-r} \cdot \frac{r+2-t}{r+2} \cdot \frac{2 r-n+3}{2 r-n+1} \cdot \frac{2 t-1+n-2 r}{2 t-3+n-2 r} \\
& =\frac{H(r)+2 n-n t}{H(r)+2 n+2 t} \cdot \frac{4 H(r)-(n-1+2 t)(n-3)}{4 H(r)-(n-1+2 t)(n-3)-4 t}
\end{aligned}
$$

where $H(r)=-r^{2}+r(n+t-2)$. Now assume $\frac{G(r)}{G(r+1)} \geq 1$ for some $r$ in $\left[\frac{n}{2}+1, \frac{n+t}{2}-2\right]$. We will show this implies that $\frac{G(r-1)}{G(r)}>1$. Letting $A=H(r)+2 n+2 t$ and $B=H(r)-\frac{(n-1+2 t)(n-3)}{4}$ we have

$$
\frac{G(r)}{G(r+1)}=\frac{A-(n+2) t}{A} \cdot \frac{B}{B-t}
$$

and

$$
\begin{aligned}
H(r-1) & =-(r-1)^{2}+(r-1)(n+t-2) \\
& =H(r)-(n+t-1-2 r) \\
& =H(r)-w
\end{aligned}
$$

where $w=n+t-1-2 r$. Now it follows from (3.8) and (3.9) that

$$
\begin{aligned}
\frac{G(r-1)}{G(r)} & =\frac{A-(n+2) t-w}{A-w} \cdot \frac{B-w}{B-t-w} \\
& =\frac{(A-(n+2) t) B-w(A+B-w)+(n+2) t w}{A(B-t)-w(A+B-w)+t w}>1
\end{aligned}
$$

the inequality following because $\frac{G(r)}{G(r+1)} \geq 1$.

We have shown that $G(r)>G(r+1) \Longrightarrow G(r-1)>G(r)$. Hence there exists an integer $m^{\prime}$ such that $G(r)$ is decreasing for $r \leq m^{\prime}$ and increasing for $r \geq m^{\prime}$. If $G(r)$ is decreasing for all $r$, there is nothing to prove (since then $\triangle f(r)$ cannot switch from negative to positive as $r$ increases). If not, but if $G\left(\left\lfloor\frac{n+t}{2}\right\rfloor-1\right) \leq 1$, there is still nothing to prove (since $\triangle f(r)$ still cannot switch from negative to positive as $r$ increases). So to finish the proof it suffices to show that if $G\left(\left\lfloor\frac{n+t}{2}\right\rfloor-1\right)>1$ then $G\left(\left\lfloor\frac{n+t}{2}\right\rfloor-2\right)>G\left(\left\lfloor\frac{n+t}{2}\right\rfloor-1\right)$. 
If $n+t$ is even we have $G\left(\frac{n+t}{2}-1\right)=\frac{n-t+2}{n+t+2} \cdot \frac{t+1}{t-2}$ and

$$
\begin{aligned}
& G\left(\frac{n+t}{2}-2\right) \\
= & G\left(\frac{n+t}{2}-1\right) \cdot \frac{\frac{n-t}{2}\left(\frac{n-t}{2}+2\right)}{\frac{n+t}{2}\left(\frac{n+t}{2}+2\right)} \cdot \frac{t-1}{t+1} \cdot \frac{t+3}{t-3} \\
= & G\left(\frac{n+t}{2}-1\right) \cdot \frac{(n-t+2)^{2}-4}{(n+t+2)^{2}-4} \cdot \frac{(t+1)^{2}-4}{(t-1)^{2}-4} \\
= & G\left(\frac{n+t}{2}-1\right) \cdot \frac{[(n-t+2)(t+1)]^{2}-4\left[(n+2)^{2}-3+2 t^{2}\right]+8 t(n+1)}{[(n+t+2)(t-1)]^{2}-4\left[(n+2)^{2}-3+2 t^{2}\right]-8 t(n+1)} \\
> & G\left(\frac{n+t}{2}-1\right)
\end{aligned}
$$

the inequality following because $\left[\frac{(n-t+2)(t+1)}{(n+t+2)(t-1)}\right]^{2}=G\left(\frac{n+t}{2}-1\right)^{2}>1$.

A similar (slightly more complicated) calculation when $n+t$ is odd shows that if $G\left(\frac{n+t-3}{2}\right)>$ 1 then $G\left(\frac{n+t-5}{2}\right)>G\left(\frac{n+t-3}{2}\right)$.

It is undoubtedly possible to find a condition analogous to inequality (3.2) in Theorem 3.1.4 to determine which value of $r$ maximizes $f_{n, t, q}(r)$. In view of the complications even when $q=2$ we decided not to look into this.

For fixed $t$, for which values of $n$ is $f_{n, t}(r)$ monotonic? The following proposition answers that question.

Proposition 3.4.2. For each $t \geq 2$ let $m_{0}(t)$ be the largest value of $n$ such that $f\left(\left\lceil\frac{n}{2}\right\rceil\right)=F(n, t)$ and let $n_{0}(t)$ be the smallest value of $n$ such that $f\left(\left\lfloor\frac{n+t}{2}\right\rfloor\right)=F(n, t)$. Then

(a) there exists a positive integer $t_{0}$ such that for all positive real numbers $c_{1}, c_{2}, \epsilon$,

$$
c_{1} t^{2-\epsilon}<m_{0}(t)<c_{2} t^{2}
$$

for all $t \geq t_{0}$. 
(b) $n_{0}(t)=t^{2}-3$.

The statement in (b) follows from the fact that $G\left(\frac{n+t-3}{2}\right)=G\left(\frac{n+t-1}{2}\right)=1$ when $n=t^{2}-3$. To prove (a) we used Riemann sums to show that for all positive integers $p$ and $q$ with $q<p$,

$$
\frac{(p+q)^{p+q}(p-q)^{p-q}}{p^{2 p}}<\frac{(p+1)(p+2) \cdots(p+q)}{(p-1)(p-2) \cdots(p-q)}<\frac{(p+q)^{p+q}(p-q)^{p-q}}{p^{2 p}} \cdot \frac{p+q}{p-q}
$$

Letting $p=\frac{n}{2}+1$ and $q=t-1$ gives an estimate for $G\left(\left\lceil\frac{n}{2}\right\rceil\right)$. We omit the details. In fact we have shown that $m_{o}(t)=\Theta\left(\frac{t^{2}}{\log t}\right)$. 


\section{Chapter 4}

\section{Intersecting Set Systems with}

\section{Degree Conditions}

\subsection{Notation and Background}

In this chapter we use the following notation.

Let $\left[n_{1}, n_{2}\right]=\left\{n_{1}, n_{1}+1, n_{1}+2, \cdots, n_{2}\right\},[n]=[1, n]$.

Let $X$ be a finite set and $\mathcal{P}(X)$ be the power set of $X$. Define $X^{(k)}=\{F \subseteq X:|F|=k\}$.

Let $\mathcal{F} \subseteq \mathcal{P}(X)$ be a set system, $i, j \in X$. Define $\mathcal{F}_{i}=\{F \in \mathcal{F}: i \in F\}, \mathcal{F}_{\bar{i}}=\{F \in \mathcal{F}$ : $i \notin F\}, \mathcal{F}(i)=\{F \backslash\{i\}: i \in F\} . \mathcal{F}_{i, j}=\mathcal{F}_{i} \cap \mathcal{F}_{j}$. Define $\mathcal{F}_{i, \bar{j}}$ etc. similarly. The degree of $i$, denoted by $d_{\mathcal{F}}(i)$, is the number of subsets in $\mathcal{F}$ that contain $i$, i.e., $d_{\mathcal{F}}(i)=\left|\mathcal{F}_{i}\right|=|\mathcal{F}(i)|$. The complementary degree of $i$, denoted by $c_{\mathcal{F}}(i)$, is defined to be $|\mathcal{F}|-d_{\mathcal{F}}(i)$. Sometimes we omit the subscript $\mathcal{F}$ if it is clear from the context. Define $d(\mathcal{F})=\max _{i \in \mathcal{F}} d(i), c(\mathcal{F})=\min _{i \in \mathcal{F}} c(i)$.

Let $\mathcal{F} \subseteq X^{(k)}, l<k$, the $l$-shadow of $\mathcal{F}$, denoted by $\partial^{(l)} \mathcal{F}$, is defined to be $\left\{A \in X^{(l)}: A \subseteq\right.$ $F$ for some $F \in \mathcal{F}\}$.

$$
\partial^{(l)}(\mathcal{F}, i)=\left\{A \in \partial^{(l)} \mathcal{F}: i \in A\right\}
$$


$\partial^{(l)}(\mathcal{F}, \bar{i})=\left\{A \in \partial^{(l)} \mathcal{F}: i \notin A\right\}$

$\partial^{(l)}(\mathcal{F}, i \wedge j)=\left\{A \in \partial^{(l)} \mathcal{F}: i \in A\right.$ and $\left.j \in A\right\}$

$\partial^{(l)}(\mathcal{F}, i \vee j)=\left\{A \in \partial^{(l)} \mathcal{F}: i \in A\right.$ or $\left.j \in A\right\}$

Similarly define $\partial^{(l)}(\mathcal{F}, i \wedge \bar{j})$.

For $S \subseteq X$, define $\partial^{(l)}(\mathcal{F}, S)=\left\{A \in \partial^{(l)} \mathcal{F}: S \subseteq A\right\}$

Let $\mathcal{F} \subseteq X^{(k)}, A \in \mathcal{F}$, " $\leq$ " be a linear order on $\mathcal{F}$. Unless otherwise specified, take " $\leq$ " to be the COLEX order, which is defined in previous chapters.

$$
\begin{aligned}
& \operatorname{mem}(\leq, \mathcal{F}, m)=\text { the } m \text {-th member of } \mathcal{F} \text { with respect to the order " } \leq \text { ". } \\
& \operatorname{ind}(\leq, \mathcal{F}, A)=\text { the index of } A \text { in } \mathcal{F} \text { with respect to the order " } \leq " \\
& \mathcal{L}(\leq, \mathcal{F}, m)=\{F \in \mathcal{F}: \operatorname{ind}(F) \leq m\} \\
& \mathcal{L}(\leq, \mathcal{F}, A)=\{F \in \mathcal{F}: F \leq A\} .
\end{aligned}
$$

Sometimes we omit " $\leq$ " if it is clear from the context which order to use.

The following notation is taken from [2]. For $0 \leq m_{s}<m_{s+1}<\cdots<m_{r}$ let

$$
\mathcal{B}^{(k)}\left(m_{k}, \cdots, m_{s}\right)=\cup_{j=s}^{r}\left(\left[m_{j}\right]^{(j)}+\left\{m_{j+1}+1, \cdots, m_{r}+1\right\}\right)
$$

and

$$
b^{(k)}\left(m_{k}, \cdots, m_{s}\right)=\sum_{j=s}^{r}\left(\begin{array}{c}
m_{j} \\
j
\end{array}\right) .
$$

It can be shown that $\left|\mathcal{B}^{(k)}\left(m_{k}, \cdots, m_{s}\right)\right|=b^{(k)}\left(m_{k}, \cdots, m_{s}\right)$.

Let $t=\left(\begin{array}{c}m_{k} \\ k\end{array}\right)+\left(\begin{array}{c}m_{k-1} \\ k-1\end{array}\right)+\cdots+\left(\begin{array}{c}m_{h} \\ h\end{array}\right)$ be the cascade form of $t$. Define len $(k, t)=k-h+1$, the number of terms in the cascade form. Define $\operatorname{len}(k, A)=\operatorname{len}(k, \operatorname{ind}(\leq, \mathcal{F}, A))$. Define $P(k, t, s)=\left(\begin{array}{c}m_{k} \\ k\end{array}\right)+\left(\begin{array}{c}m_{k-1} \\ k-1\end{array}\right)+\cdots+\left(\begin{array}{c}m_{s} \\ s\end{array}\right), R(k, t, s)=t-P(k, t, s)$ for $s \geq h$. As a convention, if $s<h$, define $P(k, t, s)=t, R(k, t, s)=0$; if $s>k$, define $P(k, t, s)=0, R(k, t, s)=t$. Define $P(k, A, s)=P(k, \operatorname{ind}(\leq, \mathcal{F}, A), s)$.

Define

$$
C(k, t, s)= \begin{cases}P(k, t, s)+\left(\begin{array}{c}
m_{s} \\
s-1
\end{array}\right), & \text { if } t>P(k, t, s) \\
P(k, t, s), & \text { otherwise }\end{cases}
$$


Let $x$ be a symbol not in any member of $\mathcal{F}$, define $\mathcal{F}+\{x\}=\{A \cup\{x\}: A \in \mathcal{F}\}$.

Let $\mathcal{A}, \mathcal{B}$ be two set systems, $\mathcal{A}_{0} \subseteq \mathcal{A}$. Let $a=|\mathcal{A}|, b=|\mathcal{B}|, a_{0}=\left|\mathcal{A}_{0}\right|$.

Define $a^{\prime}=\min \left\{a_{0}+b, a\right\}, b^{\prime}=a_{0}+b-a^{\prime}$, and define $\mathcal{R}_{1}\left(\mathcal{A}, \mathcal{A}_{0}, \mathcal{B}\right)=\mathcal{L}\left(\leq, \mathcal{A}, a^{\prime}\right)$, $\mathcal{R}_{2}\left(\mathcal{A}, \mathcal{A}_{0}, \mathcal{B}\right)=\mathcal{L}\left(\leq, \mathcal{B}, b^{\prime}\right)$.

Let $\mathcal{A}_{0}, \mathcal{B} \subseteq \mathcal{F}, \mathcal{A}_{0} \subseteq \mathcal{A}, \mathcal{A} \cap \mathcal{B}=\emptyset$. Define $\mathcal{R}\left(\mathcal{F}, \mathcal{A}, \mathcal{A}_{0}, \mathcal{B}\right)=\mathcal{F} \backslash\left(\mathcal{A}_{0} \cup \mathcal{B}\right) \cup \mathcal{R}_{1}\left(\mathcal{A}, \mathcal{A}_{0}, \mathcal{B}\right) \cup$ $\mathcal{R}_{2}\left(\mathcal{A}, \mathcal{A}_{0}, \mathcal{B}\right)$.

Theorem 4.1.1. Let $\mathcal{A}=X^{\left(k_{1}\right)}, \mathcal{B}=X^{\left(k_{2}\right)}$ with $k_{1} \leq k_{2}, \mathcal{A}_{0} \subseteq \mathcal{A}, \mathcal{B}_{0} \subseteq \mathcal{B}$. Let $\mathcal{A}^{\prime}=$ $\mathcal{R}_{1}\left(\mathcal{A}, \mathcal{A}_{0}, \mathcal{B}\right), \mathcal{B}^{\prime}=\mathcal{R}_{2}\left(\mathcal{A}, \mathcal{A}_{0}, \mathcal{B}\right)$. Then $\left|\partial^{\left(k_{1}-d\right)} \mathcal{A}_{0}\right|+\left|\partial^{\left(k_{2}-d\right)} \mathcal{B}_{0}\right| \geq\left|\partial^{\left(k_{1}-d\right)} \mathcal{A}^{\prime}\right|+\left|\partial^{\left(k_{2}-d\right)} \mathcal{B}^{\prime}\right|$ for any $d \in\left[1, k_{1}\right]$.

Proof Assume $X=[n]$. Construct set systems $\mathcal{F}$ and $\mathcal{G}$ as follows:

$$
\begin{aligned}
\mathcal{F}= & {\left[n+k_{2}-k_{1}\right]^{\left(k_{2}+1\right)} } \\
& \cup\left(\left[n+k_{2}-k_{1}-1\right]^{\left(k_{2}\right)}+\left\{n+k_{2}-k_{1}+1\right\}\right) \\
& \cup\left(\left[n+k_{2}-k_{1}-2\right]^{\left(k_{2}-1\right)}+\left\{n+k_{2}-k_{1}, n+k_{2}-k_{1}+1\right\}\right) \\
& \cup \cdots \\
& \cup\left([n]^{\left(k_{1}+1\right)}+\left\{n+2, \cdots, n+k_{2}-k_{1}+1\right\}\right) \\
& \cup\left(\mathcal{L}\left([n]^{\left(k_{1}\right)},\left|\mathcal{A}_{0}\right|\right)+\left\{n+1, \cdots, n+k_{2}-k_{1}+1\right\}\right) \\
& \cup\left(\mathcal{L}\left([n]^{\left(k_{2}\right)},\left|\mathcal{B}_{0}\right|\right)+\left\{n+k_{2}-k_{1}+2\right\}\right)
\end{aligned}
$$




$$
\begin{aligned}
\mathcal{G}= & {\left[n+k_{2}-k_{1}\right]^{\left(k_{2}+1\right)} } \\
& \cup\left(\left[n+k_{2}-k_{1}-1\right]^{\left(k_{2}\right)}+\left\{n+k_{2}-k_{1}+1\right\}\right) \\
& \cup\left(\left[n+k_{2}-k_{1}-2\right]^{\left(k_{2}-1\right)}+\left\{n+k_{2}-k_{1}, n+k_{2}-k_{1}+1\right\}\right) \\
& \cup \cdots \\
& \cup\left([n]^{\left(k_{1}+1\right)}+\left\{n+2, \cdots, n+k_{2}-k_{1}+1\right\}\right) \\
& \cup\left(\mathcal{L}\left([n]^{\left(k_{1}\right)},\left|\mathcal{A}^{\prime}\right|\right)+\left\{n+1, \cdots, n+k_{2}-k_{1}+1\right\}\right) \\
& \cup\left(\mathcal{L}\left([n]^{\left(k_{2}\right)},\left|\mathcal{B}^{\prime}\right|\right)+\left\{n+k_{2}-k_{1}+2\right\}\right)
\end{aligned}
$$

Then

$$
\begin{aligned}
& \left|\partial^{\left(k_{2}+1-d\right)} \mathcal{F}\right|=\left(\begin{array}{c}
n+k_{2}-k_{1} \\
k_{2}+1-d
\end{array}\right)+\left(\begin{array}{c}
n+k_{2}-k_{1}-1 \\
k_{2}-d
\end{array}\right)+\left(\begin{array}{c}
n+k_{2}-k_{1}-2 \\
k_{2}-1-d
\end{array}\right)+\cdots+\left(\begin{array}{c}
n \\
k_{1}-d
\end{array}\right) \\
& +\left|\partial^{\left(k_{1}-d\right)} \mathcal{A}_{0}\right|+\left|\partial^{\left(k_{2}-d\right)} \mathcal{B}_{0}\right| \\
& \left|\partial^{\left(k_{2}+1-d\right)} \mathcal{G}\right|=\left(\begin{array}{c}
n+k_{2}-k_{1} \\
k_{2}+1-d
\end{array}\right)+\left(\begin{array}{c}
n+k_{2}-k_{1}-1 \\
k_{2}-d
\end{array}\right)+\left(\begin{array}{c}
n+k_{2}-k_{1}-2 \\
k_{2}-1-d
\end{array}\right)+\cdots+\left(\begin{array}{c}
n \\
k_{1}-d
\end{array}\right) \\
& +\left|\partial^{\left(k_{1}-d\right)} \mathcal{A}^{\prime}\right|+\left|\partial^{\left(k_{2}-d\right)} \mathcal{B}^{\prime}\right|
\end{aligned}
$$

Note that $\mathcal{G}$ is an initial segment in COLEX, so $\left|\partial^{\left(k_{2}+1-d\right)} \mathcal{F}\right| \geq\left|\partial^{\left(k_{2}+1-d\right)} \mathcal{G}\right|$. After cancelling some terms, we obtain the desired inequality.

Theorem 4.1.1 says that for each positive integer $s$, the last $s$ sets in COLEX on $X^{\left(k_{1}\right)}$ add no more to the size of the shadow than any $s$ additional sets in $X^{\left(k_{2}\right)}$ add to the size of the shadow, if $k_{1} \leq k_{2}$.

\subsection{Shadows of two set systems}

Problem. Let $\mathcal{A}, \mathcal{B} \subseteq[n]^{(k)},|\mathcal{A}|+|\mathcal{B}|=r,|\mathcal{A}| \geq|\mathcal{B}| \geq t, 2 t<r, 1 \leq l<k$, find $\min \left|\partial^{(l)} \mathcal{A}\right|+$ $\left|\partial^{(l)} \mathcal{B}\right|$.

Solution. Let $\mathcal{A}^{*}$ and $\mathcal{B}^{*}$ be an optimal configuration. By the Kruskal-Katona theorem, we can assume both $\mathcal{A}^{*}$ and $\mathcal{B}^{*}$ are the initial segments of $[n]^{(k)}$ in COLEX order, so we only need to decide $\left|\mathcal{A}^{*}\right|$ and $\left|\mathcal{B}^{*}\right|$. The following theorem gives one optimal configuration. 
Theorem 4.2.1. Let $\mathcal{A}, \mathcal{B} \subseteq[n]^{(k)},|\mathcal{A}|+|\mathcal{B}|=r,|\mathcal{A}| \geq|\mathcal{B}| \geq t, 2 t<r, 1 \leq l<k$. Let $t=b^{(k)}\left(m_{k}, m_{k-1}, \cdots, m_{h}\right), r-t=b^{(k)}\left(n_{k}, n_{k-1}, \cdots, n_{g}\right)$. Let $p$ be the largest integer such that $n_{p}>m_{p}$ (p exists since $\left.r-t>t\right)$. Let $q$ be the largest integer such that $q<p$ and $R(k, r-t, q+1) \leq\left(\begin{array}{c}m_{q+1} \\ q\end{array}\right)$. Let $\mathcal{A}^{*}$ and $\mathcal{B}^{*}$ be initial segments of $[n]^{(k)}$ in COLEX.

(1) If $R(k, r-t, q+1)+R(k, t, q+1) \leq\left(\begin{array}{c}m_{q+1} \\ q\end{array}\right)$, let $\left|\mathcal{A}^{*}\right|=P(k, r-t, q+1),\left|\mathcal{B}^{*}\right|=r-\left|\mathcal{A}^{*}\right|$.

(2) If $R(k, r-t, q+1)+R(k, t, q+1)>\left(\begin{array}{c}m_{q+1} \\ q\end{array}\right)$, let $\left|\mathcal{B}^{*}\right|=P(k, t, q+1)+\left(\begin{array}{c}m_{q+1} \\ q\end{array}\right),|\mathcal{A}|=r-\left|\mathcal{B}^{*}\right|$.

(3) If $q$ does not exist, let $\left|\mathcal{A}^{*}\right|=r-t,\left|\mathcal{B}^{*}\right|=t$. For convenience, if $q$ does not exist, we say $q=0$

Then $\left|\partial^{(l)} \mathcal{A}\right|+\left|\partial^{(l)} \mathcal{B}\right| \geq\left|\partial^{(l)} \mathcal{A}^{*}\right|+\left|\partial^{(l)} \mathcal{B}^{*}\right|$.

To prove this theorem, we need some lemmas.

Lemma 4.2.2. $\left|\partial^{(l)} \mathcal{L}\left(X^{(k)}, a\right)\right|+\left|\partial^{(l)} \mathcal{L}\left(X^{(k)}, b\right)\right| \geq\left|\partial^{(l)} \mathcal{L}\left(X^{(k)}, a+b\right)\right|$.

Proof Choose a positive integer $n$ with $\left(\begin{array}{l}n \\ k\end{array}\right) \geq a+b$. Let

$$
\begin{aligned}
& \mathcal{F}=\mathcal{L}\left(X^{(k+1)},\left(\begin{array}{c}
n \\
k+1
\end{array}\right)\right) \cup\left[\mathcal{L}\left(X^{(k)}, a\right)+\{n+1\}\right] \cup\left[\mathcal{L}\left(X^{(k)}, b\right)+\{n+2\}\right], \\
& \mathcal{G}=\mathcal{L}\left(X^{(k+1)},\left(\begin{array}{c}
n \\
k+1
\end{array}\right)\right) \cup\left[\mathcal{L}\left(X^{(k)}, a+b\right)+\{n+1\}\right] .
\end{aligned}
$$

Then $\left|\partial^{(l+1)} \mathcal{F}\right|=\left(\begin{array}{c}n \\ l+1\end{array}\right)+\left|\partial^{(l)} \mathcal{L}\left(X^{(k)}, a\right)\right|+\left|\partial^{(l)} \mathcal{L}\left(X^{(k)}, b\right)\right|$,

$$
\left|\partial^{(l+1)} \mathcal{G}\right|=\left(\begin{array}{c}
n \\
l+1
\end{array}\right)+\left|\partial^{(l)} \mathcal{L}\left(X^{(k)}, a+b\right)\right| .
$$

Because $\mathcal{G}$ is the initial segment of the COLEX order, by the Kruskal-Katona Theorem, $\left|\partial^{(l+1)} \mathcal{F}\right| \geq\left|\partial^{(l+1)} \mathcal{G}\right|$. That is,

$$
\left(\begin{array}{c}
n \\
l+1
\end{array}\right)+\left|\partial^{(l)} \mathcal{L}\left(X^{(k)}, a\right)\right|+\left|\partial^{(l)} \mathcal{L}\left(X^{(k)}, b\right)\right| \geq\left(\begin{array}{c}
n \\
l+1
\end{array}\right)+\left|\partial^{(l)} \mathcal{L}\left(X^{(k)}, a+b\right)\right| .
$$

After cancelling $\left(\begin{array}{c}n \\ l+1\end{array}\right)$, we obtain the desired inequality.

Lemma 4.2.3. If $a<\left(\begin{array}{c}w \\ k\end{array}\right), b<\left(\begin{array}{c}w \\ k\end{array}\right)$, and $a+b>\left(\begin{array}{c}w \\ k\end{array}\right)$, then $\left|\partial^{(l)} \mathcal{L}\left(X^{(k)}, a\right)\right|+\left|\partial^{(l)} \mathcal{L}\left(X^{(k)}, b\right)\right| \geq$ $\left|\partial^{(l)} \mathcal{L}\left(X^{(k)},\left(\begin{array}{c}w \\ k\end{array}\right)\right)\right|+\left|\partial^{(l)} \mathcal{L}\left(X^{(k)}, a+b-\left(\begin{array}{c}w \\ k\end{array}\right)\right)\right|$. 


\section{Proof Let}

$$
\begin{aligned}
& \mathcal{F}=\mathcal{L}\left(X^{(k+1)},\left(\begin{array}{c}
w \\
k+1
\end{array}\right)\right) \cup\left[\mathcal{L}\left(X^{(k)}, a\right)+\{w+1\}\right] \cup\left[\mathcal{L}\left(X^{(k)}, b\right)+\{w+2\}\right] \\
& \mathcal{G}=\mathcal{L}\left(X^{(k+1)},\left(\begin{array}{c}
w \\
k+1
\end{array}\right)\right) \cup\left[\mathcal{L}\left(X^{(k)},\left(\begin{array}{c}
w \\
k
\end{array}\right)\right)+\{w+1\}\right] \cup\left[\mathcal{L}\left(X^{(k)}, a+b-\left(\begin{array}{c}
w \\
k
\end{array}\right)\right)+\{w+2\}\right] .
\end{aligned}
$$

By the same argument in Lemma 4.2.2, we obtain the desired inequality.

Lemma 4.2.4. If $a \geq\left(\begin{array}{l}w \\ k\end{array}\right),\left(\begin{array}{c}w \\ k\end{array}\right)<b \leq\left(\begin{array}{c}w+1 \\ k\end{array}\right)$, then

$$
\left|\partial^{(l)} \mathcal{L}\left(X^{(k)}, a\right)\right|+\left|\partial^{(l)} \mathcal{L}\left(X^{(k)}, b\right)\right| \geq\left|\partial^{(l)} \mathcal{L}\left(X^{(k)}, a+b-\left(\begin{array}{l}
w \\
k
\end{array}\right)\right)\right|+\left|\partial^{(l)} \mathcal{L}\left(X^{(k)},\left(\begin{array}{l}
w \\
k
\end{array}\right)\right)\right|
$$

Proof Let $b=\left(\begin{array}{c}w \\ k\end{array}\right)+s$, then $\left|\partial^{(l)} \mathcal{L}\left(X^{(k)}, b\right)\right|=\left|\partial^{(l)} \mathcal{L}\left(X^{(k)},\left(\begin{array}{c}w \\ k\end{array}\right)\right)\right|+\left|\partial^{(l-1)} \mathcal{L}\left(X^{(k-1)}, s\right)\right|$. So the inequality is equivalent to

$$
\left|\partial^{(l)} \mathcal{L}\left(X^{(k)}, a\right)\right|+\left|\partial^{(l-1)} \mathcal{L}\left(X^{(k-1)}, s\right)\right| \geq\left|\partial^{(l)} \mathcal{L}\left(X^{(k)}, a+s\right)\right| .
$$

Let $\mathcal{F}=\mathcal{L}\left(X^{(k)}, a\right) \cup\left[\mathcal{L}\left(X^{(k-1)}, s\right)+\{x\}\right]$ where $x$ is a new symbol. Then $\left|\partial^{(l)} \mathcal{F}\right|=$ $\left|\partial^{(l)} \mathcal{L}\left(X^{(k)}, a\right)\right|+\left|\partial^{(l-1)} \mathcal{L}\left(X^{(k-1)}, s\right)\right|$. By the Kruskal-Katona Theorem, we obtain the above inequality.

Now we are ready to prove Theorem 4.2.1.

Proof of Theorem 4.2.1 By way of contradiction, assume $\mathcal{A}^{*}$ and $\mathcal{B}^{*}$ are not optimal. Choose an optimal pair $(\mathcal{A}, \mathcal{B})$ such that || $\mathcal{B}|-| \mathcal{B}^{*}||$ is minimum. Again, we can assume both $\mathcal{A}$ and $\mathcal{B}$ are the initial segments of $[n]^{(k)}$ in COLEX order.

Let $u$ be the largest integer such that $|\mathcal{B}|>C(k, t, u)$.

(1) If $u>p$

Then $|\mathcal{B}|>P(k, t, u)+\left(\begin{array}{c}m_{u} \\ u-1\end{array}\right)$, so $|\mathcal{A}|+|\mathcal{B}|>2|\mathcal{B}|>2\left[P(k, t, u)+\left(\begin{array}{c}m_{u} \\ u-1\end{array}\right)\right]$. But then 


$$
\begin{aligned}
r & =t+(r-t) \\
& <P(k, t, u)+\left(\begin{array}{c}
m_{u} \\
u-1
\end{array}\right)+P(k, r-t, u)+\left(\begin{array}{c}
n_{u} \\
u-1
\end{array}\right) \\
& =P(k, t, u)+\left(\begin{array}{c}
m_{u} \\
u-1
\end{array}\right)+P(k, t, u)+\left(\begin{array}{c}
m_{u} \\
u-1
\end{array}\right) \\
& =2\left[P(k, t, u)+\left(\begin{array}{c}
m_{u} \\
u-1
\end{array}\right)\right]
\end{aligned}
$$

It's a contradiction to $|\mathcal{A}|+|\mathcal{B}|=r$.

(2) If $u=p$

By definition of $p$, we have $P(k,|\mathcal{B}|, u+1)=P(k,|\mathcal{A}|, u+1)$. So $R(k,|\mathcal{B}|, u+1) \leq$ $R(k,|\mathcal{A}|, u+1)$.

Let $w$ be the largest integer such that $R(k,|\mathcal{B}|, u+1)>\left(\begin{array}{l}w \\ u\end{array}\right)$. So $R(k,|\mathcal{B}|, u+1) \leq\left(\begin{array}{c}w+1 \\ u\end{array}\right)$ and $R(k,|\mathcal{A}|, u+1) \geq\left(\begin{array}{l}w \\ u\end{array}\right)$.

Let $\mathcal{A}^{\prime}=\mathcal{L}\left(X^{(k)},|\mathcal{A}|+R(k,|\mathcal{B}|, u+1)-\left(\begin{array}{l}w \\ u\end{array}\right)\right)$

$$
\mathcal{B}^{\prime}=\mathcal{L}\left(X^{(k)}, P(k,|\mathcal{B}|, u+1)+\left(\begin{array}{l}
w \\
u
\end{array}\right)\right) .
$$

Because $\mathcal{L}\left(X^{(k)}, P(k, r-t, u+1)\right)$ is contained in both $\mathcal{A}$ and $\mathcal{A}^{\prime}$, and $\mathcal{L}\left(X^{(k)}, P(k, t, u+1)\right)$ is contained in both $\mathcal{B}$ and $\mathcal{B}^{\prime}$, we know that $\left|\partial^{(l)} \mathcal{A}\right|+\left|\partial^{(l)} \mathcal{B}\right| \geq\left|\partial^{(l)} \mathcal{A}^{\prime}\right|+\left|\partial^{(l)} \mathcal{B}^{\prime}\right|$ is equivalent to $\left|\partial^{(u-k+l)} \mathcal{L}\left(X^{(u)}, R(k,|\mathcal{A}|, u+1)\right)\right|+\left|\partial^{(u-k+l)} \mathcal{L}\left(X^{(u)}, R(k,|\mathcal{B}|, u+1)\right)\right| \geq\left|\partial^{(u-k+l)} \mathcal{L}\left(X^{(u)},\left(\begin{array}{l}w \\ u\end{array}\right)\right)\right|+$ $\left|\partial^{(u-k+l)} \mathcal{L}\left(X^{(u)}, R(k,|\mathcal{A}|, u+1)+R(k,|\mathcal{B}|, u+1)-\left(\begin{array}{l}w \\ u\end{array}\right)\right)\right|$. This follows from Lemma 4.2.4.

So $\left(\mathcal{A}^{\prime}, \mathcal{B}^{\prime}\right)$ is a contradiction to the choice of $\mathcal{A}$ and $\mathcal{B}$.

(3) If $q<u<p$

If $R(k,|\mathcal{B}|, u+1) \leq R(k,|\mathcal{A}|, u+1)$, By the same argument in (2), we again obtain a contradiction to the choice of $\mathcal{A}$ and $\mathcal{B}$. So assume $R(k,|\mathcal{B}|, u+1)>R(k,|\mathcal{A}|, u+1)$. Let 
$\mathcal{A}^{\prime}=\mathcal{L}\left(X^{(k)}, P(k,|\mathcal{A}|, u+1)+R(k,|\mathcal{B}|, u+1)\right), \mathcal{B}^{\prime}=\mathcal{L}\left(X^{(k)}, P(k,|\mathcal{B}|, u+1)+R(k,|\mathcal{A}|, u+1)\right)$. It is easy to verify that $\left|\partial^{(l)} \mathcal{A}\right|+\left|\partial^{(l)} \mathcal{B}\right|=\left|\partial^{(l)} \mathcal{A}^{\prime}\right|+\left|\partial^{(l)} \mathcal{B}^{\prime}\right|$ and $\left|\mathcal{B}^{\prime}\right|<|\mathcal{B}|$.

By definition of $q, R(k, r-t, u+1)>\left(\begin{array}{c}m_{u+1} \\ u\end{array}\right)$. By definition of $u$, we know $P(k, t, u+1)=$ $P(k,|\mathcal{B}|, u+1)$ and $|\mathcal{B}| \leq P(k, t, u+1)+\left(\begin{array}{c}m_{u+1} \\ u\end{array}\right)$. Therefore $R(k,|\mathcal{A}|, u+1)>t-P(k,|\mathcal{B}|, u+1)$. It follows that $\left|\mathcal{B}^{\prime}\right|>t$. So $\left(\mathcal{A}^{\prime}, \mathcal{B}^{\prime}\right)$ is a valid pair, contradicting the choice of $\mathcal{A}$ and $\mathcal{B}$.

(4) If $u \leq q$

Then $|\mathcal{B}| \leq P(k, t, q+1)+\left(\begin{array}{c}m_{q+1} \\ q\end{array}\right)$.

a. If $R(k, r-t, q+1)+R(k, t, q+1)>\left(\begin{array}{c}m_{q+1} \\ q\end{array}\right)$.

Because $\mathcal{L}\left(X^{(k)}, P(k, r-t, q+1)\right)$ is contained in both $\mathcal{A}$ and $\mathcal{A}^{*}$, and $\mathcal{L}\left(X^{(k)}, P(k, t, q+1)\right)$ is contained in both $\mathcal{B}$ and $\mathcal{B}^{*}$, we know that $\left|\partial^{(l)} \mathcal{A}\right|+\left|\partial^{(l)} \mathcal{B}\right| \geq\left|\partial^{(l)} \mathcal{A}^{*}\right|+\left|\partial^{(l)} \mathcal{B}^{*}\right|$ is equivalent to $\left|\partial^{(q-k+l)} \mathcal{L}\left(X^{(q)}, R(k,|\mathcal{A}|, q+1)\right)\right|+\left|\partial^{(q-k+l)} \mathcal{L}\left(X^{(q)}, R(k,|\mathcal{B}|, q+1)\right)\right| \geq\left|\partial^{(q-k+l)} \mathcal{L}\left(X^{(q)},\left(\begin{array}{c}m_{q+1} \\ q\end{array}\right)\right)\right|+$

$\left|\partial^{(q-k+l)} \mathcal{L}\left(X^{(q)}, R(k,|\mathcal{A}|, q+1)+R(k,|\mathcal{B}|, q+1)-\left(\begin{array}{c}m_{q+1} \\ q\end{array}\right)\right)\right|$. This follows from Lemma 4.2.3.

b. If $R(k, r-t, q+1)+R(k, t, q+1) \leq\left(\begin{array}{c}m_{q+1} \\ q\end{array}\right)$.

b1. $|\mathcal{A}| \geq P(k, r-t, q+1)$

By the same argument in a, it suffices to show that $\left|\partial^{(q-k+l)} \mathcal{L}\left(X^{(q)}, R(k,|\mathcal{A}|, q+1)\right)\right|+$ $\left|\partial^{(q-k+l)} \mathcal{L}\left(X^{(q)}, R(k,|\mathcal{B}|, q+1)\right)\right| \geq\left|\partial^{(q-k+l)} \mathcal{L}\left(X^{(q)}, R(k,|\mathcal{A}|, q+1)+R(k,|\mathcal{B}|, q+1)\right)\right|$. This follows from Lemma 4.2.2.

b2. $|\mathcal{A}|<P(k, r-t, q+1)$

By the same argument in (3), we have $R(k, r-t, q+2)>R(k, t, q+2)$. So $R(k, r-t, q+2)>$ $\left(\begin{array}{c}m_{q+1} \\ q+1\end{array}\right)$. Now by the same argument in a, it suffices to show that

$$
\begin{aligned}
& \quad\left|\partial^{(q-k+l)} \mathcal{L}\left(X^{(q)}, R(k,|\mathcal{A}|, q+1)\right)\right|+\left|\partial^{(q-k+l)} \mathcal{L}\left(X^{(q)}, R(k,|\mathcal{B}|, q+1)\right)\right| \\
& \geq\left|\partial^{(q-k+l)} \mathcal{L}\left(X^{(q)},\left(\begin{array}{c}
n_{q+1}-1 \\
q
\end{array}\right)\right)\right|+\mid \partial^{(q-k+l)} \mathcal{L}\left(X^{(q)}, R(k,|\mathcal{A}|, q+1)\right. \\
& \left.\quad+R(k,|\mathcal{B}|, q+1)-\left(\begin{array}{c}
n_{q+1}-1 \\
q
\end{array}\right)\right) \mid
\end{aligned}
$$


It follows again from Lemma 4.2.3.

\subsection{Minimum shadow with degree conditions}

In this section, we will prove some results on minimum shadow problems with the restriction that the degrees of the vertices cannot be too big. For simplicity, we state the theorems in terms of the first order shadow, but they are true for $l$-shadow for general $l$.

Lemma 4.3.1. Let $X=[n+2], \mathcal{F} \subseteq X^{(k)}$ be a left compressed set system. $|\mathcal{F}|=\left(\begin{array}{l}n \\ k\end{array}\right)+\left(\begin{array}{c}n-1 \\ k-1\end{array}\right)+t$, $0<t \leq\left(\begin{array}{c}n-1 \\ k-1\end{array}\right)$. If $\forall i \in X, c(i) \leq\left(\begin{array}{l}n \\ k\end{array}\right)+\left(\begin{array}{c}n-1 \\ k-1\end{array}\right)$, then $|\partial \mathcal{F}| \geq\left|\partial \mathcal{F}^{*}\right|$, where $\mathcal{F}^{*}=\mathcal{L}\left(X^{(k)},\left(\begin{array}{l}n \\ k\end{array}\right)+\right.$ $\left.\left(\begin{array}{l}n-1 \\ k-1\end{array}\right)\right) \cup\left[\mathcal{L}\left(X^{(k-1)}, t\right)+\{n+2\}\right]$.

Proof Use induction on $k$ and $|\mathcal{F}|$.

When $k=2,|\partial \mathcal{F}|=$ number of symbols used in $\mathcal{F}$. By the degree condition, we have $|\partial \mathcal{F}|=\left|\partial \mathcal{F}^{*}\right|$

Assume the theorem is true for smaller $k$ and $|\mathcal{F}|$. Let $t=b^{(k-1)}\left(m_{k-1}, \cdots, m_{s}\right)$, then

$$
\begin{aligned}
\left|\partial \mathcal{F}^{*}\right|= & \left(\begin{array}{c}
n \\
k-1
\end{array}\right)+\left(\begin{array}{c}
n-1 \\
k-2
\end{array}\right)+b^{(k-2)}\left(m_{k-1}, \cdots, m_{s}\right) . \\
|\mathcal{F}|= & \left(\begin{array}{c}
n-1 \\
k-1
\end{array}\right)+\left(\begin{array}{c}
n-2 \\
k-2
\end{array}\right)+b^{(k-2)}\left(m_{k-1}-1, \cdots, m_{s}-1\right)+\left(\begin{array}{c}
n-1 \\
k
\end{array}\right)+\left(\begin{array}{c}
n-2 \\
k-1
\end{array}\right) \\
& +b^{(k-1)}\left(m_{k-1}-1, \cdots, m_{s}-1\right)
\end{aligned}
$$

Since $\mathcal{F}$ is left compressed, we have $\partial \mathcal{F}_{\overline{1}} \subseteq \mathcal{F}(1)$.

Case 1. $\left|\mathcal{F}_{\overline{1}}\right|>\left(\begin{array}{c}n-1 \\ k\end{array}\right)+\left(\begin{array}{c}n-2 \\ k-1\end{array}\right)+b^{(k-1)}\left(m_{k-1}-1, \cdots, m_{s}-1\right)$

1(a). $\exists i \geq 2$ such that $c_{\mathcal{F}_{\overline{1}}}(i)>\left(\begin{array}{c}n-1 \\ k\end{array}\right)+\left(\begin{array}{c}n-2 \\ k-1\end{array}\right)$. 
By Kruskal-Katona Theorem, $\left|\partial \mathcal{F}_{\overline{1}, \bar{i}}\right| \geq\left(\begin{array}{c}n-1 \\ k-1\end{array}\right)+\left(\begin{array}{c}n-2 \\ k-2\end{array}\right)$, thus

$$
\left|\mathcal{F}_{1, \bar{i}}\right| \geq\left|\partial \mathcal{F}_{\overline{1}, \bar{i}}\right| \geq\left(\begin{array}{l}
n-1 \\
k-1
\end{array}\right)+\left(\begin{array}{l}
n-2 \\
k-2
\end{array}\right)
$$

Therefore $\left|\partial \mathcal{F}_{1, \bar{i}}\right| \geq\left(\begin{array}{c}n-1 \\ k-1\end{array}\right)+\left(\begin{array}{c}n-2 \\ k-3\end{array}\right)$ by Kruskal-Katona Theorem. Now

$|\partial(\mathcal{F}, \bar{i})|=\left|\partial \mathcal{F}_{\overline{1}, \bar{i}}\right|+\left|\partial \mathcal{F}_{1, \bar{i}}\right| \geq\left(\begin{array}{c}n-1 \\ k-1\end{array}\right)+\left(\begin{array}{c}n-2 \\ k-2\end{array}\right)+\left(\begin{array}{c}n-1 \\ k-2\end{array}\right)+\left(\begin{array}{c}n-2 \\ k-3\end{array}\right)=\left(\begin{array}{c}n \\ k-1\end{array}\right)+\left(\begin{array}{c}n-1 \\ k-2\end{array}\right)$.

On the other hand, the degree condition yields $\left|\mathcal{F}_{i}\right| \geq t$, hence

$$
|\partial(\mathcal{F}, i)| \geq b^{(k-2)}\left(m_{k-1}, \cdots, m_{s}\right)
$$

Therefore

$$
|\partial \mathcal{F}|=|\partial(\mathcal{F}, \bar{i})|+|\partial(\mathcal{F}, i)| \geq\left(\begin{array}{c}
n \\
k-1
\end{array}\right)+\left(\begin{array}{l}
n-1 \\
k-2
\end{array}\right)+b^{(k-2)}\left(m_{k-1}, \cdots, m_{s}\right)=\left|\partial \mathcal{F}^{*}\right|
$$

$1(\mathrm{~b}) . c_{\mathcal{F}_{\overline{1}}}(i) \leq\left(\begin{array}{c}n-1 \\ k\end{array}\right)+\left(\begin{array}{c}n-2 \\ k-1\end{array}\right) \forall i \geq 2$.

By inductive hypothesis, $\left|\partial \mathcal{F}_{\overline{1}}\right| \geq\left(\begin{array}{c}n-1 \\ k-1\end{array}\right)+\left(\begin{array}{c}n-2 \\ k-2\end{array}\right)+b^{(k-2)}\left(m_{k-1}-1, \cdots, m_{s}-1\right)$.

So

$$
\begin{aligned}
|\mathcal{F}|= & \left|\mathcal{F}_{1}\right|+\left|\mathcal{F}_{\overline{1}}\right| \\
\geq & \left|\partial \mathcal{F}_{\overline{1}}\right|+\left|\mathcal{F}_{\overline{1}}\right| \\
> & \left(\begin{array}{c}
n-1 \\
k-1
\end{array}\right)+\left(\begin{array}{l}
n-2 \\
k-2
\end{array}\right)+b^{(k-2)}\left(m_{k-1}-1, \cdots, m_{s}-1\right) \\
& +\left(\begin{array}{c}
n-1 \\
k
\end{array}\right)+\left(\begin{array}{c}
n-2 \\
k-1
\end{array}\right)+b^{(k-1)}\left(m_{k-1}-1, \cdots, m_{s}-1\right) \\
= & \left(\begin{array}{c}
n \\
k
\end{array}\right)+\left(\begin{array}{c}
n-1 \\
k-1
\end{array}\right)+b^{(k-1)}\left(m_{k-1}, \cdots, m_{s}\right)
\end{aligned}
$$


It's a contradiction to the assumption on $|\mathcal{F}|$.

Case 2. $\left|\mathcal{F}_{\overline{1}}\right| \leq\left(\begin{array}{c}n-1 \\ k\end{array}\right)+\left(\begin{array}{c}n-2 \\ k-1\end{array}\right)+b^{(k-1)}\left(m_{k-1}-1, \cdots, m_{s}-1\right)$

Then $\left|\mathcal{F}_{1}\right| \geq\left(\begin{array}{l}n-1 \\ k-1\end{array}\right)+\left(\begin{array}{c}n-2 \\ k-2\end{array}\right)+b^{(k-2)}\left(m_{k-1}-1, \cdots, m_{s}-1\right)$

2(a). $\exists i \geq 2$ such that $c_{\mathcal{F}_{1}}(i)>\left(\begin{array}{c}n-1 \\ k-1\end{array}\right)+\left(\begin{array}{c}n-2 \\ k-2\end{array}\right)$.

By a similar argument to $1\left(\right.$ a), we can show $|\partial \mathcal{F}| \geq\left|\partial \mathcal{F}^{*}\right|$.

$2(\mathrm{~b}) . c_{\mathcal{F}_{1}}(i) \leq\left(\begin{array}{l}n-1 \\ k-1\end{array}\right)+\left(\begin{array}{c}n-2 \\ k-2\end{array}\right) \forall i \geq 2$.

By inductive hypothesis, $|\partial \mathcal{F}(1)| \geq\left(\begin{array}{l}n-1 \\ k-2\end{array}\right)+\left(\begin{array}{l}n-2 \\ k-3\end{array}\right)+b^{(k-3)}\left(m_{k-1}-1, \cdots, m_{s}-1\right)$.

So

$$
\begin{aligned}
\left|\partial \mathcal{F}_{1}\right| \geq & \left|\mathcal{F}_{1}\right|+|\partial \mathcal{F}(1)| \\
\geq & \left(\begin{array}{l}
n-1 \\
k-1
\end{array}\right)+\left(\begin{array}{l}
n-2 \\
k-2
\end{array}\right)+b^{(k-2)}\left(m_{k-1}-1, \cdots, m_{s}-1\right) \\
& +\left(\begin{array}{l}
n-1 \\
k-2
\end{array}\right)+\left(\begin{array}{l}
n-2 \\
k-3
\end{array}\right)+b^{(k-3)}\left(m_{k-1}-1, \cdots, m_{s}-1\right) \\
= & \left(\begin{array}{c}
n \\
k-1
\end{array}\right)+\left(\begin{array}{l}
n-1 \\
k-2
\end{array}\right)+b^{(k-2)}\left(m_{k-1}, \cdots, m_{s}\right)=\left|\partial \mathcal{F}^{*}\right|
\end{aligned}
$$

Definition 4.3.2. Suppose $t=\left(\begin{array}{c}m_{k-1} \\ k-1\end{array}\right)+\left(\begin{array}{c}m_{k-2} \\ k-2\end{array}\right)+\cdots+\left(\begin{array}{c}m_{s+1} \\ s+1\end{array}\right)+\left(\begin{array}{c}m_{s} \\ s\end{array}\right)+\cdots+\left(\begin{array}{c}m_{r} \\ r\end{array}\right)$ is the cascade form of $t$, where $m_{i}>i$ for $s+1 \leq i \leq k-1$ and $m_{i}=i$ for $r \leq i \leq s$. Define $\alpha(t)=\left(\begin{array}{c}m_{k-1}-1 \\ k-1\end{array}\right)+\left(\begin{array}{c}m_{k-2}-1 \\ k-2\end{array}\right)+\cdots+\left(\begin{array}{c}m_{s+1}-1 \\ s+1\end{array}\right)+\left(\begin{array}{c}m_{s} \\ s\end{array}\right), \beta(t)=\left(\begin{array}{c}m_{k-1}-1 \\ k-2\end{array}\right)+\left(\begin{array}{c}m_{k-2}-1 \\ k-3\end{array}\right)+\cdots+$ $\left(\begin{array}{c}m_{s+1}-1 \\ s\end{array}\right)+\left(\begin{array}{c}m_{s-1} \\ s-1\end{array}\right)+\left(\begin{array}{c}m_{s-2} \\ s-2\end{array}\right)+\cdots+\left(\begin{array}{c}m_{r} \\ r\end{array}\right), \gamma(t)=\left(\begin{array}{c}m_{k-1}-2 \\ k-2\end{array}\right)+\left(\begin{array}{c}m_{k-2}-2 \\ k-3\end{array}\right)+\cdots+\left(\begin{array}{c}m_{s+1}-2 \\ s\end{array}\right)+\left(\begin{array}{c}m_{s}-1 \\ s-1\end{array}\right)$. The terms that involve $m_{i}$ for $i \leq s$ will be dropped if they do not exist in the cascade form of $t$.

Clearly, $\alpha(t)+\beta(t)=t$. 
Lemma 4.3.3. Let $X=[n+2], \mathcal{F} \subseteq X^{(k)}$ with $|\mathcal{F}|=\left(\begin{array}{l}n \\ k\end{array}\right)+\left(\begin{array}{l}n-1 \\ k-1\end{array}\right)+t$ where $0<t \leq\left(\begin{array}{l}n-1 \\ k-1\end{array}\right)$. If $\exists i$ with $\alpha(t) \leq d(i) \leq\left(\begin{array}{c}n-1 \\ k-1\end{array}\right)+\beta(t)$, then $|\partial \mathcal{F}| \geq\left|\partial \mathcal{F}^{*}\right|$ where $\mathcal{F}^{*}=\mathcal{L}\left(X^{(k)},\left(\begin{array}{c}n \\ k\end{array}\right)+\left(\begin{array}{c}n-1 \\ k-1\end{array}\right)\right) \cup$ $\left[\mathcal{L}\left(X^{(k-1)}, t\right)+\{n+2\}\right]$.

Proof Without loss of generality, assume $i=n+2$. By Kruskal-Katona Theorem, we may further assume $\mathcal{F}=\mathcal{L}\left(X^{(k)},|\mathcal{F}|-d(n+2)\right) \cup\left[\mathcal{L}\left(X^{(k-1)}, d(n+2)\right)+\{n+2\}\right]$.

Note that $\left|\partial \mathcal{F}^{*}\right|=\left(\begin{array}{c}n \\ k-1\end{array}\right)+\left(\begin{array}{c}n-1 \\ k-2\end{array}\right)+\left|\partial \mathcal{L}\left(X^{(k-1)}, t\right)\right|$.

Case 1. $\alpha(t) \leq d(n+2) \leq t$.

Then $c(n+2) \geq\left(\begin{array}{l}n \\ k\end{array}\right)+\left(\begin{array}{l}n-1 \\ k-1\end{array}\right)$. Let $a=c(n+2)-\left(\begin{array}{l}n \\ k\end{array}\right)-\left(\begin{array}{l}n-1 \\ k-1\end{array}\right)$. So $a+d(n+2)=t$, and

$$
\begin{aligned}
|\partial \mathcal{F}| & =|\partial(\mathcal{F}, \bar{n}+2)|+|\partial(\mathcal{F}, n+2)| \\
& =\left(\begin{array}{c}
n \\
k-1
\end{array}\right)+\left(\begin{array}{l}
n-1 \\
k-2
\end{array}\right)+\left|\partial \mathcal{L}\left(X^{(k-1)}, a\right)\right|+\left|\partial \mathcal{L}\left(X^{(k-1)}, d(n+2)\right)\right|
\end{aligned}
$$

Therefore $|\partial \mathcal{F}| \geq\left|\partial \mathcal{F}^{*}\right|$ is equivalent to $\left|\partial \mathcal{L}\left(X^{(k-1)}, a\right)\right|+\left|\partial \mathcal{L}\left(X^{(k-1)}, d(n+2)\right)\right| \geq\left|\partial \mathcal{L}\left(X^{(k-1)}, t\right)\right|$. Thus the problem reduces to the one in section 4.2. That is, find $\min |\partial \mathcal{A}|+|\partial \mathcal{B}|$ where $|\mathcal{A}|+|\mathcal{B}|=t$ and $|\mathcal{A}| \geq|\mathcal{B}| \geq \alpha(t)$.

Let $p, q$ be defined as in section 4.2. Clearly $n-1>m_{k-1}-1$, so $p=k-1$.

(1) $q \geq s+1$.

$R(k-1, a, q+1)=\left(\begin{array}{c}m_{q+1}-1 \\ q\end{array}\right)+\left(\begin{array}{c}m_{q}-1 \\ q-1\end{array}\right)+\cdots+\left(\begin{array}{c}m_{s+1}-1 \\ s\end{array}\right)+\left(\begin{array}{c}m_{s-1} \\ s-1\end{array}\right)+\left(\begin{array}{c}m_{s-2} \\ s-2\end{array}\right)+\cdots+\left(\begin{array}{c}m_{r} \\ r\end{array}\right)$.

By definition of $q, R(k-1, a, q+1) \leq\left(\begin{array}{c}m_{q+1}-1 \\ q\end{array}\right)$. So $R(k-1, a, q+1)=\left(\begin{array}{c}m_{q+1}-1 \\ q\end{array}\right)$

It follows that $\beta(t)=\left(\begin{array}{c}m_{k-1}-1 \\ k-2\end{array}\right)+\left(\begin{array}{c}m_{k-2}-1 \\ k-3\end{array}\right)+\cdots+\left(\begin{array}{c}m_{q+1}-1 \\ q\end{array}\right)$,

$$
\alpha(t)=\left(\begin{array}{c}
m_{k-1}-1 \\
k-1
\end{array}\right)+\left(\begin{array}{c}
m_{k-2}-1 \\
k-2
\end{array}\right)+\cdots+\left(\begin{array}{c}
m_{q+1}-1 \\
q+1
\end{array}\right) .
$$


By the theorem in section 4.2, the optimal solution is

$$
\begin{gathered}
\left|\mathcal{A}^{*}\right|=\left(\begin{array}{c}
m_{k-1}-1 \\
k-2
\end{array}\right)+\left(\begin{array}{c}
m_{k-2}-1 \\
k-3
\end{array}\right)+\cdots+\left(\begin{array}{c}
m_{q+2}-1 \\
q+1
\end{array}\right), \\
\left|\mathcal{B}^{*}\right|=\left(\begin{array}{c}
m_{k-1}-1 \\
k-1
\end{array}\right)+\left(\begin{array}{c}
m_{k-2}-1 \\
k-2
\end{array}\right)+\cdots+\left(\begin{array}{c}
m_{q+1}-1 \\
q+1
\end{array}\right)+\left(\begin{array}{c}
m_{q+1}-1 \\
q
\end{array}\right) .
\end{gathered}
$$

It can be easily verified that $\left|\partial \mathcal{A}^{*}\right|+\left|\partial \mathcal{B}^{*}\right|=\left|\partial \mathcal{L}\left(X^{(k-1)}, t\right)\right|$.

This completes the proof of $\left|\partial \mathcal{L}\left(X^{(k-1)}, a\right)\right|+\left|\partial \mathcal{L}\left(X^{(k-1)}, d(n+2)\right)\right| \geq\left|\partial \mathcal{L}\left(X^{(k-1)}, t\right)\right|$.

(2) $0<q \leq s$

By a similar argument to (1), we can show

$$
\left|\partial \mathcal{L}\left(X^{(k-1)}, a\right)\right|+\left|\partial \mathcal{L}\left(X^{(k-1)}, d(n+2)\right)\right| \geq\left|\partial \mathcal{L}\left(X^{(k-1)}, t\right)\right| .
$$

(3) $q=0$.

By the theorem in section 4.2 , the optimal solution is $\left|\mathcal{A}^{*}\right|=\beta(t),\left|\mathcal{B}^{*}\right|=\alpha(t)$, and again it can be easily verified that $\left|\partial \mathcal{A}^{*}\right|+\left|\partial \mathcal{B}^{*}\right|=\left|\partial \mathcal{L}\left(X^{(k-1)}, t\right)\right|$.

Case 2. $t<d(n+2) \leq\left(\begin{array}{l}n-1 \\ k-1\end{array}\right)$.

Then $\left(\begin{array}{l}n \\ k\end{array}\right) \leq c(n+2)<\left(\begin{array}{l}n \\ k\end{array}\right)+\left(\begin{array}{l}n-1 \\ k-1\end{array}\right)$. Let $a=c(n+2)-\left(\begin{array}{l}n \\ k\end{array}\right)$. Then $a<\left(\begin{array}{l}n-1 \\ k-1\end{array}\right)$ and $a+d(n+2)=\left(\begin{array}{c}n-1 \\ k-1\end{array}\right)+t$.

By Lemma 4.2.3 in section 4.2,

$$
\left|\partial \mathcal{L}\left(X^{(k-1)}, a\right)\right|+\left|\partial \mathcal{L}\left(X^{(k-1)}, d(n+2)\right)\right| \geq\left(\begin{array}{c}
n-1 \\
k-2
\end{array}\right)+\left|\partial \mathcal{L}\left(X^{(k-1)}, t\right)\right|
$$


Therefore

$$
\begin{aligned}
|\partial \mathcal{F}| & =|\partial(\mathcal{F}, \overline{n+2})|+|\partial(\mathcal{F}, n+2)| \\
& =\left(\begin{array}{c}
n \\
k-1
\end{array}\right)+\left|\partial \mathcal{L}\left(X^{(k-1)}, a\right)\right|+\left|\partial \mathcal{L}\left(X^{(k-1)}, d(n+2)\right)\right| \\
& \geq\left(\begin{array}{c}
n \\
k-1
\end{array}\right)+\left(\begin{array}{c}
n-1 \\
k-2
\end{array}\right)+\left|\partial \mathcal{L}\left(X^{(k-1)}, t\right)\right| \\
& =\left|\partial \mathcal{F}^{*}\right|
\end{aligned}
$$

Case 3. $\left(\begin{array}{l}n-1 \\ k-1\end{array}\right)<d(n+2) \leq\left(\begin{array}{c}n-1 \\ k-1\end{array}\right)+\beta(t)$.

Then $c(n+2) \geq \alpha(t)$. Let $b=d(n+2)-\left(\begin{array}{c}n-1 \\ k-1\end{array}\right)$. Then $b+c(n+2)=t$. It is the same situation as Case 1 .

Lemma 4.3.4. Let $X=[n+2], \mathcal{F} \subseteq X^{(k)}$ with $|\mathcal{F}|=\left(\begin{array}{l}n \\ k\end{array}\right)+\left(\begin{array}{c}n-1 \\ k-1\end{array}\right)+t$ where $0<t \leq\left(\begin{array}{l}n-1 \\ k-1\end{array}\right)$. If $\exists i$ with $d(i)<\alpha(t)$ and $\exists j$ with $\left|\mathcal{F}_{i} \cap \mathcal{F}_{j}\right| \geq \gamma(t)$, then $|\partial \mathcal{F}| \geq\left|\partial \mathcal{F}^{*}\right|$ where $\mathcal{F}^{*}=\mathcal{L}\left(X^{(k)},\left(\begin{array}{l}n \\ k\end{array}\right)+\right.$ $\left.\left(\begin{array}{l}n-1 \\ k-1\end{array}\right)\right) \cup\left[\mathcal{L}\left(X^{(k-1)}, t\right)+\{n+2\}\right]$.

Proof By Kruskal-Katona Theorem,

$$
\begin{gathered}
|\partial(\mathcal{F}, i \wedge j)| \geq\left(\begin{array}{c}
m_{k-1}-2 \\
k-3
\end{array}\right)+\left(\begin{array}{c}
m_{k-2}-3 \\
k-4
\end{array}\right)+\cdots+\left(\begin{array}{c}
m_{s+1}-2 \\
s-1
\end{array}\right)+\left(\begin{array}{c}
m_{s}-1 \\
s-2
\end{array}\right) \\
|\partial(\mathcal{F}, i \wedge \bar{j})| \geq\left|\mathcal{F}_{i} \cap \mathcal{F}_{j}\right| \geq \gamma(t) .
\end{gathered}
$$


Therefore

$$
\begin{aligned}
|\partial(\mathcal{F}, i)| \geq & |\partial(\mathcal{F}, i \wedge j)|+|\partial(\mathcal{F}, i \wedge \bar{j})| \\
\geq & {\left[\left(\begin{array}{c}
m_{k-1}-2 \\
k-3
\end{array}\right)+\left(\begin{array}{c}
m_{k-2}-3 \\
k-4
\end{array}\right)+\cdots+\left(\begin{array}{c}
m_{s+1}-2 \\
s-1
\end{array}\right)+\left(\begin{array}{c}
m_{s}-1 \\
s-2
\end{array}\right)\right] } \\
& +\left[\left(\begin{array}{c}
m_{k-1}-2 \\
k-2
\end{array}\right)+\left(\begin{array}{c}
m_{k-2}-3 \\
k-3
\end{array}\right)+\cdots+\left(\begin{array}{c}
m_{s+1}-2 \\
s
\end{array}\right)+\left(\begin{array}{c}
m_{s}-1 \\
s-1
\end{array}\right)\right] \\
= & \left(\begin{array}{c}
m_{k-1}-1 \\
k-2
\end{array}\right)+\left(\begin{array}{c}
m_{k-2}-2 \\
k-3
\end{array}\right)+\cdots+\left(\begin{array}{c}
m_{s+1}-1 \\
s
\end{array}\right)+\left(\begin{array}{c}
m_{s} \\
s-1
\end{array}\right) \\
= & \left|\partial \mathcal{L}\left(X^{(k-1)}, \alpha(t)\right)\right|
\end{aligned}
$$

On the other hand, $\left|\mathcal{F}_{\bar{i}}\right|>\left(\begin{array}{l}n \\ k\end{array}\right)+\left(\begin{array}{l}n-1 \\ k-1\end{array}\right)+\beta(t)$. By the Kruskal-Katona Theorem again,

$$
|\partial(\mathcal{F}, \bar{i})| \geq\left(\begin{array}{c}
n \\
k-1
\end{array}\right)+\left(\begin{array}{c}
n-1 \\
k-2
\end{array}\right)+\left|\partial \mathcal{L}\left(X^{(k-2)}, \beta(t)\right)\right|
$$

Therefore

$$
\begin{aligned}
|\partial \mathcal{F}| & =|\partial(\mathcal{F}, i)|+|\partial(\mathcal{F}, \bar{i})| \\
& \geq\left|\partial \mathcal{L}\left(X^{(k-1)}, \alpha(t)\right)\right|+\left(\begin{array}{c}
n \\
k-1
\end{array}\right)+\left(\begin{array}{c}
n-1 \\
k-2
\end{array}\right)+\left|\partial \mathcal{L}\left(X^{(k-2)}, \beta(t)\right)\right| \\
& \geq\left(\begin{array}{c}
n \\
k-1
\end{array}\right)+\left(\begin{array}{c}
n-1 \\
k-2
\end{array}\right)+\left|\partial \mathcal{L}\left(X^{(k-1)}, t\right)\right| \\
& =\left|\partial \mathcal{F}^{*}\right|
\end{aligned}
$$

The last inequality follows from Lemma 4.3.3.

Theorem 4.3.5. Let $X=[n+2], \mathcal{F} \subseteq X^{(k)}$ with $|\mathcal{F}|=\left(\begin{array}{l}n \\ k\end{array}\right)+\left(\begin{array}{l}n-1 \\ k-1\end{array}\right)+t, 0<t \leq\left(\begin{array}{l}n-1 \\ k-1\end{array}\right)$. If $\forall i \in$ $X, c(i) \leq\left(\begin{array}{l}n \\ k\end{array}\right)+\left(\begin{array}{c}n-1 \\ k-1\end{array}\right)$, then $|\partial \mathcal{F}| \geq\left|\partial \mathcal{F}^{*}\right|$, where $\mathcal{F}^{*}=\mathcal{L}\left(X^{(k)},\left(\begin{array}{l}n \\ k\end{array}\right)+\left(\begin{array}{l}n-1 \\ k-1\end{array}\right)\right) \cup\left[\mathcal{L}\left(X^{(k-1)}, t\right)+\{n+2\}\right]$. 
Proof By Lemma 4.3.3, we may assume $d(i)>\left(\begin{array}{c}n-1 \\ k-1\end{array}\right)+\beta(t) \forall i \in X$.

Because shifting never decreases the size of the shadow, we can apply shifting on $\mathcal{F}$. If at some stage, the degree of some vertex drops between $\alpha(t)$ and $\left(\begin{array}{l}n-1 \\ k-1\end{array}\right)+\beta(t)$, then by Lemma 4.3.3, we are done. If finally we obtain a left compressed set system that still satisfies the degree condition, then by Lemma 4.3.1, we are done. So we may assume at some stage when we shift $n+2$ to $n+1, d(n+2)$ drops from above $\left(\begin{array}{l}n-1 \\ k-1\end{array}\right)+\beta(t)$ to below $\alpha(t)$. Denote the set system before that step by $\mathcal{F}^{\prime}$ and the one after that step by $\mathcal{F}^{\prime \prime}$. So $d_{\mathcal{F}^{\prime \prime}}(n+2)<\alpha(t)$.

By Lemma 4.3.4, we can further assume that $\left|\mathcal{F}_{n+1, n+2}^{\prime \prime}\right|<\gamma(t)$, thus $\left|\mathcal{F}_{n+1, n+2}^{\prime}\right|<\gamma(t)$.

Now let's estimate the size of $\partial \mathcal{F}^{\prime}$.

Let $a_{0}=\mid\left\{A \in \mathcal{F}^{\prime}: n+1 \notin A\right.$ and $\left.n+2 \notin A\right\} \mid$,

$a_{1}=\mid\left\{A \in \mathcal{F}^{\prime}: n+1 \in A\right.$ and $\left.n+2 \notin A\right\} \mid$,

$a_{2}=\mid\left\{A \in \mathcal{F}^{\prime}: n+1 \notin A\right.$ and $\left.n+2 \in A\right\} \mid$,

$a_{12}=\mid\left\{A \in \mathcal{F}^{\prime}: n+1 \in A\right.$ and $\left.n+2 \in A\right\} \mid$.

Let

$$
\begin{aligned}
\mathcal{G}= & \mathcal{L}\left(X^{(k)}, a_{0}\right) \cup\left[\mathcal{L}\left(X^{(k-1)}, a_{1}+a_{2}-\left(\begin{array}{l}
n-1 \\
k-1
\end{array}\right)\right)+\{n+1\}\right] \\
& \cup\left[\mathcal{L}\left(X^{(k-1)},\left(\begin{array}{l}
n-1 \\
k-1
\end{array}\right)\right)+\{n+2\}\right] \cup\left[\mathcal{L}\left(X^{(k-2)}, a_{12}\right)+\{n+1, n+2\}\right]
\end{aligned}
$$

Then $|\mathcal{G}|=|\mathcal{F}|$, and a simple calculation shows that $a_{1}+a_{2}-\left(\begin{array}{c}n-1 \\ k-1\end{array}\right)>\left(\begin{array}{l}n-1 \\ k-1\end{array}\right)$.

Because $d_{\mathcal{F}^{\prime \prime}}(n+2)<\alpha(t)$, by the definition of shifting, we know $\left|\mathcal{F}_{n+1} \cap \mathcal{F}_{n+2}\right|<\alpha(t)$. On the other hand, $\left|\mathcal{G}_{n+1} \cap \mathcal{G}_{n+2}\right|=\left(\begin{array}{l}n-1 \\ k-1\end{array}\right)>\alpha(t)$. Therefore

$$
\left|\partial\left(\mathcal{F}^{\prime}, \overline{n+1} \wedge \overline{n+2}\right)\right| \geq|\partial(\mathcal{G}, \overline{n+1} \wedge \overline{n+2})|
$$

By the theorem in Section 4.2, $\left|\partial\left(\mathcal{F}^{\prime}, n+1 \vee n+2\right)\right| \geq|\partial(\mathcal{G}, n+1 \vee n+2)|$.

Therefore $\left|\partial \mathcal{F}^{\prime}\right| \geq|\partial \mathcal{G}|$. 
Now $d_{\mathcal{G}}(n+2)<\left(\begin{array}{c}n-1 \\ k-1\end{array}\right)+\gamma(t)<\left(\begin{array}{l}n-1 \\ k-1\end{array}\right)+\beta(t)$, and $d_{\mathcal{G}}(n+2)>\left(\begin{array}{l}n-1 \\ k-1\end{array}\right)>\alpha(t)$. By Lemma 4.3.3, $|\partial \mathcal{G}| \geq\left|\partial \mathcal{F}^{*}\right|$.

It follows that $\left|\partial \mathcal{F}^{\prime}\right| \geq\left|\partial \mathcal{F}^{*}\right|$.

Lemma 4.3.6. Let $X=[n+2], \mathcal{F} \subseteq X^{(k)}$ be a left compressed set system. $|\mathcal{F}|=\left(\begin{array}{l}n \\ k\end{array}\right)+2\left(\begin{array}{c}n-1 \\ k-1\end{array}\right)+t$, $0<t \leq\left(\begin{array}{l}n-2 \\ k-2\end{array}\right)$. If $\forall i \in X, c(i) \leq\left(\begin{array}{l}n \\ k\end{array}\right)+\left(\begin{array}{l}n-1 \\ k-1\end{array}\right)$, then $|\partial \mathcal{F}| \geq\left|\partial \mathcal{F}^{*}\right|$, where $\mathcal{F}^{*}=\mathcal{L}\left(X^{(k)},\left(\begin{array}{l}n \\ k\end{array}\right)+\right.$ $\left.\left(\begin{array}{c}n-1 \\ k-1\end{array}\right)+t\right) \cup\left[\mathcal{L}\left(X^{(k-1)},\left(\begin{array}{c}n-1 \\ k-1\end{array}\right)\right)+\{n+2\}\right]$.

Proof The same argument as Lemma 4.3.1.

Lemma 4.3.7. Let $X=[n+2], \mathcal{F} \subseteq X^{(k)}$ with $|\mathcal{F}|=\left(\begin{array}{l}n \\ k\end{array}\right)+2\left(\begin{array}{c}n-1 \\ k-1\end{array}\right)+t$ where $0<t \leq\left(\begin{array}{c}n-2 \\ k-2\end{array}\right)$. If $\exists i$ with $\left(\begin{array}{c}n-1 \\ k-1\end{array}\right) \leq d(i) \leq\left(\begin{array}{l}n-1 \\ k-1\end{array}\right)+\left(\begin{array}{c}n-2 \\ k-2\end{array}\right)$, then $|\partial \mathcal{F}| \geq\left|\partial \mathcal{F}^{*}\right|$ where $\mathcal{F}^{*}=\mathcal{L}\left(X^{(k)},\left(\begin{array}{l}n \\ k\end{array}\right)+\left(\begin{array}{l}n-1 \\ k-1\end{array}\right)+t\right) \cup$ $\left[\mathcal{L}\left(X^{(k-1)},\left(\begin{array}{l}n-1 \\ k-1\end{array}\right)\right)+\{n+2\}\right]$.

Proof By a similar argument to Lemma 4.3.3.

Lemma 4.3.8. Let $X=[n+2], \mathcal{F} \subseteq X^{(k)}$ with $|\mathcal{F}|=\left(\begin{array}{l}n \\ k\end{array}\right)+2\left(\begin{array}{c}n-1 \\ k-1\end{array}\right)+t$ where $0<t \leq$ $\left(\begin{array}{l}n-2 \\ k-2\end{array}\right)$. If $\exists i$ with $d(i)<\left(\begin{array}{l}n-1 \\ k-1\end{array}\right)$ and $\exists j$ with $\left|\mathcal{F}_{i} \cap \mathcal{F}_{j}\right| \geq\left(\begin{array}{c}n-2 \\ k-2\end{array}\right)$, then $|\partial \mathcal{F}| \geq\left|\partial \mathcal{F}^{*}\right|$ where $\mathcal{F}^{*}=\mathcal{L}\left(X^{(k)},\left(\begin{array}{l}n \\ k\end{array}\right)+\left(\begin{array}{l}n-1 \\ k-1\end{array}\right)+t\right) \cup\left[\mathcal{L}\left(X^{(k-1)},\left(\begin{array}{l}n-1 \\ k-1\end{array}\right)\right)+\{n+2\}\right]$.

Proof The same argument as Lemma 4.3.4.

Theorem 4.3.9. Let $X=[n+2], \mathcal{F} \subseteq X^{(k)}$ with $|\mathcal{F}|=\left(\begin{array}{l}n \\ k\end{array}\right)+2\left(\begin{array}{l}n-1 \\ k-1\end{array}\right)+t, 0<t \leq\left(\begin{array}{c}n-2 \\ k-2\end{array}\right)$. If $\forall i \in X, c(i) \leq\left(\begin{array}{l}n \\ k\end{array}\right)+\left(\begin{array}{l}n-1 \\ k-1\end{array}\right)$, then $|\partial \mathcal{F}| \geq\left|\partial \mathcal{F}^{*}\right|$, where $\mathcal{F}^{*}=\mathcal{L}\left(X^{(k)},\left(\begin{array}{l}n \\ k\end{array}\right)+\left(\begin{array}{l}n-1 \\ k-1\end{array}\right)+t\right) \cup$ $\left[\mathcal{L}\left(X^{(k-1)},\left(\begin{array}{l}n-1 \\ k-1\end{array}\right)\right)+\{n+2\}\right]$.

Proof By Lemma 4.3.7, we may assume $d(i)>\left(\begin{array}{l}n-1 \\ k-1\end{array}\right)+\left(\begin{array}{l}n-2 \\ k-2\end{array}\right) \forall i \in X$.

Because shifting never decreases the size of the shadow, we can apply shifting on $\mathcal{F}$. If at some stage, the degree of some vertex drops between $\left(\begin{array}{l}n-1 \\ k-1\end{array}\right)$ and $\left(\begin{array}{c}n-1 \\ k-1\end{array}\right)+\left(\begin{array}{l}n-2 \\ k-2\end{array}\right)$, then by Lemma 
4.3.7, we are done. If finally we obtain a left compressed set system that still satisfies the degree condition, then by Lemma 4.3.6, we are done. So we may assume at some stage when we shift $n+2$ to $n+1, d(n+2)$ drops from above $\left(\begin{array}{l}n-1 \\ k-1\end{array}\right)+\left(\begin{array}{l}n-2 \\ k-2\end{array}\right)$ to below $\left(\begin{array}{l}n-1 \\ k-1\end{array}\right)$. Denote the set system before that step by $\mathcal{F}^{\prime}$ and the one after that step by $\mathcal{F}^{\prime \prime}$. So $d_{\mathcal{F}^{\prime \prime}}(n+2)<\left(\begin{array}{l}n-1 \\ k-1\end{array}\right)$.

By Lemma 4.3.4, we can further assume that $\left|\mathcal{F}_{n+1, n+2}^{\prime \prime}\right|<\left(\begin{array}{l}n-2 \\ k-2\end{array}\right)$, thus $\left|\mathcal{F}_{n+1, n+2}^{\prime}\right|<\left(\begin{array}{c}n-2 \\ k-2\end{array}\right)$. Now let's estimate the size of $\partial \mathcal{F}^{\prime}$.

Let $a_{0}=\mid\left\{A \in \mathcal{F}^{\prime}: n+1 \notin A\right.$ and $\left.n+2 \notin A\right\} \mid$,

$a_{1}=\mid\left\{A \in \mathcal{F}^{\prime}: n+1 \in A\right.$ and $\left.n+2 \notin A\right\} \mid$,

$a_{2}=\mid\left\{A \in \mathcal{F}^{\prime}: n+1 \notin A\right.$ and $\left.n+2 \in A\right\} \mid$,

$a_{12}=\mid\left\{A \in \mathcal{F}^{\prime}: n+1 \in A\right.$ and $\left.n+2 \in A\right\} \mid$.

Let

$$
\begin{aligned}
\mathcal{G}= & \mathcal{L}\left(X^{(k)}, a_{0}\right) \cup\left[\mathcal{L}\left(X^{(k-1)}, a_{1}+a_{2}-\left(\begin{array}{l}
n-1 \\
k-1
\end{array}\right)\right)+\{n+1\}\right] \\
& \cup\left[\mathcal{L}\left(X^{(k-1)},\left(\begin{array}{l}
n-1 \\
k-1
\end{array}\right)\right)+\{n+2\}\right] \cup\left[\mathcal{L}\left(X^{(k-2)}, a_{12}\right)+\{n+1, n+2\}\right]
\end{aligned}
$$

Then $|\mathcal{G}|=|\mathcal{F}|$, and a simple calculation shows that $a_{1}+a_{2}-\left(\begin{array}{c}n-1 \\ k-1\end{array}\right)>\left(\begin{array}{l}n-1 \\ k-1\end{array}\right)$.

Because $d_{\mathcal{F}^{\prime \prime}}(n+2)<\left(\begin{array}{c}n-2 \\ k-2\end{array}\right)$, by the definition of shifting, we know $\left|\mathcal{F}_{n+1} \cap \mathcal{F}_{n+2}\right|<\left(\begin{array}{c}n-2 \\ k-2\end{array}\right)$. On the other hand, $\left|\mathcal{G}_{n+1} \cap \mathcal{G}_{n+2}\right|=\left(\begin{array}{c}n-1 \\ k-1\end{array}\right)>\left(\begin{array}{c}n-2 \\ k-2\end{array}\right)$. Therefore

$$
\left|\partial\left(\mathcal{F}^{\prime}, \overline{n+1} \wedge \overline{n+2}\right)\right| \geq|\partial(\mathcal{G}, \overline{n+1} \wedge \overline{n+2})| .
$$

By the theorem in Section 4.2,

$$
\left|\partial\left(\mathcal{F}^{\prime}, n+1 \vee n+2\right)\right| \geq|\partial(\mathcal{G}, n+1 \vee n+2)| .
$$

Therefore $\left|\partial \mathcal{F}^{\prime}\right| \geq|\partial \mathcal{G}|$. 
Now $d_{\mathcal{G}}(n+2)<\left(\begin{array}{l}n-1 \\ k-1\end{array}\right)+\left(\begin{array}{l}n-2 \\ k-2\end{array}\right)$, and $d_{\mathcal{G}}(n+2)>\left(\begin{array}{l}n-1 \\ k-1\end{array}\right)>\left(\begin{array}{l}n-2 \\ k-2\end{array}\right)$. By Lemma 4.3.7, $|\partial \mathcal{G}| \geq\left|\partial \mathcal{F}^{*}\right|$.

It follows that $\left|\partial \mathcal{F}^{\prime}\right| \geq\left|\partial \mathcal{F}^{*}\right|$.

Theorem 4.3.10. Let $X=[n+3], \mathcal{F} \subseteq X^{(k)}$ be a left compressed set system. $|\mathcal{F}|=\left(\begin{array}{l}n \\ k\end{array}\right)+$ $2\left(\begin{array}{c}n-1 \\ k-1\end{array}\right)+t, 0<t \leq\left(\begin{array}{c}n-1 \\ k-1\end{array}\right)$. If $\forall i, j \in X$ with $i \neq j, c(i) \leq\left(\begin{array}{l}n \\ k\end{array}\right)+2\left(\begin{array}{c}n-1 \\ k-1\end{array}\right)$, and $c(i, j) \leq\left(\begin{array}{l}n \\ k\end{array}\right)+$ $\left(\begin{array}{l}n-1 \\ k-1\end{array}\right)$, then $|\partial \mathcal{F}| \geq\left|\partial \mathcal{F}^{*}\right|$, where $\mathcal{F}^{*}=\mathcal{L}\left(X^{(k)},\left(\begin{array}{l}n \\ k\end{array}\right)+\left(\begin{array}{l}n-1 \\ k-1\end{array}\right)\right) \cup\left[\mathcal{L}\left(X^{(k-1)},\left(\begin{array}{c}n-1 \\ k-1\end{array}\right)\right)+\{n+2\}\right] \cup$ $\left[\mathcal{L}\left(X^{(k-1)}, t\right)+\{n+3\}\right]$.

Proof Use induction on $k$ and $|\mathcal{F}|$.

When $k=2,|\partial \mathcal{F}|=$ number of symbols used in $\mathcal{F}$. By the degree condition, we have $|\partial \mathcal{F}|=\left|\partial \mathcal{F}^{*}\right|$.

Assume the theorem is true for smaller $k$ and $|\mathcal{F}|$. Let $t=b^{(k-1)}\left(m_{k-1}, \cdots, m_{s}\right)$, then

$$
\begin{gathered}
\left|\partial \mathcal{F}^{*}\right|=\left(\begin{array}{c}
n \\
k-1
\end{array}\right)+2\left(\begin{array}{l}
n-1 \\
k-2
\end{array}\right)+b^{(k-2)}\left(m_{k-1}, \cdots, m_{s}\right), \\
|\mathcal{F}|=\left(\begin{array}{c}
n-1 \\
k-1
\end{array}\right)+2\left(\begin{array}{c}
n-2 \\
k-2
\end{array}\right)+b^{(k-2)}\left(m_{k-1}-1, \cdots, m_{s}-1\right) \\
\quad+\left(\begin{array}{c}
n-1 \\
k
\end{array}\right)+2\left(\begin{array}{c}
n-2 \\
k-1
\end{array}\right)+b^{(k-1)}\left(m_{k-1}-1, \cdots, m_{s}-1\right)
\end{gathered}
$$

Since $\mathcal{F}$ is left compressed, we have $\partial \mathcal{F}_{\overline{1}} \subseteq \mathcal{F}(1)$.

Case 1. $\left|\mathcal{F}_{\overline{1}}\right|>\left(\begin{array}{c}n-1 \\ k\end{array}\right)+2\left(\begin{array}{c}n-2 \\ k-1\end{array}\right)+b^{(k-1)}\left(m_{k-1}-1, \cdots, m_{s}-1\right)$

1(a). $\exists i$ and $j$ with $c_{\mathcal{F}_{\text {bar } 1}}(i, j)>\left(\begin{array}{c}n-1 \\ k\end{array}\right)+\left(\begin{array}{c}n-2 \\ k-1\end{array}\right)$.

By Kruskal-Katona Theorem, $\left|\partial \mathcal{F}_{\overline{1}, \bar{i}, \bar{j}}\right| \geq\left(\begin{array}{c}n-1 \\ k-1\end{array}\right)+\left(\begin{array}{c}n-2 \\ k-2\end{array}\right)$.

$$
\left|\mathcal{F}_{1, \bar{i}, \bar{j}}\right| \geq\left|\partial \mathcal{F}_{\overline{1}, \bar{i}, \bar{j}}\right| \geq\left(\begin{array}{l}
n-1 \\
k-1
\end{array}\right)+\left(\begin{array}{l}
n-2 \\
k-2
\end{array}\right)
$$


Thus $\left|\partial \mathcal{F}_{1, \bar{i}, \bar{j}}\right| \geq\left(\begin{array}{c}n-1 \\ k-2\end{array}\right)+\left(\begin{array}{c}n-2 \\ k-3\end{array}\right)$ by Kruskal-Katona Theorem.

Therefore $\left|\partial \mathcal{F}_{\bar{i} . \bar{j}}\right| \geq\left(\begin{array}{l}n-1 \\ k-1\end{array}\right)+\left(\begin{array}{c}n-2 \\ k-2\end{array}\right)+\left(\begin{array}{c}n-1 \\ k-2\end{array}\right)+\left(\begin{array}{c}n-2 \\ k-3\end{array}\right)=\left(\begin{array}{c}n \\ k-1\end{array}\right)+\left(\begin{array}{c}n-1 \\ k-2\end{array}\right)$.

By the degree condition, we can prove that

$$
|\partial(\mathcal{F}, i \vee j)| \geq\left(\begin{array}{c}
n-1 \\
k-2
\end{array}\right)+b^{(k-2)}\left(m_{k-1}, \cdots, m_{s}\right)
$$

Therefore $|\partial \mathcal{F}| \geq\left(\begin{array}{c}n \\ k-1\end{array}\right)+\left(\begin{array}{c}n-1 \\ k-2\end{array}\right)+\left(\begin{array}{c}n-1 \\ k-2\end{array}\right)+b^{(k-2)}\left(m_{k-1}, \cdots, m_{s}\right)=\left|\partial \mathcal{F}^{*}\right|$.

1(b) $\exists i$ with $c_{\mathcal{F}_{\overline{1}}}(i)>\left(\begin{array}{c}n-1 \\ k\end{array}\right)+2\left(\begin{array}{c}n-2 \\ k-1\end{array}\right)$ but don't exist $i$ and $j$ in $1(\mathrm{a})$.

By Theorem 4.3.5, $\left|\partial\left(\mathcal{F}_{\overline{1}}, \bar{i}\right)\right| \geq\left(\begin{array}{c}n-1 \\ k-1\end{array}\right)+2\left(\begin{array}{c}n-2 \\ k-2\end{array}\right)$.

$\left|\mathcal{F}_{1, \bar{i}}\right| \geq\left|\partial\left(\mathcal{F}_{\overline{1}}, \bar{i}\right)\right| \geq\left(\begin{array}{c}n-1 \\ k-1\end{array}\right)+2\left(\begin{array}{c}n-2 \\ k-2\end{array}\right)$.

Therefore

$$
c_{\mathcal{F}}(i)=c_{\mathcal{F}_{\overline{1}}}(i)+\left|\mathcal{F}_{1, \bar{i}}\right|>\left(\begin{array}{c}
n-1 \\
k
\end{array}\right)+2\left(\begin{array}{l}
n-2 \\
k-1
\end{array}\right)+\left(\begin{array}{l}
n-1 \\
k-1
\end{array}\right)+2\left(\begin{array}{l}
n-2 \\
k-2
\end{array}\right)=\left(\begin{array}{l}
n \\
k
\end{array}\right)+2\left(\begin{array}{l}
n-1 \\
k-1
\end{array}\right) .
$$

It's a contradiction to the degree condition.

1 (c) $\forall i, j \in X$ with $i \neq j, c_{\mathcal{F}_{\overline{1}}}(i) \leq\left(\begin{array}{c}n-1 \\ k\end{array}\right)+2\left(\begin{array}{c}n-2 \\ k-1\end{array}\right)$, and $c_{\mathcal{F}_{\overline{1}}}(i, j) \leq\left(\begin{array}{c}n-1 \\ k\end{array}\right)+\left(\begin{array}{c}n-2 \\ k-1\end{array}\right)$

Then by inductive hypothesis,

$$
\left|\partial \mathcal{F}_{\overline{1}}\right| \geq\left(\begin{array}{l}
n-1 \\
k-1
\end{array}\right)+2\left(\begin{array}{l}
n-2 \\
k-2
\end{array}\right)+b^{(k-2)}\left(m_{k-1}-1, \cdots, m_{s}-1\right) .
$$


Therefore

$$
\begin{aligned}
|\mathcal{F}|= & \left|\mathcal{F}_{1}\right|+\left|\mathcal{F}_{\overline{1}}\right| \\
\geq & \left|\partial \mathcal{F}_{\overline{1}}\right|+\left|\mathcal{F}_{\overline{1}}\right| \\
> & \left(\begin{array}{c}
n-1 \\
k-1
\end{array}\right)+2\left(\begin{array}{l}
n-2 \\
k-2
\end{array}\right)+b^{(k-2)}\left(m_{k-1}-1, \cdots, m_{s}-1\right) \\
& +\left(\begin{array}{c}
n-1 \\
k
\end{array}\right)+2\left(\begin{array}{l}
n-2 \\
k-1
\end{array}\right)+b^{(k-1)}\left(m_{k-1}-1, \cdots, m_{s}-1\right) \\
= & \left(\begin{array}{l}
n \\
k
\end{array}\right)+2\left(\begin{array}{l}
n-1 \\
k-1
\end{array}\right)+b^{(k-1)}\left(m_{k-1}, \cdots, m_{s}\right)
\end{aligned}
$$

contradicting the assumption on $|\mathcal{F}|$.

Case 2. $\left|\mathcal{F}_{\overline{1}}\right| \leq\left(\begin{array}{c}n-1 \\ k\end{array}\right)+2\left(\begin{array}{c}n-2 \\ k-1\end{array}\right)+b^{(k-1)}\left(m_{k-1}-1, \cdots, m_{s}-1\right)$

then $\left|\mathcal{F}_{1}\right| \geq\left(\begin{array}{c}n-1 \\ k-1\end{array}\right)+2\left(\begin{array}{c}n-2 \\ k-2\end{array}\right)+b^{(k-2)}\left(m_{k-1}-1, \cdots, m_{s}-1\right)$.

2(a) $\exists i$ and $j$ with $c_{\mathcal{F}_{1}}(i, j)>\left(\begin{array}{l}n-1 \\ k-1\end{array}\right)+\left(\begin{array}{l}n-2 \\ k-2\end{array}\right)$.

By a similar argument to $1(\mathrm{a})$, we can show $|\partial \mathcal{F}| \geq\left|\partial \mathcal{F}^{*}\right|$.

2(b) $\exists i$ with $c_{\mathcal{F}_{1}}(i)>\left(\begin{array}{c}n-1 \\ k-1\end{array}\right)+2\left(\begin{array}{c}n-2 \\ k-2\end{array}\right)$ but don't exist $i$ and $j$ in $2($ a).

Then by Theorem 4.3.5, $|\partial(\mathcal{F}(1), \bar{i})| \geq\left(\begin{array}{c}n-1 \\ k-2\end{array}\right)+2\left(\begin{array}{c}n-2 \\ k-3\end{array}\right)$.

So

$$
\begin{aligned}
\left|\partial \mathcal{F}_{1, \bar{i}}\right| & \geq\left|\mathcal{F}_{1, \bar{i}}\right|+|\partial(\mathcal{F}(1), \bar{i})| \\
& \geq\left(\begin{array}{l}
n-1 \\
k-1
\end{array}\right)+2\left(\begin{array}{l}
n-2 \\
k-2
\end{array}\right)+\left(\begin{array}{l}
n-1 \\
k-2
\end{array}\right)+2\left(\begin{array}{l}
n-2 \\
k-3
\end{array}\right) \\
& =\left(\begin{array}{c}
n \\
k-1
\end{array}\right)+2\left(\begin{array}{l}
n-1 \\
k-2
\end{array}\right) .
\end{aligned}
$$


On the other hand, by the degree condition on $i$ and Kruskal-Katona Theorem, we have

$$
|\partial(\mathcal{F}, i)| \geq b^{(k-2)}\left(m_{k-1}, \cdots, m_{s}\right)
$$

Therefore

$$
|\partial \mathcal{F}| \geq\left|\partial \mathcal{F}_{1, \bar{i}}\right|+|\partial(\mathcal{F}, i)| \geq\left(\begin{array}{c}
n \\
k-1
\end{array}\right)+2\left(\begin{array}{c}
n-1 \\
k-2
\end{array}\right)+b^{(k-2)}\left(m_{k-1}, \cdots, m_{s}\right)=\left|\partial \mathcal{F}^{*}\right|
$$

2(c) $\forall i, j \in X$ with $i \neq j, c_{\mathcal{F}_{1}}(i) \leq\left(\begin{array}{c}n-1 \\ k-1\end{array}\right)+2\left(\begin{array}{c}n-2 \\ k-2\end{array}\right)$, and $c_{\mathcal{F}_{1}}(i, j) \leq\left(\begin{array}{c}n-1 \\ k-1\end{array}\right)+\left(\begin{array}{c}n-2 \\ k-2\end{array}\right)$

Then by inductive hypothesis,

$$
|\partial \mathcal{F}(1)| \geq\left(\begin{array}{l}
n-1 \\
k-2
\end{array}\right)+2\left(\begin{array}{l}
n-2 \\
k-3
\end{array}\right)+b^{(k-3)}\left(m_{k-1}-1, \cdots, m_{s}-1\right) .
$$

Thus

$$
\begin{aligned}
\left|\partial \mathcal{F}_{1}\right| \geq & \left|\mathcal{F}_{1}\right|+|\partial \mathcal{F}(1)| \\
\geq & \left(\begin{array}{l}
n-1 \\
k-1
\end{array}\right)+2\left(\begin{array}{l}
n-2 \\
k-2
\end{array}\right)+b^{(k-2)}\left(m_{k-1}-1, \cdots, m_{s}-1\right) \\
& +\left(\begin{array}{l}
n-1 \\
k-2
\end{array}\right)+2\left(\begin{array}{l}
n-2 \\
k-3
\end{array}\right)+b^{(k-3)}\left(m_{k-1}-1, \cdots, m_{s}-1\right) \\
= & \left(\begin{array}{c}
n \\
k-1
\end{array}\right)+2\left(\begin{array}{l}
n-1 \\
k-2
\end{array}\right)+b^{(k-2)}\left(m_{k-1}, \cdots, m_{s}\right) \\
= & \left|\partial \mathcal{F}^{*}\right|
\end{aligned}
$$

Lemma 4.3.11. Let $X=[n+3], \mathcal{F} \subseteq X^{(k)}$ with $|\mathcal{F}|=\left(\begin{array}{c}n \\ k\end{array}\right)+2\left(\begin{array}{c}n-1 \\ k-1\end{array}\right)+t$ where $0<t \leq\left(\begin{array}{c}n-1 \\ k-1\end{array}\right)$. If $\exists i$ with $\alpha(t) \leq d(i) \leq\left(\begin{array}{c}n-1 \\ k-1\end{array}\right)+\beta(t)$, and $\forall j_{1}, j_{2}$ other than $i, c\left(j_{1}, j_{2}\right) \leq\left(\begin{array}{l}n \\ k\end{array}\right)+\left(\begin{array}{c}n-1 \\ k-1\end{array}\right)$, then $|\partial \mathcal{F}| \geq$ $\left|\partial \mathcal{F}^{*}\right|$ where $\mathcal{F}^{*}=\mathcal{L}\left(X^{(k)},\left(\begin{array}{l}n \\ k\end{array}\right)+\left(\begin{array}{c}n-1 \\ k-1\end{array}\right)\right) \cup\left[\mathcal{L}\left(X^{(k-1)},\left(\begin{array}{c}n-1 \\ k-1\end{array}\right)\right)+\{n+2\}\right] \cup\left[\mathcal{L}\left(X^{(k-1)}, t\right)+\{n+3\}\right]$. 
Proof Case 1. $\alpha(t) \leq d(i)<t$.

Then $c(i)>\left(\begin{array}{l}n \\ k\end{array}\right)+2\left(\begin{array}{l}n-1 \\ k-1\end{array}\right)$. Let $a=c(i)-\left(\begin{array}{l}n \\ k\end{array}\right)-2\left(\begin{array}{l}n-1 \\ k-1\end{array}\right)$.

By Theorem 4.3.9, $|\partial(\mathcal{F}, \bar{i})| \geq\left(\begin{array}{c}n \\ k-1\end{array}\right)+2\left(\begin{array}{c}n-1 \\ k-2\end{array}\right)+\left|\partial \mathcal{L}\left(X^{(k-2)}, a\right)\right|$.

On the other hand, $\mid \partial(\mathcal{F}, i)) \geq\left|\partial \mathcal{L}\left(X^{(k-1)}, d(i)\right)\right|$.

Thus, $|\partial \mathcal{F}| \geq\left(\begin{array}{c}n \\ k-1\end{array}\right)+2\left(\begin{array}{c}n-1 \\ k-2\end{array}\right)+\left|\partial \mathcal{L}\left(X^{(k-2)}, a\right)\right|+\left|\partial \mathcal{L}\left(X^{(k-1)}, d(i)\right)\right|$.

Therefore $|\partial \mathcal{F}| \geq\left|\partial \mathcal{F}^{*}\right|$ is equivalent to

$\left(\begin{array}{c}n \\ k-1\end{array}\right)+2\left(\begin{array}{c}n-1 \\ k-2\end{array}\right)+\left|\partial \mathcal{L}\left(X^{(k-2)}, a\right)\right|+\left|\partial \mathcal{L}\left(X^{(k-1)}, d(i)\right)\right| \geq\left(\begin{array}{c}n \\ k-1\end{array}\right)+\left(\begin{array}{c}n-1 \\ k-2\end{array}\right)+\left|\partial \mathcal{L}\left(X^{(k-1)}, t\right)\right|$

This is what Lemma 4.3.3 says.

Case 2. $t \leq d(i) \leq\left(\begin{array}{l}n-1 \\ k-1\end{array}\right)+\beta(t)$.

Similar To Lemma 4.3.3.

Lemma 4.3.12. Let $X=[n+3], \mathcal{F} \subseteq X^{(k)}$ with $|\mathcal{F}|=\left(\begin{array}{l}n \\ k\end{array}\right)+2\left(\begin{array}{c}n-1 \\ k-1\end{array}\right)+t$ where $0<t \leq\left(\begin{array}{c}n-1 \\ k-1\end{array}\right)$. If $\exists i$ with $d(i)<\alpha(t)$ and $\exists j$ with $\left|\mathcal{F}_{i} \cap \mathcal{F}_{j}\right| \geq \gamma(t)$, and $c\left(j_{1}, j_{2}\right) \leq\left(\begin{array}{l}n \\ k\end{array}\right)+\left(\begin{array}{c}n-1 \\ k-1\end{array}\right) \forall j_{1}, j_{2}$ other than $i$, then $|\partial \mathcal{F}| \geq\left|\partial \mathcal{F}^{*}\right|$ where $\mathcal{F}^{*}=\mathcal{L}\left(X^{(k)},\left(\begin{array}{l}n \\ k\end{array}\right)+\left(\begin{array}{c}n-1 \\ k-1\end{array}\right)\right) \cup\left[\mathcal{L}\left(X^{(k-1)},\left(\begin{array}{c}n-1 \\ k-1\end{array}\right)\right)+\{n+2\}\right] \cup$ $\left[\mathcal{L}\left(X^{(k-1)}, t\right)+\{n+3\}\right]$.

Proof By the same argument as Lemma 4.3.4, we have $|\partial(\mathcal{F}, i)| \geq\left|\partial \mathcal{L}\left(X^{(k-1)}, \alpha(t)\right)\right|$.

On the other hand, $\left|\mathcal{F}_{\bar{i}}\right|>\left(\begin{array}{l}n \\ k\end{array}\right)+2\left(\begin{array}{c}n-1 \\ k-1\end{array}\right)+\beta(t)$.

By Theorem 4.3.9, $|\partial(\mathcal{F}, \bar{i})| \geq\left(\begin{array}{c}n \\ k-1\end{array}\right)+2\left(\begin{array}{c}n-1 \\ k-2\end{array}\right)+\left|\partial \mathcal{L}\left(X^{(k-2)}, \beta(t)\right)\right|$.

The rest of the proof is the same as Lemma 4.3.4.

Theorem 4.3.13. Let $X=[n+3], \mathcal{F} \subseteq X^{(k)}$ with $|\mathcal{F}|=\left(\begin{array}{l}n \\ k\end{array}\right)+2\left(\begin{array}{c}n-1 \\ k-1\end{array}\right)+t, 0<t \leq\left(\begin{array}{l}n-1 \\ k-1\end{array}\right)$. If 
$\forall i, j \in X$ with $i \neq j, c(i) \leq\left(\begin{array}{l}n \\ k\end{array}\right)+2\left(\begin{array}{l}n-1 \\ k-1\end{array}\right)$, and $c(i, j) \leq\left(\begin{array}{l}n \\ k\end{array}\right)+\left(\begin{array}{l}n-1 \\ k-1\end{array}\right)$, then $|\partial \mathcal{F}| \geq\left|\partial \mathcal{F}^{*}\right|$, where $\mathcal{F}^{*}=\mathcal{L}\left(X^{(k)},\left(\begin{array}{l}n \\ k\end{array}\right)+\left(\begin{array}{c}n-1 \\ k-1\end{array}\right)\right) \cup\left[\mathcal{L}\left(X^{(k-1)},\left(\begin{array}{l}n-1 \\ k-1\end{array}\right)\right)+\{n+2\}\right] \cup\left[\mathcal{L}\left(X^{(k-1)}, t\right)+\{n+3\}\right]$.

Proof By Lemma 4.3.11, we may assume $d(i)>\left(\begin{array}{l}n-1 \\ k-1\end{array}\right)+\beta(t) \forall i \in X$.

Because shifting never decreases the size of the shadow, we can apply shifting on $\mathcal{F}$. If at some stage, the degree of some vertex $i$ drops between $\alpha(t)$ and $\left(\begin{array}{c}n-1 \\ k-1\end{array}\right)+\beta(t)$, obviously we still

have $c\left(j_{1}, j_{2}\right) \leq\left(\begin{array}{l}n \\ k\end{array}\right)+\left(\begin{array}{l}n-1 \\ k-1\end{array}\right) \forall j_{1}, j_{2}$ other than $i$, thus by Lemma 4.3 .11 , we are done. If finally we obtain a left compressed set system that still satisfies the degree condition, then by Theorem 4.3.10, we are done. So we may assume at some stage when we shift $n+3$ to $n+2, d(n+3)$ drops from above $\left(\begin{array}{c}n-1 \\ k-1\end{array}\right)+\beta(t)$ to below $\alpha(t)$. Denote the set system before that step by $\mathcal{F}^{\prime}$ and the one after that step by $\mathcal{F}^{\prime \prime}$. So $d_{\mathcal{F}^{\prime \prime}}(n+3)<\alpha(t)$.

By the same argument as Theorem 4.3.5, we can show $\left|\partial \mathcal{F}^{\prime}\right| \geq\left|\partial \mathcal{F}^{*}\right|$.

\subsection{Intersecting set systems with degree conditions}

In this section we will study the following problem:

Problem. Let $n, k$ be two positive integers satisfying $n>2 k$ and let $X=[1, n]$. Find $\max |\mathcal{F}|$ where $\mathcal{F} \subseteq X^{(k)}$ is intersecting and $c(\mathcal{F})=s$ for a specified value $s$.

Without loss of generality, we may assume the point 1 has the minimum complementary degree. So $\left|\mathcal{F}_{\overline{1}}\right|=s$. Let $Y=[2, n]$, and let $\mathcal{A}=\left\{Y \backslash F: F \in \mathcal{F}_{\overline{1}}\right\}$. Then $|\mathcal{A}|=s$ and the size of a member of $\mathcal{A}$ is $r=n-1-k$.

Pick $F \in \mathcal{F}_{1}$. Because $\mathcal{F}$ is intersecting, $F \backslash\{1\} \notin \partial^{(k-1)} \mathcal{A}$. On the other hand, $\mathcal{F}$ is completely determined by $\mathcal{A}$ by the following operation: 
Definition 4.4.1. For $\mathcal{A} \subseteq Y^{(n-k-1)}$, define

$$
\begin{aligned}
\sigma_{0}(\mathcal{A}) & =\{Y \backslash F: F \in \mathcal{A}\}, \\
\sigma_{1}(\mathcal{A}) & =\left\{F \in X^{(k)}: 1 \in F\right\} \backslash\left(\partial^{(k-1)} \mathcal{A}+\{1\}\right) \\
& =\left\{F \in X^{(k)}: 1 \in F \text { and } F \cap B \neq \emptyset \text { for each } B \in \sigma_{0}(\mathcal{A}),\right. \\
\sigma(\mathcal{A}) & =\sigma_{0}(\mathcal{A}) \cup \sigma_{1}(\mathcal{A})
\end{aligned}
$$

We say that the set systems $\sigma(\mathcal{A}) \subseteq X^{(k)}$ and $\mathcal{A} \subseteq Y^{(n-k-1)}$ are associated. Thus the problem becomes finding the minimum $(k-1)$-shadow of $\mathcal{A}$ with $|\mathcal{A}|$ fixed and with the constraint on the minimum complementary degree.

Because we are looking at the complement of $\mathcal{F}_{\overline{1}}$ in $Y$, it's convenient to use the reverse of the natural order on $2,3, \cdots, n$.

Definition 4.4.2. Define "§" on $Y^{(r)}$ to be the COLEX order with respect to the reverse of the natural order on $2,3, \cdots, n$.

Let $\mathcal{F}_{(3,2)}=\left\{F \in X^{(k)}:|F \cap\{1,2,3\}| \geq 2\right\}$, and let $c(3,2)=c\left(\mathcal{F}_{(3,2)}\right)=\left(\begin{array}{l}n-3 \\ k-2\end{array}\right)$.

For $s \leq c(3,2)$, by the Kruskal-Katona Theorem, it is easy to see that $\sigma\left(\mathcal{L}\left(\preceq, Y^{(r)}, s\right)\right.$ is an optimal configuration to the above problem. In fact Goldwasser [9] proved a stronger theorem considering $c(\mathcal{F}) \geq s$ instead of $c(\mathcal{F})=s$ for $s \leq c(3,2)$. So we will study the case when $s>c(3,2)$.

Let $\mathcal{F}_{(5,3)}=\left\{F \in X^{(k)}:|F \cap\{1,2,3,4,5\}| \geq 3\right\}$, and let $c(5,3)=c\left(\mathcal{F}_{(5,3)}\right)=4\left(\begin{array}{c}n-5 \\ k-3\end{array}\right)+\left(\begin{array}{c}n-5 \\ k-4\end{array}\right)$. Simple calculation shows that $c(3,2)<c(5,3)$ if and only if $n<3 k-2$.

In this section we will study the case where $n<3 k-2$ and $c(3,2)<s \leq c(5,3)$.

Let's look at an example.

In order to describe a candidate configuration, we need to introduce some notation. The notation $\left[b_{1}, b_{p}\right] a_{1} a_{2} \cdots a_{q}$ where $b_{1}>b_{p}$ denotes a collection of subsets of $Y$. A set $A \subseteq Y$ belongs to this collection if and only if

(1) $\left\{a_{1}, a_{2}, \cdots, a_{q}\right\} \subseteq A$;

(2) $A \backslash\left\{a_{1}, a_{2} \cdots, a_{q}\right\} \subseteq\left[b_{p}, b_{1}\right]$;

(3) $|A|=r$. 
Example $n=15, k=6, r=8, s=525$.

Now $[15,9] 863$ means $[9,15]^{(5)}+\{8,6,3\}$.

We are ready to state to describe two families $\mathcal{A}_{1}, \mathcal{A}_{2} \subseteq[2,15]^{(8)}$ such that $\left|\mathcal{A}_{1}\right|=\left|\mathcal{A}_{2}\right|=525$ and $\left|\partial^{(5)} \mathcal{A}_{1}\right|=\left|\partial^{(5)} \mathcal{A}_{2}\right|=1092$. The associated set systems $\sigma\left(\mathcal{A}_{1}\right), \sigma\left(\mathcal{A}_{2}\right) \subseteq[15]^{(6)}$ each have size

$$
\left|\mathcal{A}_{1}\right|+\left[\left(\begin{array}{c}
14 \\
5
\end{array}\right)-\left|\partial^{(5)} \mathcal{A}_{1}\right|\right]=525+\left(\begin{array}{c}
14 \\
5
\end{array}\right)-1092=1435
$$

and minimum complementary degree $c\left(\sigma\left(\mathcal{A}_{1}\right)\right)=c\left(\sigma\left(\mathcal{A}_{2}\right)\right)=525$.

Example 1a. Let $\mathcal{A}_{1}$ be the union of:

$[15,6] 4$

$[15,6] 3$

$[15,6] 2$

It is not hard to see that the associated set system $\mathcal{F}_{1} \subseteq[15]^{(6)}$ is equal to $\mathcal{F}_{(5,3)}$.

Example 1b. Let $\mathcal{A}_{2}$ be the union of:

$$
\begin{aligned}
& {[15,6]} \\
& {[15,6] 5} \\
& {[15,6] 4} \\
& {[15,7] 54} \\
& {[15,7] 3} \\
& {[15,7] 2} \\
& {[15,7] 32}
\end{aligned}
$$

Definition 4.4.3. Let $\mathcal{A} \subseteq Y^{(r)}$. Define

$\mathcal{V}_{0}(\mathcal{A})=\{A \in \mathcal{A}: 3 \notin A$ and $2 \notin A\}$,

$\mathcal{V}_{3}(\mathcal{A})=\{A \in \mathcal{A}: 3 \in A$ and $2 \notin A\}$, 
$\mathcal{V}_{2}(\mathcal{A})=\{A \in \mathcal{A}: 3 \notin A$ and $2 \in A\}$,

$\mathcal{V}_{32}(\mathcal{A})=\{A \in \mathcal{A}: 3 \in A$ and $2 \in A\}$.

The last set (in COLEX order with symbols in reverse order) in $\mathcal{V}_{0}\left(\mathcal{A}_{2}\right)$ is $\{12,11,10,9,8,7,5,4\}$. It can be shown that if we add one more subset to $\mathcal{V}_{3}\left(\mathcal{A}_{2}\right)$ or $\mathcal{V}_{2}\left(\mathcal{A}_{2}\right)$ or $\mathcal{V}_{32}\left(\mathcal{A}_{2}\right)$, then the point 1 will no longer have minimum complementary degree in $\sigma\left(\mathcal{A}_{2}\right)$. Therefore the last member in $\mathcal{V}_{0}\left(\mathcal{A}_{2}\right)$ completely determines the last members in $\mathcal{V}_{3}\left(\mathcal{A}_{2}\right), \mathcal{V}_{2}\left(\mathcal{A}_{2}\right)$ and $\mathcal{V}_{32}\left(\mathcal{A}_{2}\right)$, something like conjugates.

To describe this conjugation, we need to introduce some definitions.

Definition 4.4.4. For $A \in Y^{(r)}$, a block of $A$ is a maximal subset of consecutive numbers of $A$.

For example, $\{5,4\}$ is a block of $\{12,11,10,9,8,7,5,4\}$.

Definition 4.4.5. A block of $A$ is the last block if it contains the largest number of $A$ (under natural order of symbols). The last block of $A$ is denoted by $B(A)$.

For example, $B(\{12,11,10,9,8,7,5,4\})=\{12,11,10,9,8,7\}$.

Note: The last block corresponds to the last term of the cascade form: if $\min B(A)=n-j$ and $|B(A)|=t$ then the last term in the cascade representation of the index of $A$ in COLEX is $\left(\begin{array}{c}j+1 \\ t\end{array}\right)$.

Definition 4.4.6. Let $A \in Y^{(r)}$. We say $A$ is regular if

(1) $\{5,4\} \subseteq A,\{3,2\} \cap A=\emptyset$,

(2) $|B(A)| \geq 2 r-n+3$.

Definition 4.4.7. Let $A \in Y^{(r)}$ be regular. Define

$$
C=[4, \min B(A)-2] \backslash(A \backslash B(A)),
$$

(Note that $|C|=\min B(A)-5-|A|+|B(A)|)$

$$
\begin{aligned}
& \operatorname{conj}_{1}(A)=C \cup\{\min B(A), \min B(A)+1, \cdots, \min B(A)+r-|C|-2\}, \\
& \operatorname{conj}_{2}(A)=C \cup\{\min B(A), \min B(A)+1, \cdots, \min B(A)+r-|C|-3\} .
\end{aligned}
$$


Note: Property (2) of regularity guarantees that $\operatorname{conj}_{1}(A)$ and $\operatorname{conj}_{2}(A)$ are well defined, and that $\left|\operatorname{conj}_{1}(A)\right|=\left|\operatorname{conj}_{2}(A)\right|+1=r-1$.

For example, for $n=13, r=8, A=\{12,11,10,9,8,7,5,4\}$, $\operatorname{conj}_{1}(A)=\{14,13,12,10,9,8,7\}, \operatorname{conj}_{2}(A)=\{13,12,10,9,8,7\}$.

Clearly, for the above example, the last members in $\mathcal{V}_{3}(\mathcal{A}), \mathcal{V}_{2}(\mathcal{A})$ and $\mathcal{V}_{32}(\mathcal{A})$ are $\operatorname{conj}_{1}(A) \cup$ $\{3\} \operatorname{conj}_{1}(A) \cup\{2\}$ and $\operatorname{conj}_{2}(A) \cup\{3,2\}$ respectively, and this is true in general.

Now with the conjugation notation, the last member in $\mathcal{V}_{0}(\mathcal{A})$ completely determines $\mathcal{A}$. Therefore we have the following definition:

Definition 4.4.8. Let $A \in Y^{(r)}$ be regular. Define

$\mathcal{U}_{0}(A)=\left\{F \in Y^{(r)}: F \preceq A\right\}$

$\mathcal{U}_{3}(A)=\left\{F \in Y^{(r-1)}: F \preceq \operatorname{conj}_{1}(A)\right\}+\{3\}$

$\mathcal{U}_{2}(A)=\left\{F \in Y^{(r-1)}: F \preceq \operatorname{conj}_{1}(A)\right\}+\{2\}$

$\mathcal{U}_{32}(A)=\left\{F \in Y^{(r-2)}: F \preceq \operatorname{conj}_{2}(A)\right\}+\{3,2\}$

$\mathcal{U}(A)=\mathcal{U}_{0}(A) \cup \mathcal{U}_{3}(A) \cup \mathcal{U}_{2}(A) \cup \mathcal{U}_{32}(A)$.

In the above example, we have $\left|\mathcal{A}_{1}\right|=\left|\mathcal{A}_{2}\right|=525$. If we want $s=456$ we need to take out 69 sets. We will show later that the best way to do so is take out the last 69 sets in $[15,7] 32$. The resulting set system is:

$[15,6] 4$

$[15,7] 54$

$[15,7] 3$

$[15,7] 2$

$[15,9] 32$

$[15,10] 832$

$[15,12] 9832$

$[15,13] 119832$

The size of the 5 -shadow of this set system is

$$
\left(\begin{array}{c}
10 \\
5
\end{array}\right)+\left(\begin{array}{c}
10 \\
4
\end{array}\right)+\left(\begin{array}{c}
10 \\
4
\end{array}\right)+\left(\begin{array}{l}
9 \\
3
\end{array}\right)+\left(\begin{array}{l}
9 \\
4
\end{array}\right)+\left(\begin{array}{l}
9 \\
4
\end{array}\right)+\left(\begin{array}{l}
7 \\
3
\end{array}\right)+\left(\begin{array}{l}
6 \\
2
\end{array}\right)+\left(\begin{array}{l}
4 \\
1
\end{array}\right)+\left(\begin{array}{l}
3 \\
0
\end{array}\right)=1063
$$


Now let's work out some rules on how to take out some sets from $\mathcal{U}(A)$.

Lemma 4.4.9. If $A \in Y^{(r)}$ is regular, then $\sigma(\mathcal{U}(A)) \subseteq X(k)$, and $\sigma(\mathcal{U}(A))$ is intersecting.

Proof The first part is trivial. For the second part, let $F_{1}, F_{2} \in \sigma(\mathcal{U}(A))$.

Case 1. $F_{1}, F_{2} \in \sigma_{1}(\mathcal{U}(A))$

Then $\{1\} \subseteq F_{1} \cap F_{2}$.

Case 2. $F_{1} \in \sigma_{0}(\mathcal{U}(A)), F_{2} \in \sigma_{1}(\mathcal{U}(A))$

If $F_{1} \cap F_{2}=\emptyset$, then $F_{2} \backslash\{1\} \in \partial^{(k-1)} \mathcal{U}(A)$, contradicting the definition of $\sigma_{1}(\mathcal{U}(A))$.

Case 3. $F_{1}, F_{2} \in \sigma_{0}(\mathcal{U}(A))$

Let $G_{1}=Y \backslash F_{1}, G_{2}=Y \backslash F_{2}$. Then $G_{1}, G_{2} \in \mathcal{U}(A)$ and $F_{1} \cap F_{2} \neq \emptyset$ is equivalent to $G_{1} \cup G_{2} \neq Y$. If $G_{1}, G_{2} \in \mathcal{U}_{0}(A)$, then $2 \notin G_{1}, 2 \notin G_{2}$. So $G_{1} \cup G_{2} \neq Y$. If $G_{1} \notin \mathcal{U}_{0}(A)$, $G_{2} \notin \mathcal{U}_{0}(A)$, then $4 \notin G_{1}, 4 \notin G_{2}$. If $G_{1} \in \mathcal{U}_{0}(A), G_{2} \notin \mathcal{U}_{0}(A)$, without loss of generality, we may assume $G_{2} \in \mathcal{U}_{3} 2(A)$. By the cross-intersecting property, we can further assume that $G_{1}=\max \mathcal{U}_{0}(A), G_{2}=\max \mathcal{U}_{32}(A)=\operatorname{conj}_{2}(A)+\{3,2\}$. But then $\min B(A)-1 \notin G_{1}$ and $\min B(A)-1 \notin G_{2}$. This completes the proof of the lemma.

Lemma 4.4.10. Let $A \in Y^{(r)}$ be regular, then for $\sigma(\mathcal{U}(A)), d(1)=d(2)=d(3)$.

Proof By symmetry, we only need to show $d(1)=d(2)$. Let $\mathcal{H}_{1}=\{F \in \sigma(\mathcal{U}(A)): 1 \in$ $F$ and $2 \notin F\}, \mathcal{H}_{2}=\{F \in \sigma(\mathcal{U}(A)): 1 \notin F$ and $2 \in F\}$.

Claim. If $F \in \mathcal{H}_{2}$, then $(F \backslash\{2\}) \cup\{1\} \in \mathcal{H}$.

Case $1.3 \notin F$

Let $G=Y \backslash F$, the $G \in \mathcal{U}_{3}(A)$. If $(F \backslash\{2\}) \cup\{1\} \notin \mathcal{H}_{1}$, then $F \backslash\{2\} \in \partial^{(k-1)} \mathcal{U}(A)$. It's easy to see that $\{5,4\} \subseteq F \backslash\{2\}$ (because $Y \backslash F \in \mathcal{U}_{3}(A)$ ), so $F \backslash\{2\} \in \partial^{(k-1)} \mathcal{U}_{0}(A)$. Therefore $\exists A_{0} \in \mathcal{U}_{0}(A)$ such that $G \cup A_{0}=[3, n]$, which is equivalent to $(F \backslash\{2\}) \cap\left(Y \backslash A_{0}\right)=\emptyset$. By crossintersecting property, $A \cup\left(\operatorname{conj}_{1}(A)+\{3\}\right)=[3, n]$. But $\min B(A)-1 \notin A$ and $\min B(A)-1 \notin$ $\operatorname{conj}_{1}(A)$. It's a contradiction.

Case 2. $3 \in F$

Let $G=Y \backslash F$, then $G \in \mathcal{U}_{0}(A)$. If $(F \backslash\{2\}) \cup\{1\} \in \mathcal{H}_{1}$, then $F \backslash\{2\} \in \partial^{(k-1)} \mathcal{U}(A)$. Because 
$2 \notin F \backslash\{2\}, 3 \in F \backslash\{2\}$, we know $F \backslash\{2\} \in \partial^{(k-1)} \mathcal{U}_{3}(A)$. This will lead to the same contradiction in Case 1.

This completes the proof of the claim.

Define a map $\tau: \mathcal{H} \rightarrow \mathcal{H}_{1}$ as $\tau(F)=(F \backslash\{2\}) \cup\{1\}$. Clearly the map is 1-1. Therefore $\left|\mathcal{H}_{2}\right| \leq\left|\mathcal{H}_{1}\right|$.

By symmetry, we can show that $\left|\mathcal{H}_{1}\right| \leq\left|\mathcal{H}_{2}\right|$. Thus $\left|\mathcal{H}_{1}\right|=\left|\mathcal{H}_{2}\right|$. It follows that $d(1)=$ $d(2)$.

The next lemma says that if $\sigma(\mathcal{A})$ satisfies the degree condition then the degrees of 2 and 3 in $\mathcal{A}$ cannot be too small.

Lemma 4.4.11 (degree principal). Let $A \in Y^{(r)}$ be regular and $\mathcal{A}=\mathcal{U}(A)$. For $s<|\mathcal{A}|$ let $\mathcal{A}^{*}=\mathcal{L}(\mathcal{A}, s)$.

(1) If $\mathcal{B} \subseteq \mathcal{A}$ then the element 1 has the maximum degree in $\sigma(\mathcal{B})$.

(2) If $\left|\mathcal{B}_{3}\right| \geq\left|\mathcal{A}_{3}^{*}\right|$ and $\left|\partial^{(k-1)} \mathcal{B}_{\overline{3}}\right| \leq\left|\partial^{(k-1)} \mathcal{A}_{3}^{*}\right|$ then the element 1 has the maximum degree in $\sigma(\mathcal{B})$.

(3) If $\left|\mathcal{B}_{2}\right| \geq\left|\mathcal{A}_{2}^{*}\right|$ and $\left|\partial^{(k-1)} \mathcal{B}_{\overline{2}}\right| \leq\left|\partial^{(k-1)} \mathcal{A}_{2}^{*}\right|$ then the element 1 has the maximum degree in $\sigma(\mathcal{B})$.

(4) If $\left|\mathcal{B}_{3}\right| \leq\left|\mathcal{A}_{3}^{*}\right|$ and $\left|\partial^{(k-1)} \mathcal{B}_{\overline{3}}\right| \geq\left|\partial^{(k-1)} \mathcal{A}_{\overline{3}}^{*}\right|$ and at least one of the inequalities is strict then the element 1 has the maximum degree in $\sigma(\mathcal{B})$.

(5) If $\left|\mathcal{B}_{2}\right| \leq\left|\mathcal{A}_{2}^{*}\right|$ and $\left|\partial^{(k-1)} \mathcal{B}_{\overline{2}}\right| \geq\left|\partial^{(k-1)} \mathcal{A}_{\overline{2}}^{*}\right|$ and at least one of the inequalities is strict then the element 1 has the maximum degree in $\sigma(\mathcal{B})$.

Proof (1) Let $\mathcal{F}=\sigma(\mathcal{B})$. It suffices to show that $\left|\mathcal{F}_{\overline{2}}\right| \geq\left|\mathcal{F}_{\overline{1}}\right|$ and $\left|\mathcal{F}_{\overline{3}}\right| \geq\left|\mathcal{F}_{\overline{1}}\right|$. By symmetry, we need only show $\left|\mathcal{F}_{\overline{2}}\right| \geq\left|\mathcal{F}_{\overline{1}}\right|$. First we observe that

$$
\mathcal{F}_{\overline{2}}=\left\{Y \backslash B: B \in \mathcal{B}_{2}\right\} \cup\left(Y^{(k-1)} \backslash \partial^{(k-1)} \mathcal{B}_{\overline{2}}+\{1\}\right)
$$

Therefore

$$
\left|\mathcal{F}_{\overline{2}}\right|=\left|\mathcal{B}_{2}\right|+\left(\begin{array}{l}
n-1 \\
k-1
\end{array}\right)-\left|\partial^{(k-1)} \mathcal{B}_{\overline{2}}\right|
$$

Let $\mathcal{C}=\mathcal{A} \backslash \mathcal{B}$. First we look at the case where $\mathcal{C} \subseteq \mathcal{A}_{2}$. Let $\mathcal{G}=\sigma(\mathcal{A})$. By Lemma 4.4.10, $\left|\mathcal{G}_{\overline{1}}\right|=\left|\mathcal{G}_{\overline{2}}\right|$. Clearly $\left|\mathcal{F}_{\overline{1}}\right|=\left|\mathcal{G}_{\overline{1}}\right|-|\mathcal{C}|$ and $\left|\mathcal{B}_{2}\right|=\left|\mathcal{A}_{2}\right|-|\mathcal{C}|$. It follows that $\left|\mathcal{F}_{\overline{2}}\right|=\left|\mathcal{F}_{\overline{1}}\right|$. 
If $\mathcal{C} \nsubseteq \mathcal{A}_{2}$, this case can be obtained from the previous case by replacing some sets in $\mathcal{C}$ with sets in $\mathcal{A}_{\overline{2}}$. So $\left|\mathcal{B}_{2}\right|$ will be increased and $\left|\partial^{(k-1)} \mathcal{B}_{\overline{2}}\right|$ will not decrease. It follows that $\left|\mathcal{F}_{\overline{2}}\right| \geq\left|\mathcal{F}_{\overline{1}}\right|$.

$(2),(3),(4)$ and (5) follow from the same argument in (1).

To describe the general procedure to take out some sets we need to introduce some definitions.

Definition 4.4.12. For $A \in Y^{(r)}$ define $N(A)$ as follows: let $A^{\prime}$ be the set obtained from $A$ by changing every element $x \in B(A)$ to $x-1$. If $A^{\prime}$ has only one block then define $N(A)$ to be the set obtained from $A$ by changing $\min B(A)$ to $\min B(A)-1$, otherwise define $N(A)=A^{\prime}$.

For example, $N(\{14,13,12,11,7,6,5,4\})=\{13,12,11,10,7,6,5,4\}$, $N(\{12,11,10,9,8,7,5,4\})=\{12,11,10,9,8,6,5,4\}$.

Definition 4.4.13. Let $A \in Y^{(r)}$ be regular. Define

$\mathcal{W}_{3}(A)=\left\{F \in \mathcal{U}_{3}(A): F \succ \operatorname{conj}_{1}(N(A))+\{3\}\right\}$,

$\mathcal{W}_{2}(A)=\left\{F \in \mathcal{U}_{2}(A): F \succ \operatorname{conj}_{1}(N(A))+\{2\}\right\}$,

$\mathcal{W}_{32}(A)=\left\{F \in \mathcal{U}_{32}(A): F \succ \operatorname{conj}_{1}(N(A))+\{3,2\}\right\}$,

$\mathcal{W}(A)=\mathcal{W}_{3}(A) \cup \mathcal{W}_{2}(A) \cup \mathcal{W}_{32}(A)$.

In the above example, $A=\{12,11,10,9,8,7,5,4\}, N(A)=\{12,11,10,9,8,6,5,4\}$, $\mathcal{W}_{3}(A)=[15,8] 73$, $\mathcal{W}_{2}(A)=[15,8] 72$ $\mathcal{W}_{32}(A)=[15,8] 732$.

Definition 4.4.14. Let $A \in Y^{(r)}$ be regular, $|\mathcal{U}(A) \backslash \mathcal{W}(A)|<s<|\mathcal{U}(A)|$, define $\gamma(A, s)=\mathcal{U}(A) \backslash \mathcal{W}(A) \cup \mathcal{L}(\preceq, \mathcal{W}(A), s-|\mathcal{U}(A) \backslash \mathcal{W}(A)|)$

Clearly, $|\gamma(A, s)|=s . \gamma(A, s)$ gives a way to take out $t$ sets from $\mathcal{U}(A)$ : take out the last $t$ sets of $\mathcal{W}(A)$ in the order " $\preceq$ ". The next theorem states that this is the optimal way to take out $t$ sets in order to minimize the shadow.

Theorem 4.4.15. Let $A \in Y^{(r)}$ be regular, $|\mathcal{U}(A) \backslash \mathcal{W}(A)|<s<|\mathcal{U}(A)|$. Let $\mathcal{B} \subseteq \mathcal{U}(A)$. If $\mathcal{U}_{0}(A)=\mathcal{V}_{0}(\mathcal{B})$ and $|\mathcal{B}|=s$, then $\left|\partial^{(l)} \gamma(A, s)\right| \leq\left|\partial^{(l)} \mathcal{B}\right|$. 
Proof We can assume that $\mathcal{V}_{0}(\mathcal{B}), \mathcal{V}_{3}(\mathcal{B}), \mathcal{V}_{2}(\mathcal{B})$ and $\mathcal{V}_{32}(\mathcal{B})$ are all initial segments in "£". Suppose $\gamma(A, s) \neq \mathcal{B}$, then without loss of generality we may assume $\mathcal{B}_{2} \supset\left(\mathcal{U}_{2}(A) \backslash \mathcal{W}_{2}(A)\right)$, $\mathcal{B}_{32} \subset\left(\mathcal{U}_{32}(A) \backslash \mathcal{W}_{32}(A)\right)$. Let $\mathcal{B}^{\prime}=\mathcal{R}\left(\mathcal{F}, \mathcal{U}_{2}(A), \mathcal{U}_{2}(A) \backslash \mathcal{W}_{2}(A), \mathcal{U}_{32}(A)\right.$. It can be verified that $\left|\partial^{(l)} \mathcal{B}^{\prime}\right| \leq\left|\partial^{(l)} \mathcal{B}\right|$ by Theorem 4.1.1. For other cases, we apply the same construction, and finally will get $\gamma($ A.s $)$.

Notice that $\mathcal{V}_{0}(\mathcal{A})$ is an initial segment in COLEX. Intuitively if we take out some sets from $\mathcal{V}_{3}(\mathcal{A}) \cup \mathcal{V}_{2}(\mathcal{A}) \cup \mathcal{V}_{32}(\mathcal{A})$ and add the same number of sets in $\mathcal{V}_{0}(\mathcal{A})$ we might reduce the size of the shadow.

Example $n=15, k=6, r=8, s=456$.

Here is a candidate:

$$
\begin{aligned}
& {[15,6]} \\
& {[15,6] 5} \\
& {[15,6] 4} \\
& {[15,7] 54} \\
& {[15,8] 654} \\
& {[15,8] 3} \\
& {[15,8] 2} \\
& {[15,8] 32}
\end{aligned}
$$

This candidate has size 469 . We need to take out 13 sets. Again we will take out the last 13 sets in $[15,8] 32$. The resulting set system is:

$[15,6]$

$[15,6] 5$

$[15,6] 4$

$[15,7] 54$

$[15,8] 654$

$[15,8] 3$

$[15,8] 2$

$[15,9] 32$

$[15,10] 832$

$[15,12] 9832$

$[15,13] 119832$ 
The size of the 5 -shadow of this set system is

$$
\left(\begin{array}{c}
10 \\
5
\end{array}\right)+\left(\begin{array}{c}
10 \\
4
\end{array}\right)+\left(\begin{array}{c}
10 \\
4
\end{array}\right)+\left(\begin{array}{l}
9 \\
3
\end{array}\right)+\left(\begin{array}{l}
8 \\
2
\end{array}\right)+\left(\begin{array}{l}
8 \\
4
\end{array}\right)+\left(\begin{array}{l}
8 \\
4
\end{array}\right)+\left(\begin{array}{l}
7 \\
3
\end{array}\right)+\left(\begin{array}{l}
6 \\
2
\end{array}\right)+\left(\begin{array}{l}
4 \\
1
\end{array}\right)+\left(\begin{array}{l}
3 \\
0
\end{array}\right)=979
$$

This is a better candidate.

Denote the first candidate by $\mathcal{D}_{1}$, the second by $\mathcal{D}_{2}$. Comparing $\mathcal{D}_{1}$ with $\mathcal{D}_{2}$, we can see that

$$
[15,8] 654 \subseteq \mathcal{D}_{2} \backslash \mathcal{D}_{1}, \quad[15,9] 83 \subseteq \mathcal{D}_{1} \backslash \mathcal{D}_{2} .
$$

Intuitively if we remove $[15,9] 83$ from $\mathcal{D}_{1}$ and add the same size of the initial segment of $[15,8] 654$ to it, then because $|\{6,5,4\}|>|\{8,3\}|$ and by Theorem 4.1.1, the size of the shadow will go down or stay the same. This explains why $\mathcal{D}_{2}$ is better than $\mathcal{D}_{1}$.

We can go further and make $\mathcal{V}_{0}(\mathcal{A})$ even larger. Here is a third candidate:

$$
\begin{aligned}
& {[15,6]} \\
& {[15,6] 5} \\
& {[15,6] 4} \\
& {[15,7] 54} \\
& {[15,8] 654} \\
& {[15,12] 7654} \\
& {[15,9] 3} \\
& {[15,10] 83} \\
& {[15,11] 983} \\
& {[15,12] 10983} \\
& {[15,9] 2} \\
& {[15,10] 82} \\
& {[15,11] 982} \\
& {[15,12] 10982} \\
& {[15,9] 32} \\
& {[15,10] 832} \\
& {[15,11] 9832} \\
& {[15,12] 109832}
\end{aligned}
$$

This candidate has size exactly 456 . We denote this set system by $\mathcal{D}_{3}$. The size of the 5 -shadow of this set system is 982 . It is not as good as $\mathcal{D}_{2}$. 
Comparing $\mathcal{D}_{2}$ with $\mathcal{D}_{3}$, we can see that

$$
[15,12] 7654 \subseteq \mathcal{D}_{3} \backslash \mathcal{D}_{2}, \quad[15,12] 1110983 \subseteq \mathcal{D}_{2} \backslash \mathcal{D}_{1}
$$

However, this time $|\{7,6,5,4\}|<|\{11,10,9,8,3\}|$. It is wiser to remove $[15,12] 7654$ from $\mathcal{D}_{3}$ and add the same size of the initial segment of $[15,12] 1110983$ to it.

From these candidates we can see that the structures of the last member in $\mathcal{V}_{0}(\mathcal{A})$ and its conjugate determines which candidate is better. So we have the following definition.

Definition 4.4.16. Let $A \in Y^{(r)}$ be regular. $A$ is said to be normal if $\forall x \in A$ with $6 \leq x \leq$ $\min B(A)$, we have $|A \cap[4, x-1]|>\left|\operatorname{conj}_{1}(A) \cap[4, x-1]\right|+1$.

In the above example, $\{12,11,10,9,8,7,5,4\}$ and $\{12,11,10,9,8,6,5,4\}$ are normal, while $\{15,14,13,12,7,6,5,4\}$ is not.

Proposition 4.4.17. If $A$ is normal, then so is $N(A)$.

Definition 4.4.18. Let $A \in Y^{(r)}$. For $i=1, \cdots$, len $(r, A)$, define $M_{i}(A)=\operatorname{mem}(P(r, A, i))$.

Now we are ready to answer the question. We can take $A^{*}$ to be the maximum set in $Y^{(r)}$ such that $A^{*}$ is normal and $\left|\mathcal{U}\left(A^{*}\right)\right| \geq s$, then take out some sets to make its size $s$ by letting $\mathcal{A}^{*}=\gamma(A, s)$. Then $\mathcal{A}^{*}$ has the minimum $(k-1)$-shadow.

Lemma 4.4.19. Let $n, k, s$ be such that $n>2 k$ and $c(3,2)<s \leq c(5,3)$. Let $r=n-k-1$ and let $A$ be the maximum (with respect to "£”) set in $Y^{(r)}$ such that $A$ is normal and $|\mathcal{U}(A)| \geq s$. Let $i$ be an integer such that $2 \leq i<\operatorname{len}(A)$. Let $\mathcal{A}^{\prime}=\mathcal{U}\left(M_{i}(A)\right), \mathcal{A}^{\prime \prime}=\mathcal{U}\left(M_{i+1}(A)\right)$. Let $\mathcal{A} \subseteq Y^{(r)}$ be a set system with $|\mathcal{A}|=s$ that satisfies:

(1) $\mathcal{V}_{0}(\mathcal{A}), \mathcal{V}_{3}(\mathcal{A}) \backslash\{3\}, \mathcal{V}_{2}(\mathcal{A}) \backslash\{2\}$ and $\mathcal{V}_{32}(\mathcal{A}) \backslash\{3,2\}$ are all initial segments in “ $\preceq$ ".

(2) $\mathcal{V}_{0}\left(\mathcal{A}^{\prime}\right) \subseteq \mathcal{V}_{0}(\mathcal{A}) \subseteq \mathcal{V}_{0}\left(\mathcal{A}^{\prime \prime}\right)$

(3) $\mathcal{V}_{3}(\mathcal{A}) \subseteq \mathcal{V}_{3}\left(\mathcal{A}^{\prime}\right), \mathcal{V}_{2}(\mathcal{A}) \subseteq \mathcal{V}_{2}\left(\mathcal{A}^{\prime}\right)$ and $\mathcal{V}_{32}(\mathcal{A}) \subseteq \mathcal{V}_{32}\left(\mathcal{A}^{\prime}\right)$

(4) either the element 1 has the maximum degree in $\sigma(\mathcal{A})$ or $\mathcal{A}^{\prime} \subseteq \mathcal{A}$.

Then there exists $\mathcal{B} \in Y^{(r)}$ with $|\mathcal{B}|=s$ that satisfies:

(a) $\mathcal{V}_{0}(\mathcal{B}), \mathcal{V}_{3}(\mathcal{B}) \backslash\{3\}, \mathcal{V}_{2}(\mathcal{B}) \backslash\{2\}$ and $\mathcal{V}_{32}(\mathcal{B}) \backslash\{3,2\}$ are all initial segments in "”.

(b) $\mathcal{V}_{0}(\mathcal{A}) \subseteq \mathcal{V}_{0}(\mathcal{B})$ 
(c) $\mathcal{V}_{3}(\mathcal{B}) \subseteq \mathcal{V}_{3}\left(\mathcal{A}^{\prime \prime}\right), \mathcal{V}_{2}(\mathcal{B}) \subseteq \mathcal{V}_{2}\left(\mathcal{A}^{\prime \prime}\right)$ and $\mathcal{V}_{32}(\mathcal{B}) \subseteq \mathcal{V}_{32}\left(\mathcal{A}^{\prime \prime}\right)$

(d) either the element 1 has the maximum degree in $\sigma(\mathcal{B})$ or $\mathcal{A}^{\prime \prime} \subseteq \mathcal{B}$.

(e) $\left|\partial^{(l)} \mathcal{B}\right| \leq\left|\partial^{(l)} \mathcal{A}\right|$.

Proof We will construct $\mathcal{B}$ from $\mathcal{A}$ via a series of transformations:

(i) If $\mathcal{V}_{32}(\mathcal{A}) \supset \mathcal{V}_{32}\left(\mathcal{A}^{\prime \prime}\right)$ and $\mathcal{V}_{2}(\mathcal{A}) \subset \mathcal{V}_{2}\left(\mathcal{A}^{\prime \prime}\right)$ then let $\mathcal{A}^{(1)}=\mathcal{R}\left(\mathcal{A}, \mathcal{V}_{2}(\mathcal{A}), \mathcal{V}_{2}\left(\mathcal{A}^{\prime \prime}\right), \mathcal{V}_{32}(\mathcal{A})\right)$, else let $\mathcal{A}^{(1)}=\mathcal{A}$. In other words, we fill in the missing part of $\mathcal{V}_{2}\left(\mathcal{A}^{\prime \prime}\right)$ with the extra sets in $\mathcal{V}_{32}(\mathcal{A})$. (ii) If $\mathcal{V}_{32}\left(\mathcal{A}^{(1)}\right) \supset \mathcal{V}_{32}\left(\mathcal{A}^{\prime \prime}\right)$ and $\mathcal{V}_{3}\left(\mathcal{A}^{(1)}\right) \subset \mathcal{V}_{3}\left(\mathcal{A}^{\prime \prime}\right)$ then let

$$
\mathcal{A}^{(2)}=\mathcal{R}\left(\mathcal{A}^{(1)}, \mathcal{V}_{3}\left(\mathcal{A}^{(1)}\right), \mathcal{V}_{3}\left(\mathcal{A}^{\prime \prime}\right), \mathcal{V}_{32}\left(\mathcal{A}^{(1)}\right),\right.
$$

else let $\mathcal{A}^{(2)}=\mathcal{A}^{(1)}$

(iii) If $\mathcal{V}_{32}\left(\mathcal{A}^{(2)}\right) \nsubseteq \mathcal{V}_{32}\left(\mathcal{A}^{\prime \prime}\right)$ then let $\mathcal{A}^{(3)}=\left(\mathcal{A}^{(2)} \backslash\left(\mathcal{K} \cup \mathcal{V}_{0}\left(\mathcal{A}^{(2)}\right)\right) \cup \mathcal{L}\left(Y^{(k)},\left|\mathcal{V}_{0}\left(\mathcal{A}^{(2)}\right)\right|+|\mathcal{K}|\right)\right.$ where $\mathcal{K}=\mathcal{V}_{32}\left(\mathcal{A}^{(2)}\right) \backslash \mathcal{V}_{32}\left(\mathcal{A}^{\prime \prime}\right)$, else let $\mathcal{A}^{(3)}=\mathcal{A}^{(2)}$. In other words, we remove the extra part of $\mathcal{V}_{32}\left(\mathcal{A}^{(2)}\right)$ that exceeds $\mathcal{V}_{32}\left(\mathcal{A}^{\prime \prime}\right)$ and add the same amount of sets to $\mathcal{V}_{0}\left(\mathcal{A}^{(2)}\right)$.

(iv) If $\mathcal{V}_{2}\left(\mathcal{A}^{(3)}\right) \nsubseteq \mathcal{V}_{2}\left(\mathcal{A}^{\prime \prime}\right)$ then let $\mathcal{A}^{(4)}=\left(\mathcal{A}^{(3)} \backslash\left(\mathcal{K} \cup \mathcal{V}_{0}\left(\mathcal{A}^{(3)}\right)\right)\right) \cup \mathcal{L}\left(Y^{(k)},\left|\mathcal{V}_{0}\left(\mathcal{A}^{(3)}\right)\right|+|\mathcal{K}|\right)$ where $\mathcal{K}=\mathcal{V}_{2}(\mathcal{A}) \backslash \mathcal{V}_{2}\left(\mathcal{A}^{\prime \prime}\right)$, else let $\mathcal{A}^{(4)}=\mathcal{A}^{(3)}$. (For simplicity, we use the symbol $\mathcal{K}$ again.)

(v) If $\mathcal{V}_{3}\left(\mathcal{A}^{(4)}\right) \nsubseteq \mathcal{V}_{3}\left(\mathcal{A}^{\prime \prime}\right)$ then let $\mathcal{B}=\left(\mathcal{A}^{(4)} \backslash\left(\mathcal{K} \cup \mathcal{V}_{0}\left(\mathcal{A}^{(4)}\right)\right)\right) \cup \mathcal{L}\left(Y^{(k)},\left|\mathcal{V}_{0}\left(\mathcal{A}^{(4)}\right)\right|+|\mathcal{K}|\right)$ where $\mathcal{K}=\mathcal{V}_{3}(\mathcal{A}) \backslash \mathcal{V}_{3}\left(\mathcal{A}^{\prime \prime}\right)$, else let $\mathcal{B}=\mathcal{A}^{(4)}$.

Obviously $|\mathcal{A}|=\left|\mathcal{A}^{(1)}\right|=\left|\mathcal{A}^{(2)}\right|=\left|\mathcal{A}^{(3)}\right|=\left|\mathcal{A}^{(4)}\right|=|\mathcal{B}|$. It's easy to see that $\left|\partial^{(l)} \mathcal{A}^{(2)}\right| \leq$ $\left|\partial^{(l)} \mathcal{A}^{(1)}\right| \leq\left|\partial^{(l)} \mathcal{A}\right|$. Since $A$ is normal, by the same argument in Theorem 4.1.1, we have $\left|\partial^{(l)} \mathcal{B}\right| \leq\left|\partial^{(l)} \mathcal{A}^{(4)}\right| \leq\left|\partial^{(l)} \mathcal{A}^{(3)}\right| \leq\left|\partial^{(l)} \mathcal{A}^{(2)}\right|$. It follows that $\left|\partial^{(l)} \mathcal{B}\right| \leq\left|\partial^{(l)} \mathcal{A}\right|$.

(a), (b) and (c) are all trivial from the construction of $\mathcal{B}$. It remains to verify (d). We distinguish two cases.

Case 1. $\mathcal{A}^{\prime} \subseteq \mathcal{A}$.

It follows immediately that $\mathcal{V}_{3}\left(\mathcal{A}^{\prime}\right) \subseteq \mathcal{V}_{3}(\mathcal{A})$. Because $\mathcal{V}_{3}\left(\mathcal{A}^{\prime \prime}\right) \subseteq \mathcal{V}_{3}\left(\mathcal{A}^{\prime}\right)$, by the construction of $\mathcal{B}, \mathcal{V}_{3}\left(\mathcal{A}^{\prime \prime}\right) \subseteq \mathcal{V}_{3}(\mathcal{B})$. By the same argument, $\mathcal{V}_{2}\left(\mathcal{A}^{\prime \prime}\right) \subseteq \mathcal{V}_{2}(\mathcal{B})$ and $\mathcal{V}_{32}\left(\mathcal{A}^{\prime \prime}\right) \subseteq \mathcal{V}_{32}(\mathcal{B})$. If $\mathcal{V}_{0}\left(\mathcal{A}^{\prime \prime}\right) \subseteq \mathcal{V}_{0}(\mathcal{B})$ then $\mathcal{A}^{\prime \prime} \subseteq \mathcal{B}$. If not, by the degree principal, the element 1 has the maximum degree in $\sigma(\mathcal{B})$.

Case 2. the element 1 has the maximum degree in $\sigma(\mathcal{A})$.

If $\mathcal{V}_{3}\left(\mathcal{A}^{\prime \prime}\right) \subseteq \mathcal{V}_{3}(\mathcal{B}), \mathcal{V}_{2}\left(\mathcal{A}^{\prime \prime}\right) \subseteq \mathcal{V}_{2}(\mathcal{B})$ and $\mathcal{V}_{32}\left(\mathcal{A}^{\prime \prime}\right) \subseteq \mathcal{V}_{32}(\mathcal{B})$ it becomes the situation in Case 1.

So we first assume $\mathcal{V}_{3}(\mathcal{B}) \subset \mathcal{V}_{3}\left(\mathcal{A}^{\prime \prime}\right)$. If $\mathcal{V}_{0}(\mathcal{B}) \subseteq \mathcal{V}_{0}\left(\mathcal{A}^{\prime \prime}\right)$ then by the degree principal, the element 1 has the maximum degree in $\sigma(\mathcal{B})$. If $\mathcal{V}_{0}\left(\mathcal{A}^{\prime \prime}\right) \subset \mathcal{V}_{0}(\mathcal{B})$, then $\mathcal{V}_{0}(\mathcal{A}) \subset \mathcal{V}_{0}(\mathcal{B})$. Thus we 
know transformation (iii) was not performed because (ii) would be performed before (iii). By the degree principal, the element 1 has the maximum degree in $\sigma(\mathcal{B})$.

If $\mathcal{V}_{2}(\mathcal{B}) \subset \mathcal{V}_{2}\left(\mathcal{A}^{\prime \prime}\right)$, the above argument will still hold. So last we assume $\mathcal{V}_{32}(\mathcal{B}) \subset \mathcal{V}_{32}\left(\mathcal{A}^{\prime \prime}\right)$ Clearly (i), (ii) and (iii) were not performed. Again, by the degree principal, the element 1 has the maximum degree in $\sigma(\mathcal{B})$.

Theorem 4.4.20. Let $n, k, s$ be such that $n>2 k$ and $c(3,2)<s \leq c(5,3)$. Let $r=n-k-1$ and let $A$ be the maximum (with respect to "”) set in $Y^{(r)}$ such that $A$ is normal and $|\mathcal{U}(A)| \geq s$. Let $\mathcal{A} \subseteq Y^{(r)}$ with $|\mathcal{A}|=s$. If the element 1 has the maximum degree in $\sigma(\mathcal{A})$, then $\left|\partial^{(l)} \gamma(A, s)\right| \leq$ $\left|\partial^{(l)} \mathcal{A}\right|$.

Proof By Theorem 4.3.13 in section 4.3, we may assume that $\left|\mathcal{V}_{0}(\mathcal{A})\right| \geq\left(\begin{array}{c}n-5 \\ r\end{array}\right)+2\left(\begin{array}{c}n-5 \\ r-1\end{array}\right)$. We can further assume that $\mathcal{V}_{0}(\mathcal{A}), \mathcal{V}_{3}(\mathcal{A}), \mathcal{V}_{2}(\mathcal{A})$ and $\mathcal{V}_{32}(\mathcal{A})$ are all initial segments in "”.

Case 1. $\max \mathcal{V}_{0}(\mathcal{A}) \preceq A$.

Let $i$ be the largest integer such that $M_{i}(A) \preceq \max \mathcal{V}_{0}(\mathcal{A})$. Let $\mathcal{A}^{\prime}=\mathcal{U}\left(M_{i}(A)\right), \mathcal{A}^{\prime \prime}=$ $\mathcal{U}\left(M_{i+1}(A)\right)$. By degree principal, $\mathcal{V}_{3}(\mathcal{A}) \subseteq \mathcal{V}_{3}\left(\mathcal{A}^{\prime}\right), \mathcal{V}_{2}(\mathcal{A}) \subseteq \mathcal{V}_{2}\left(\mathcal{A}^{\prime}\right)$ and $\mathcal{V}_{32}(\mathcal{A}) \subseteq \mathcal{V}_{32}\left(\mathcal{A}^{\prime}\right)$. So by Lemma 4.4.19, there exists $\mathcal{B}$ that satisfies the conclusions of lemma 4.4.19. If $|\mathcal{B}|<\left|\mathcal{A}^{\prime \prime}\right|$ then by Theorem 4.4.15 $\left|\partial^{(l)} \gamma\left(A^{\prime \prime}, s\right)\right| \leq\left|\partial^{(l)} \mathcal{B}\right|$. In this case, for convenience let $\mathcal{B}=\gamma\left(A^{\prime \prime}, s\right)$. Now repeat this proof for $M_{i+1}(A), M_{i+2}(A)$ and $\mathcal{B}$, and finally this will lead to the desired inequality.

Case 2. $\max \mathcal{V}_{0}(\mathcal{A}) \succ A$.

Let $A^{\prime}$ be the maximum member in $Y^{(r)}$ such that $A^{\prime} \succeq \max \mathcal{V}_{0}(\mathcal{A}), \operatorname{len}\left(A^{\prime}\right)=\operatorname{len}\left(\max \mathcal{V}_{0}(\mathcal{A})\right)$ and $\left|\mathcal{U}\left(A^{\prime}\right)\right| \geq s$. Let $A^{\prime \prime}=N\left(A^{\prime}\right)$. By the maximality of $A, A^{\prime}$ is not normal. Claim that $\left|\mathcal{U}\left(A^{\prime \prime}\right)\right|<s$, otherwise it will contradict the maximality of $A^{\prime}$. By a similar method in Theorem 4.4.15, we can find $\mathcal{B} \in Y^{(r)}$ such that $\left|\partial^{(l)} \mathcal{B}\right| \leq\left|\partial^{(l)} \mathcal{A}\right|$ and $\left(\mathcal{U}\left(A^{\prime \prime}\right) \backslash \mathcal{U}_{0}\left(A^{\prime \prime}\right)\right) \subseteq\left(\mathcal{B} \backslash \mathcal{V}_{0}(\mathcal{B})\right)$. Since $A^{\prime}$ is not normal, using the same method in the proof of Lemma 4.4.19, we can remove $\mathcal{V}_{0}(\mathcal{B}) \backslash \mathcal{U}_{0}(A)$ and add the same amount of sets to $\mathcal{U}_{3}(A) \backslash \mathcal{V}_{3}(\mathcal{B}), \mathcal{U}_{2}(A) \backslash \mathcal{V}_{2}(\mathcal{B})$ and $\mathcal{U}_{32}(A) \backslash \mathcal{V}_{32}(\mathcal{B})$. Denote the new set system by $\mathcal{B}^{\prime}$. So $\left|\partial^{(l)} \mathcal{B}^{\prime}\right| \leq\left|\partial^{(l)} \mathcal{B}\right|$. Clearly $\mathcal{B}^{\prime} \subseteq \mathcal{U}(A)$ since $|\mathcal{U}(A)| \geq s$. Therefore $\left|\partial^{(l)} \gamma(A, s)\right| \leq\left|\partial^{(l)} \mathcal{B}^{\prime}\right|$ by Theorem 4.4.15, which completes the proof of this Case.

Theorem 4.4.21. Let $n, k, s$ be such that $n>2 k$ and $c(3,2)<s \leq c(5,3)$. Let $r=n-k-1$ and let $A^{*}$ be the maximum (with respect to " $\preceq$ ") set in $Y^{(r)}$ such that $A^{*}$ is normal and $\left|\mathcal{U}\left(A^{*}\right)\right| \geq s$. Let $\mathcal{A}^{*}=\gamma\left(A^{*}, s\right), \mathcal{F}^{*}=\sigma\left(\mathcal{A}^{*}\right)$. Let $\mathcal{F} \subseteq X^{(k)}$ be an intersecting family with $c(\mathcal{F})=s$, then $|\mathcal{F}| \leq\left|\mathcal{F}^{*}\right|$. 
Proof Let $\mathcal{A}=\mathcal{F}_{\overline{1}}$. Since

$$
\mathcal{F} \subseteq\{Y \backslash A: A \in \mathcal{A}\} \cup\left(Y^{(k-1)} \backslash \partial^{(k-1)} \mathcal{A}+\{1\}\right),
$$

in view of Theorem 4.4.20, we have $|\mathcal{F}| \leq\left|\mathcal{F}^{*}\right|$. 


\section{Chapter 5}

\section{An Asymptotic Bound and}

\section{Non-existence Theorem}

\subsection{Introduction}

In this chapter we will prove some asymptotic results on intersecting set systems. To be more general, we will state and prove the theorems for $t$-intersecting set systems, which reduce to intersecting set systems in the special case $t=1$.

\subsection{Intersecting set systems with a fractional degree condition}

We first look at the problem of finding the maximum size of an intersecting set system $\mathcal{F} \subseteq X^{(k)}$ such $d(\mathcal{F}) \leq e|\mathcal{F}|$ for some real number $e \in(0,1)$. In a well known but unpublished paper, Erdös, Rothschild and Szemerédi showed that if $e=\frac{2}{3}$ then $|\mathcal{F}| \leq\left|\mathcal{F}_{(3,2)}\right|$ for fixed $k$ and $n$

sufficiently large, where $\mathcal{F}_{(3,2)}=\left\{F \in X^{(k)}:|F \cap\{1,2,3\}| \geq 2\right\}$. In this section we will sharpen this result.

We use the following definition from [5]. 
Definition 5.2.1 ([5]). Let $X=[n]$ and $\mathcal{F} \subseteq X^{(k)}$ be a t-intersecting set system. Let $\mathcal{F}^{(t)}=$ $\left\{G \subseteq X: \exists F \in \mathcal{F}\right.$ with $G \subseteq F$ and $\left.|G \cap F| \geq t \forall F^{\prime} \in \mathcal{F}\right\}$. Define the base $\mathcal{B}$ of $\mathcal{F}$ as:

$$
\mathcal{B}=\left\{B \in \mathcal{F}^{(t)}: \nexists F \in \mathcal{F}^{(t)}, F \subset B\right\}
$$

For $A \subseteq X$, the restriction of $\mathcal{F}$ on $A$ is defined as

$$
\mathcal{F} \mid A=\{H \subseteq A: \exists F \in \mathcal{F} \text { such that } F \cap A=H\}
$$

Lemma 5.2.2 ([5]). $|\mathcal{B}| \leq \phi(k)$ where $\phi$ is a function that depends only on $k$.

Lemma 5.2.3. Let $\mathcal{F} \subseteq X^{(k)}$ be a maximal t-intersecting set system, $X=[n]$. If $n>2 k$ then the base $\mathcal{B}$ of $\mathcal{F}$ is t-intersecting.

Proof Assume to the contrary that there exist $B_{1}, B_{2} \in \mathcal{B}$ with $\left|B_{1} \cap B_{2}\right|<t$. Since $\left|B_{i}\right|<k, i=$ 1,2 and $n>2 k$, we have $\left|X \backslash B_{2}\right|>k$. So we can find $F_{1} \in X^{(k)}$ with $B_{1} \subseteq F_{1} \subseteq\left(X \backslash B_{2}\right) \cup B_{1}$. Because $B_{1} \subseteq F_{1}$ and $B_{1} \in \mathcal{B}$, by the definition of the base, $\left|F \cap F_{1}\right| \geq t, \forall F \in \mathcal{F}$. Since $\mathcal{F}$ is maximal, $F_{1} \in \mathcal{F}$. But $\left|F_{1} \cap B_{2}\right|=\left|B_{1} \cap B_{2}\right|<t$, a contradiction to $B_{2} \in \mathcal{B}$.

Lemma 5.2.4. Let $\mathcal{F} \subseteq X^{(k)}$ be a t-intersecting set system, $X=[n]$. Let $Y \subseteq X$ be a minimum size subset of $X$ such that $\mathcal{G}=\mathcal{F} \mid Y$ is t-intersecting. Then $|\mathcal{G}| \leq f(k)$ where $f$ is a function that depends only on $k$.

Proof If $n \leq 2 k$ the lemma is trivial because $n$ is a function of $k$. So we may assume $n>2 k$. Extend $\mathcal{F}$ to a maximal $t$-intersecting set system $\mathcal{F}^{*}$. Let $\mathcal{B}^{*}$ be the base of $\mathcal{F}^{*}$. By Lemma 5.2.2, $\left|\mathcal{B}^{*}\right| \leq \phi(k)$. If we let $A=\cup_{B \in \mathcal{B}^{*}} B$, then $|A| \leq \varphi(k)$ for some function $\varphi$ that depends only on $k$. By Lemma 5.2.3, $\mathcal{B}^{*}$ is $t$-intersecting. Because every member $F \in \mathcal{F}^{*}$ contains some member $B \in \mathcal{B}^{*}, \mathcal{F}^{*} \mid A$ is $t$-intersecting. Since $\mathcal{F} \subseteq \mathcal{F}^{*}, \mathcal{F} \mid A$ is also $t$-intersecting. By the minimality of $Y,|Y| \leq|A|$. It follows that $|\mathcal{G}| \leq f(k)$.

Theorem 5.2.5. Let $\mathcal{F} \in X^{(k)}$ be a t-intersecting set system, $X=[n]$. Suppose for $c=\frac{t+1}{t+2}$, $d(\mathcal{F}) \leq c|\mathcal{F}|$. Then for $n>n_{0}(k),|\mathcal{F}| \leq(t+2)\left(\begin{array}{c}n-t-2 \\ k-t-1\end{array}\right)$ with "=" if and only if there exists $D \in X,|D|=t+2$, and $\mathcal{F}=\left\{F \in X^{(k)}:|F \cap D|=t+1\right\}$, denoted by $\mathcal{F}_{t+2, t+1}^{*}$.

Proof By way of contradiction, suppose $\mathcal{F}$ is maximal and $|\mathcal{F}|>(t+2)\left(\begin{array}{l}n-t-2 \\ k-t-1\end{array}\right)$. Let $Y$ be the minimum subset of $X$ such that $\mathcal{F} \mid Y$ is $t$-intersecting. Let $\mathcal{G}=\mathcal{F} \mid Y$. By Lemma 5.2.4, 
$|\mathcal{G}| \leq f(k)$. Clearly $|G| \geq t$ for every $G \in \mathcal{G}$, and if $|G|=t$ for some $G$ then $\mathcal{F}$ is trivial, contradicting the degree condition. So we may assume $|G| \geq t+1$ for each $G \in \mathcal{G}$. Let

$\mathcal{G}_{t+1}=\{G \in \mathcal{G}:|G|=t+1\}$. For $G \in \mathcal{G}$ with $|G|=l$, there are $\left(\begin{array}{c}n-|Y| \\ k-l\end{array}\right)$ ways to complete $G$ to $F \in \mathcal{F}$. So for large $n$, in order for $|\mathcal{F}|>(t+2)\left(\begin{array}{c}n-t-2 \\ k-t-1\end{array}\right)$, we must have $\left|\mathcal{G}_{t+1}\right| \geq t+2$.

Case 1. There exist distinct $G_{1}, G_{2}, G_{3} \in \mathcal{G}_{t+1}$ with $G_{1} \cap G_{2}=G_{1} \cap G_{3}$.

Let $D_{1}=G_{1} \cap G_{2}$, then $\left|D_{1}\right|=t, G_{1}=D_{1} \cup\left\{x_{1}\right\}, G_{2}=D_{1} \cup\left\{x_{2}\right\}, G_{3}=D_{1} \cup\left\{x_{3}\right\}$, with $x_{1}, x_{2}, x_{3}$ all distinct. We claim that $G \supseteq D_{1}$ for every $G \in \mathcal{G}_{t+1}$. If not, $\left|G \cap D_{1}\right| \leq t-1$. But $G$ must $t$-intersect $G_{1}, G_{2}, G_{3}$, so $\left\{x_{1}, x_{2}, x_{3}\right\} \subseteq G$, and $\left|G \cap D_{1}\right| \geq t-1$. Therefore $|G| \geq\left|D_{1}\right|+\left|\left\{x_{1}, x_{2}, x_{3}\right\}\right|=t-1+3=t+2$, a contradiction. This proves the claim. Now $\mathcal{G}_{t+1}$ is trivial. Pick $i \in D_{1}$. Let $\mathcal{H}=\{F \in \mathcal{F}: i \notin \mathcal{F}\}$. Then $|\mathcal{H}|<\left(\begin{array}{l}n-t-2 \\ k-t-1\end{array}\right)$ for large $n$. But

$\frac{|\mathcal{F}|-|\mathcal{H}|}{|\mathcal{F}|}<c$. So $|\mathcal{F}|<\left(\begin{array}{c}n-t-2 \\ k-t-1\end{array}\right)$ for $n>n_{0}(k)$, a contradiction.

Case 2. There do not exist 3 members in $\mathcal{G}_{t+1}$ as described in Case 1.

Pick $G_{1}, G_{2} \in \mathcal{G}_{t+1}$. Let $D_{1}=G_{1} \cap G_{2}, D=G_{1} \cup G_{2}$. Then $\left|D_{1}\right|=t,|D|=t+2, G_{1}=D_{1} \cup\left\{x_{1}\right\}$, $G_{2}=D_{1} \cup\left\{x_{2}\right\}$. For every $G \in \mathcal{G}_{t+1}$, by assumption, $\left|G \cap D_{1}\right| \leq t-1$. But $G$ must $t$-intersect $G_{1}$ and $G_{2}$, so $\left\{x_{1}, x_{2}\right\} \subseteq G$, and $\left|G \cap D_{1}\right|=t-1$. Therefore $|G \cap D|=t+1$. Since $\left|\mathcal{G}_{t+1}\right| \geq t+2$, all the $t+2(t+1)$-subsets of $D$ must be present in $\mathcal{G}_{t+1}$. Now for every $G \in \mathcal{G}$ such that $|G \cap D| \leq t$ we can find $G^{\prime} \in \mathcal{G}_{t+1}$ satisfying $\left|G \cap G^{\prime}\right| \leq t-1$, hence $|G \cap D| \geq t+1, \forall G \in \mathcal{G}$. By the minimality of $Y$, we have $Y=D$. We claim that $D \notin \mathcal{G}$. If not, let $\mathcal{G}=\left\{G_{1}, G_{2} \cdots, G_{t+2}\right\}$. So $\mathcal{G}=\mathcal{G}_{t+1} \cup\{D\}$. Let $G_{0}=D$. Let $\mathcal{F}_{i}=\left\{F \in \mathcal{F}: F \cap D=G_{i}\right\}, i=0,1, \cdots, t+2$. So $\mathcal{F}_{0}, \mathcal{F}_{1}, \cdots, \mathcal{F}_{t+2}$ is a partition of $\mathcal{F}$. Let $f_{i}=\left|\mathcal{F}_{i}\right|, s=f_{1}+f_{2}+\cdots+f_{t+2}$. By the structure of $\mathcal{G}_{t+1}$, because every point appears in $G_{0}=D$, the sum of every $t+1$ numbers out of $f_{1}, f_{2}, \cdots, f_{t+2}$ is no more than $\frac{t+1}{t+2} s$. Therefore $f_{1}=f_{2}=\cdots=f_{t+2}$. If $D \in \mathcal{G}$, the degree condition will be violated. This proves the claim. Now $\mathcal{G}=\{G \subseteq D:|G|=t+1\}$. It follows that $|\mathcal{F}| \leq(t+2)\left(\begin{array}{c}n-t-2 \\ k-t-1\end{array}\right)$. Clearly "=" holds if and only if $\mathcal{F} \cong \mathcal{F}_{t+2, t+1}^{*}$.

The special case $t=1$ is the following corollary.

Corollary 5.2.6. Let $\mathcal{F} \in X^{(k)}$ be an intersecting set system. If $d(\mathcal{F}) \leq \frac{2}{3}|\mathcal{F}|$, then for $n>$ $n_{0}(k),|\mathcal{F}| \leq\left|\mathcal{F}_{(3,2)}\right|$ with "=" if and only if $\mathcal{F}$ is isomorphic to $\mathcal{F}_{(3,2)}$, where $\mathcal{F}_{(3,2)}^{*}=\{F \in$ $\left.X^{(k)}:|F \cap\{1,2,3\}|=2\right\}$. 


\subsection{Minimum complementary degree of an intersecting set sys- tem}

In this section we turn to the minimum complementary degree of intersecting set systems. We will look at the problem of how large can the minimum complementary degree be for an intersecting set system and let $c(i)=|\mathcal{F}|-d(i), c(\mathcal{F})=\min _{i \in X} c(i)$.

Theorem 5.3.1. Let $\mathcal{F} \subseteq X^{(k)}$ be a t-intersecting set system, $|X|=n$. Then for $n>n_{0}(k)$, $c(\mathcal{F}) \leq\left(\begin{array}{c}n-t-2 \\ k-t-1\end{array}\right)$.

Proof Let $\mathcal{G}$ be defined as before.

(1) If $\mathcal{G}_{t} \neq \emptyset$, then there exists $G \in \mathcal{G}_{t}$, so by definition of $\mathcal{G}$, every $F \in \mathcal{F}$ contains $G$, thus $c(\mathcal{F})=0$. So assume $\mathcal{G}_{t}=\emptyset$.

(2) If $\mathcal{G}_{t+1}=\emptyset$, since there are $\left(\begin{array}{c}n-l \\ k-l\end{array}\right)$ ways to extend an $l$-set to $F \in \mathcal{F}, c(\mathcal{F}) \leq|\mathcal{F}| \leq$ $f(k)\left(\begin{array}{l}n-t-2 \\ k-t-2\end{array}\right) \leq\left(\begin{array}{l}n-t-2 \\ k-t-1\end{array}\right)$ for some function $f(k)$. The first inequality is obvious, the second follows from Lemma 5.2.4, and the third holds for $n>n_{o}(k)$.

(3) If $\left|\mathcal{G}_{t+1}\right|=1$, let $G_{1} \in \mathcal{G}_{t+1}$. Pick $i \in G_{1}$. Then every $G \in \mathcal{G}$ that does not contain $i$ must have cardinality at least $t+2$. The same argument as in (2) works.

(4) Let $G_{1}, G_{2} \in \mathcal{G}_{t+1}$, then $\left|G_{1} \cap G_{2}\right|=t,\left|G_{1} \cup G_{2}\right|=t+2$. Pick $i \in G_{1} \cap G_{2}$. Let $F \in \mathcal{F}$. In order for $F$ to $t$-intersect both $G_{1}$ and $G_{2}, F$ must contain $G_{1} \cup G_{2} \backslash\{i\}$. But $\left|G_{1} \cup G_{2} \backslash\{i\}\right|=t+1$. By the same argument as in $(2), c(i) \leq\left(\begin{array}{l}n-t-2 \\ k-t-1\end{array}\right)$ for $n>n_{0}(k)$.

The special case $t=1$ is the following corollary.

Corollary 5.3.2. Let $\mathcal{F} \in X^{(k)}$ be an intersecting set system. Then when $n>n_{0}(k), c(\mathcal{F}) \leq$ $\left(\begin{array}{l}n-3 \\ k-2\end{array}\right)$. 


\section{Bibliography}

[1] B. Bollobás, On generalized graphs, Acta Math. Acad. Sci. Hungar. 16(1965) 447-452.

[2] B. Bollobás, Combinatorics, Cambridge University Press, 1986.

[3] P. Erdős, C. Ko, R.Rado, Intersection theorems for systems of finite sets, Quarterly J. of Mathematics, Oxford 12(1961), 313-320.

[4] P. Frankl, Generalizations of theorems of Katona and Milner, Acta Math. Acad, Scient. Hung. 25(1976), 359-363.

[5] P. Frankl, On intersecting families of finite sets, Journal of Combinatorial Theory, Series A 24, 146-161 (1978).

[6] P. Frankl, Erdős-Ko-Rado theorem with conditions on the maximal degree, J. Combinatorial Th A 46(1987), 252-263.

[7] P. Frankl and Z. Füredi, A short proof for a theorem of Harper about Hamming spheres, Discrete Math 34(1981), 311-313.

[8] P. Frankl and N. Tokushige, Some best possible inequalities concerning cross-intersecting families, J. Comb. Th. A 61(1992) 87-97.

[9] J. Goldwasser, Erdős-Ko-Rado with conditions on the minimum complementary degree, $J$. Comb. Theory, Ser. A 109(1): 45-62 (2005).

[10] J. Goldwasser and Y. Ou, Maximum size of $t$-cross-intersecting set systems, submitted.

[11] L.H. Harper, Optimal numberings and isoperimetric problems on graphs, J. Combinatorial Th 1(1966), 385-394.

[12] A.J.W. Hilton, The Erdös-Ko-Rado theorem with valency conditions, unpublished manuscript, 1976. 
[13] G.O.H. Katona, Intersection theorems for systems of finite sets, Acta Math. Acad. Sci. Hung. 15(1964), 329-337.

[14] G.O.H. Katona, A simple proof of the Erdős-Ko-Rado theorem, J. Comb. Math. B 13(1972) 183-184.

[15] G.O.H. Katona, A simple proof of a theorem of Milner, J. Comb. Th. A 83(1998) 138-140.

[16] G.O.H. Katona, A theorem of finite sets, in Theory of Graphs, Proc. Coll. Tihany 1966, Akad. Kiado Budapest (1968), 187-20\%.

[17] G.O.H. Katona, private commucation, 2003.

[18] A. Kisvölcsey, exact bounds on cross-intersecting families, Graphs and Combinatorics 17(2001), 275-287.

[19] D.J. Kleitman, On a combinatorial conjecture of Erdős, J. Combinatorial Theory 1(1966), 209-214.

[20] D.J. Kleitman, On a conjecture of Milner on $k$-graphs with non-disjoint edges, J. combinatorial Theory 5(1968), 153-156.

[21] J.B. Kruskal, The number of simplicies in a complex, in Mathematical Optimization Techniques, Univ. Calif. Press (1963), 251-278.

[22] D. Lubell, A short proof of Sperner's lemma, J, Comb. Th. 1(1966) 299.

[23] M. Matsumoto and N. Tokushige, The exact bound in the Erdős-Ko-Rado theorem for cross-intersecting families, J. Comb. Th. A 52(1989) 90-97.

[24] M. Matsumoto and N. Tokushige, A generalization of the Katona theorem for cross $t$ intersecting families, Graphs and Combinatorics 5(1989) 159-171.

[25] L. Meshalkin, A generalization of Sperner's theorem on the number of subsets of a finite set, Theory of Probab. and its Applic. 8(1964) 204-205.

[26] E. Milner, A combinatorial theorem on systems of sets, J. London math Society 43(1968) 204-206.

[27] L. Pyber, A new generalization of the Erdős-Ko-Rado theorem, J. Comb. Th. A 43(1986) $85-90$.

[28] K. Yamamoto, Logarithmic order of free distributive lattices, J. Math. Soc. Japan 6(1954) 343-353. 